\title{
Zur Classification der Flächen dritter Ordnung.
}

\section{Von}

Carr. Rodenberg in Plauen ïm Vogtlande.

(Mit 9 lithographirten Tafeln.)

Die Eintheilung der Flächen dritter Ordnung hinsichtlich der Realität ihrer Geraden und der möglichen singulären Punkte wurde erschöpfend gegeben von Herrn Schläfli in seiner grossen Arbeit: On the Distribution of Surfaces of the third order into Species, in reference to the absence or presence of Singular Points. Philos. transact. London 1863 , p. 207 ff.

Gelegentlich meiner Promotion gab Clebseh mir die Untersuchung des Pentaeders für die einzelnen Schläfli'schen Arten, welche Aufgabe in meiner Dissertation: „Das Pentaeder der Flächen dritter Ordnung beim Auftreten von Singularitäten" behandelt wird. Hierbei stellte es sich heraus, dass eine Reihe specieller Flächen, insbesondere diejenigen mit conischen Knoten, noch ein eigentliches Pentaeder besitzen, während andere, wie die mit biplanaren Knoten, im Allgemeinen Pentaeder mit unendlich benachbarten Ebenen mit sich führen. Aber nicht auf alle denkbaren Fälle der vereinigten Lage wird man unter Zugrundelegung eiver bestimmten Singularität geführt. Diese Thatsache veranlasste mich, die umgekehrte, und wohl zweckmässigere, Fragestellung nach denjenigen Flächen, welche einem gegebenen Pentaeder angehören, zu wählen. Die Behandlung der hieraus entspringenden Aufgabe ist in diesem Aufsatze durchgeführt.

Von der grössten Wichtigkeit für die folgenden Untersuchungen ist der von Herrn $\mathrm{Kl}$ ein in seiner Abhandlung "Ueber Flächen dritter Ordnung“, Math. Annalen Bd. VI, p. 551 fi., gegebene Satz, dass alle Flächen ohne Singularitäten und derselben Schlätli'schen Art darch continuirliche Aenderung der Constanten in einander übergeführt werden können, ohne dass hierbei ein Knoten auftrete. Diese Derivation ist nämlich fast ausnahmslos auch dann noch möglich, wenn das Pentaeder unverändert bestehen bleibt. 
Die trennenden Flächen der verschiedenen Arten besitzen einen conischen Knoten und zwar lassen sich aus diesen Flächen die allgemeinen in ausserordentlich einfacher Weise mit Hülfe eines Verfahrens gewinnen, das wir gleich betrachten werden.

Gerade so, wie der Kegel zweiter Ordnung den Uebergang von einem einschaligen Hyperboloid $z u$ einem zweischaligen bildet, kann man eine Fläche mit Knoten als Uebergang zwischen zwei andern ansehen, bei denen sich die Theile in der Nähe des Knotens verhalten wie die angeführten Flächen zweiter Ordnung. Bezeichnet man die beiden Processe, denen ein Knoten hiernach unterworfen werden kann, durch "Verbinden $(+)^{\prime \prime}$ und „Trennen $(-)^{*}$, so erhält man nach Herrn Klein alle Flächen ohne Singularitäten, wenn man an jedem Knoten einer Fläche mit vieren einen jener Processe anbringt, nämlich:

$$
\begin{aligned}
& ++++\cdots \text { I mit } 27 \text { reellen Geraden, } \\
& +++\cdots \text { II , } 15, " \text {, } \\
& ++\ldots \ldots \text { III }, 7, n \\
& +-\ldots \ldots \text { IV }, 3 \text {, } 3 \text { und } 7 \text { reellen Ebenen, } \\
& \text { - }-\ldots V \text { V } 3 \text { " } 3 \text { " "13 " }
\end{aligned}
$$

Die Flächen, bei denen Knoten bestehen bleiben, seien durch die Ziffer der Flächen ohne Knoten bezeichnet, welche aus jenen durch Verbinden hervorgehen. Rechnet man überhaupt alle Flächen zu einer Art, welche sich in einander üherführen lassen, ohne dass hierbei ein newer oder höherer singulärer Punlot auftritt, so genijgt jetzt die Ziffer nicht mehr zur völligen Bestimniung, da die Aenderung eines Knotens durch die biplanare Form zu einer neuen Art führen kann, welche sich in ihrem Zusammenhange nicht von der frühern unterscheidet.

Es ist nun klar, dass man leicht die Art, zu der eine gegebene Fläche dritter Ordnung gehört, angeben kann, wenn es gelingt, die gewissermassen mechanisch definirten Processe, + und - , algebraisch zu vollziehen. Dieses ist einfach, wenn, was stets geschehen soll, die Gleicbung der Fläche in ihrer kanonischen Form als Aggregat von $\check{5}$ Cuben rorausgesetzt wird und das Pentaeder erhalten bleibt. So lange die Ebenen desselben nicht an besondere Bedingungen geknüpft sind, ist bekanntlich die kanonische Form eindeutig bestimmt*). Gehen jedoch vier derselben durch einen Punkt, so sind diese selbst in gewisser Weise willkürlich und die Gleichung kann unendlich viele Formen annehmen $\left.{ }^{* *}\right)$.

*) Vergl. Clebsch, Deber die Knotenpunkte der Hesse'schen Fläche etc. Borchardt's Jonrnal Bd. 59 , p. $143 \mathrm{ff}$, auch Gordan Math. Ann. Bd. V, p. 34I ff.

**) Vergl. Eckardt: Ueber diejenigen Flächen, auf welchen sich drei gerade Linien in einem Punkte schneiden. Programm der Realschule zu Chemnitz 1875; oder Math. Ann. Bd. X, p. $227 \mathrm{ff}$. 
Eine andere Specialisirung geht hervor, wenn zwei oder mehrere Ebenen unendlich nahe liegen. Die Reduction auf fünf Cuben ist dann nicht mehr möglich, aber gewisse Gleichungsformen werden wir aufstellen, welche der allgemeinen völlig entsprechen. Treten nun auch in den abzuleitendeu kanonischen Formen noch andere willkürliche Elemente neben denen des Pentaeders auf, so sind doch diese im Allgemeinen völlig bestimmt.

Im Zusammenhange kaun nach dem Gesagten natürlich nur die Flächenschaar betrachtet werden, deren Glieder dasselbe Pentaeder haben. Damit haben aber dann gleichzeitig alle Flächen ihre Erledigung gefunden, deren Pentaeder eine gleiche Anzahl reeller Ebenen besitzen und auch hinsichtlich der vereinigten Lage einer beliebigen Anzahl von Ebenen übereinstimmen, da solche Pentaeder alle durch reelle Collineationen in einander überführbar sind.

Für Flächen mit eigentlichem Pentaeder ist es von grossem Vortheil, die Ebenen desselben direct als Coordinatenebenen zu wählen, da vamentlich die Discriminante der Fläche, deren Verschwinden das Anftreten ejnes Knotens andeutet, dann eine sehr einfache Form annimmt").

Im $\S 1$. schicken wir einige Sätze über ein derartiges Coordinatensystem voraus und wenden uns dann (\$§ 2.,4., う., 6.) za den ihm angehörigen Flächen; immer ausgehend von einer möglichst einfachen Form, aus der wir durch allmähliche Aenderung der Coefficienten alle Arten erzeugen. Hierbei kann jedoch nie biplanaren Knoten begegnet werden, des speciellen Pentaeders wegen, welche dieselben mit sich führen. Wir sind daher gezwungen, Durchgänge durch solche Punkte besonders zu behandeln ( $\$ 3$.$) .$

\$ 7. giebt, als Abschluss der Flächen mit eigentlichem Pentaeder, eine Vertheilung der Knoten aller Flächen, welche demselben angehören, auf den Raum, und die Aufzählung der getrennten Mannigfaltigkeiten, welche die einzelnen Arten constituiren.

§ 8. enthält einen Satz, mit dessen Hülfe sich in einfacher Weise (\$ 9.) die Gleichungen alier Flachen mit mehrfachen Pentaederebenen angeben lassen. Diese finden einzeln ihre Behandlung in den $\$ \$ 10 .-15$.

Die Flächen wit unbestimmtem Pentaeder sind in $\$ 16$. aufgezählt; untersucht in den $\$ \S 17 .-20$.

§ 21. enthält die tabellarische Zusammenstellung aller Resultate.

*) Vergl. Salmon: On Quarternary Cubics. Phil. Tr. 1860; p. 229 ff. 


\section{$\S 1$.}

Ueber ein Pentaedercoordinatensystem.

Unter Zugrundelegung von 5 Ebenen als Fundamentalebenen eines Coordinatensystems kann man definiren:

Die Coordinaten eines Punktes sind 5 Zahlen,

$$
\begin{array}{lllll}
x_{1} & x_{2} & x_{3} & x_{4} & x_{5}
\end{array}
$$

welche sich verhalten wie seine Abstände von den Ebenen des Pentacders, jeder Abstand multiplicirt mit einer so gewö̈hlten Constanten, dass die Summe der Producte:

wird.

$$
x_{1}+x_{2}+x_{3}+x_{1}+x_{5}=0
$$

Bei reellen Ebenen wird der Raum durch sie in $2^{4}-1=15$ Kammern zerlegt, entsprechend der Anzahl von Variationen von je vieren der Vorzeichen der Coordinaten eines Punkts weniger eins. Denn das Vorzeichen der fünften Coordinate kann man jederzeit fest annehmen und eine Variation kommt in Wegfall, da der Identität $\Sigma x_{i}=0$ wegen nicht alle $x$ gleiches Vorzeichen haben kö»nen.

Hinsichtlich der Vertheilung der Vorzeichen liegen zwei Möglichkeiten vor, entweder sind 1) vier Zeichen gleich, oder 2) nur drei gleich, die beiden übrigen diesen eutgegengesetzt. Im ersten Falle kann die fünfte Coordinate nie Null werden, denn es müsste sonst anch die Summe der übrigen verschwinden, was unmöglich ist. Folglich wird der zugehörige Raum nur durch 4 Ebenen begrenzt. Solcher giebt es hiernach $\mathbf{5}$, sie mögen Tetraederkammern genannt werden. Die übrigen 10 erfordern alle fünf Ebenen zu ihrer Begrenzung; sie mögen Pentaederkammern heissen.

Haben vier Coordinaten eines Punkts dasselbe Zeichen, so liegt er in einer Tetraederkammer, im andern Falle in einer Pentaederkammer.

Sind zwei Ebenen conjugirt imaginär, so wird durch die drei reellen der Raum noch in vier Kammern zerlegt, von denen diejenige ausgezeichnet ist, welche nicht von der, stets reellen, Schuittlinie der conjugirten Ebenen durchsetzt wird. Nennen wir die Ebenen

$$
x_{1}=0, \quad x_{2}=0, \quad x_{3}=0, \quad x_{4}+i x_{5}=0, \quad x_{4}-i x_{5}=0,
$$

so haben, wie unter Anwendung ähnlicher Betrachtungen, wie vorhin, leicht erwiesen wird, $x_{1} x_{2} x_{3}$ dasselbe Vorzeichen, wenn der Puntit in der Kammer liegt, welehe nicht von der isolirten Kante durchsetzt wird.

Sind endlich zwei Paare conjugirter Ebenen vorhanden, so tritt beine Theilung des Raumes mehr ein, denn eine reelle Ebene ist hierzu nicht mehr ausreichend.

Von Interesse sind für uns noch die 10 Ebenen, welche man durch die Schnittpunkte von je dreien der Fundamentalebenen und die Schnittr 
linie der beiden übrigen legen kann und die passend als Diagonalebenen zu bezeichnen sind. Solche Ebenen sind dargestellt durch die Gleichungen

$$
x_{1}+x_{2}=0 \text { etc. }
$$

Denn der angefủhrten genügt die Gerade $x_{1}=x_{2}=0$, aber auch in Folge der Identität - der Punkt $x_{3}=x_{4}=x_{5}=0$, wie verlangt wird.

Sehen wir ab von denjenigen Durchschnittserzeugnissen dieser Ebenen, welche in denen des Pentaeders völlig enthalten sind, so bleiben uns noch $10 \cdot 3=30$ Linien, welche zu dreien durch eine Pentaederecke gehen und von drei solchen Diagonalebenen erzeugt werden, deren Kanten sich in jener Ecke treffen. Dann giebt es noch $5 \cdot 4=20$ Schnittpunkte, von denen je vier die Spitzen der Dächer sind, welche von dreien solcher 6 Diagonalebenen, deren Kanten einer Tetraederkammer angehören, über deren Seitenflächen gebildet werden.

Von Vortheil für die Anschauung ist es, das Tetraeder regulär, die fünfte Ebene unendlich fern au denken. Dann halbiren die 6 erwähnten Diagonalebenen die Aussenwinkel des Tetraeders nud die 4 Ecken liegen auf den in den Mitten seiner Seitenflächen errichteten Normalen.

Mit Rücksicht auf die Gleichungen der Diagonalebenen kann man leicht angeben, wann ein Punlit in einer solchen Ebene oder in einer. der Schnittlinien oder einem der Schnittpunlte liegt: Es müssen resp. $2,3,4$ Coordinaten in ihren absoluten Werthen übereinstimmen und zwei dersellen mit entgegengesetzten Vorzeichen behaftet sein. Folglich können auch die übrigen wicht alle gleiches Zeichen haben und die Punkte gehören daher sämmtlich Pentaederkammern an, wie es die Alischaung auch ergiebt.

Ein theilweise imaginäres Pentaeder bedingt natürlich auch eine Anzahl imaginärer Diagonalebenen, dere山 Aufzählung ohne Schwierigkeit ist und füglich unterbleiben kann.

$\S 2$.

Die Flächen mit reellem Pentaeder.

Eine solche Fläche hat unter Zugrundelegung des erläuterten Systems die Gleichung

oder kurz

$$
\frac{x_{1}^{3}}{\alpha_{1}{ }^{2}}+\frac{x_{2}{ }^{3}}{\alpha_{2}{ }^{2}}+\frac{x_{3}{ }^{3}}{\alpha_{3}{ }^{2}}+\frac{x_{4}{ }^{3}}{\alpha_{4}{ }^{2}}+\frac{x_{5}{ }^{3}}{\alpha_{5}{ }^{2}}=0
$$

$$
\left(\alpha_{1}, \alpha_{2}, \alpha_{3}, \alpha_{4}, \alpha_{3}\right)
$$

Die gebrauchte Coefficientenbezeichnung wird sich als zweckmässig erweisen. Sollten einige Coefficienten negativ sein, so wären die ent-
sprechenden $\alpha$ imaginär zu nehmen. 
Ist die vorliegende Fläche eine specielle mit conischem Knoten y, so genügt dieser den Gleichungen

$$
\frac{y_{1}^{2}}{\alpha_{1}^{2}}=\frac{y_{2}^{2}}{\alpha_{2}^{2}}=\frac{y_{3}^{2}}{\alpha_{3}^{2}}=\frac{y_{4}^{2}}{\alpha_{4}^{2}}=\frac{y_{5}^{2}}{\alpha_{3}^{2}},
$$

d. h. die Grössen a sind dann geradezu die Coordinaten des Knotens und die Gleichung

$$
\alpha_{1}+\alpha_{2}+\alpha_{3}+\alpha_{4}+\alpha_{5}=0
$$

ist die Bedingung für sëin Auftreten, die Summe selbst die Discriminante der Fläche.

Hiernach ist jedem Punkte des Raumes eine Fläche dritter Ordnung mit Knoten zuertheilt und so die Mannigfaltigkeit von drei Dimensionen dieser Flächen, welche einem Pentaeder angehört, eindeutig. auf den Punktraum abgebildet*). Dieser kann demnach als Grenze des Bildes der Mannigfaltigkeit aller Flächen, deren jede durch 4 völlig willkürliche $\alpha$ gegeben ist, angesehen werden.

Nach den Entwickelungen am Schlusse des \& 1. findet man weiter, dass die Flächen mit 2, 3,4 Knoten sich abbilden resp. auf die Diagonalebenen, ihre Schnittinien und Schnittpunkte. Da nämlich die absoluten Werthe der Coordinaten als Quadratwurzeln der $\alpha^{2}$ völlig gegeben sind, so kann die Verschiedenheit nur in den Vorzeichen liegen, was wiederum mit der stets verschwindenden Summe nur im Einklange steht, wenn resp. 2, 3, 4 Coordinaten, ihrem absoluten Werthe nach, einander gleichkommen, woraus unsere Behauptung entspringt.

Es erscheint wünschenswerth, vor der Classification der Flächen an einigen besonders hierzu geeigneten Specialfällen die Lageuverhältnisse zwischen Fläche und Pentaeder kennen zu lernen und namentlich die Vertheilung der verschiedenen Arten von Knoten festzustellen.

Betrachten wir die Fläche mit der Maximalzahl der Knoten: 4. Nach dem eben. Gesagten besitzt deren Gleichung vier gleiche $\alpha^{2}$, deren Wurzeln - die Coordinaten der Knoten - sich mit der fünften auf vier verschiedene Weisen zur Summe Null combiniren lassen müssen. Nehmen wir die vier ersten Coefficienten $\frac{1}{\alpha^{2}}$ der Einbeit gleich, so ist die fünfte $\frac{1}{2^{2}}$, und die Gleichung der Fläche ist

$$
x_{1}^{2}+x_{2}^{2}+x_{3}^{2}+x_{4}^{2-}+\frac{x_{3}^{2}}{4}=0 \text {. }
$$

Die Knoten sind:

*) Eine Ausnahme bilden die Punkte der Pentaederebenen, welchen Ausartungen der Flächen in die dreifachen Pentaederebenen entsprechen, vergl. \$7., wo die Bedeutung dieser Ebenen als trennende Gebiete der verschiedenen Arten angegeben ist. 


$$
\begin{gathered}
-1,+1,+1,+1,-2, \\
1,-1,+1,+1,-2, \\
1,+1,-1,+1-2, \\
1,+1,+1,-1,-2 .
\end{gathered}
$$

Sie bilden die Ecken der kleinen Dächer, welche von Diagonalebeuen über den Ebenen des Tetraeders $x_{1}, x_{2}, x_{3}, x_{4}$ gebildet werden ( $\$ 1$.), und bestimmen ein Tetraeder, dessen Kanten diejenigen des andern schneiden.

Stellt man die Fläche so - wie es auf Tafel I der Klein'schen Arbeit geschehen ist -, dass eine Spitze des Knotenpunkttetraeders nach unten zeigt, so zeigt eine Spitze desjenigen der 4 Pentaederebenen nach oben und der Körper selbst erscheint um $60^{\circ}$ gegen den andern um die verticale Axe gedreht. Die fünfte Ebene liegt vereinigt mit der Ebene der einfachen Geraden.

Durch eine Kleine Aenderung der Coefficienten bönnen wir jetzt an beliebig vielen Knoten einen der Processe des Verbindeus und Trennens anbringen, wodurch alle Arten, welche direct aus unserer Specialfläche hervorgehen, abgeleitet sind. Die etwa bleibenden Knoten haben nun ersichtlich reelle Tangentenkegel, und man wird vermuthen, alle Knoten, welche wie sie in Pentaederkammern liegen, besitzen solche Kegel. Zur Erledigung dieser Frage betrachten wir die Gleichung des Tangentenkegels in dem Knoten $y$ :

$$
\frac{x_{1}^{2}}{y_{1}}+\frac{x_{2}^{2}}{y_{2}}+\frac{x_{3}^{2}}{y_{3}}+\frac{x_{1}^{2}}{y_{4}}+\frac{x_{5}^{2}}{y_{5}}=0 \text {. }
$$

Ein Kegel ist dann und nur dann reell, wenn er von einer willkïrlichen reellen Ebene in einer reellen Curve getroffen wird. Bei dem unserigen sind aber die Schnittcurven mit den Pentaederebenen reell, sobald drei, und nicht mehr, der $y$ dasselbe Zeichen haben, d. h. der Knoten die angegebene Lage hat. Die Schnittcurve und mit ihr der Kegel ist aber sicher imaginär, wenn vier der $y$ gleiches Zeichen haben, welches sich am besten für die fünfte Ebene als Schneidende ersehen lässt. Ein solcher Punkt liegt aber nach $\$ 1$. in einer Tetraederkammer. Folglich: $D_{i e}$ isolirten Knoten erfüllen die Tetraederkammern, die nicht isolirten die Pentaederliammern.

Nach diesen Erörterungen gehen wir behufs Begründung eirer Eintheilung von einer wlïche mit 27 reellen Geraden aus und führen diese durch allmähliche Aenderung der Coefficienten in andere Arten über, wobei wir die tremnenden Gebiete mit einem Knoten zu passiren
baben.

Zum Ausgange diene die Diagonalfläche von Clebsch

$$
(1,1,1,1,1) \text {. }
$$


Haben wir nun aus dieser nach vorgenommener Deformation eine Fläche mit Knoten erhalten, so verschwindet das Aggregat der $\alpha$ für eine bestimmte Vorzeichenvariation und wird nach dem bei fortgesetztem Deformiren erfolgenden Trennen der Flächentheile einen dem ursprünglichen im Zeichen entgegengesetzten Werth annehmeu, und so fort beim Passiren von weitern Knoten. Hieraus ziehen wir die für das Folgende fundamentale Regel: $O m$ zu erkennen, wie oft bei der Ueberführung einer Fläche mit 27 Graden in eine gegebene ein Knoten auftritt, bilde man bei beiden die Werthe der Aggregate der fün a für sämmtliche Vorzeichenvariationen - oder kurz die sämmtlichen Variationen -, die Anzahl derjenigen, welche einen Zeichenwechsel aufzuweisen haben, ist gleich der gesuchten.

Nimmt man insbesondere die Aenderung so vor, dass die einmal erzeugten Kroten erhalten bleiben, so kann man in der nämlichen Weise Flächen mit beliebig vielen derselben bis zur Maximalzahl 4 entstehen lassen. Nothwendig hat man aber nur bei der Ueberführung zweier Flächen mit gleich vielen Knoten in einander solche mit einem Knoten mehr als die vorliegenden zu passiren, wie aus den Abbildungen sofort hervorgeht.

Jedoch auch biplanare Knoten trennen unter Umständen die Gebiete der Flächen mit zwei cónischen. Solche können unter den unserigen aber nicht vorkommen, weil sie äusserst specielle Pentaeder besitzen*); sie machen sich überhaupt nur dann nothwendig, wenn man von einer Fläche mit 4 Knoten ausgeht, wie Herr Klein es thut.

Um bequem das Auftreten der Zeichenwechsel studiren zu können, ordnen wir die Coefficienten der Fläche

so, dass

$$
\begin{gathered}
\left(\alpha_{1}, \alpha_{2}, \alpha_{3}, \alpha_{4}, \alpha_{5}\right) \\
\alpha_{1}{ }^{2} \leqq \alpha_{2}{ }^{2} \leqq \alpha_{3}{ }^{2} \leqq \alpha_{4}{ }^{2} \leq \alpha_{5}{ }^{2} .
\end{gathered}
$$

Da ferner nur Verhältnisse in Betracht kommen, sei das grösste, $\alpha_{5}$, mit einem festen, dem negativen Zeichen belegt, wie es bei der Fläche mit 4 Knoten bereits geschehen ist. Für die Diagonalfäche haben wir dann die sämmtlichen Variationen in dem folgenden Schema, in dem das Zeichen von $\alpha_{5}$ gleich fortgelassen ist.
A

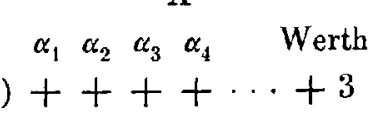
B
$\begin{array}{ccccc}\alpha_{1} & \alpha_{2} & \alpha_{3} & \alpha_{4} \quad \text { Werth }\end{array}$
2) $-+++\cdots+1$
3) $+-++\cdots+1$
4) $++-+\cdots+1$
5) $+++-\cdots+1$

*) Vergl. § 11. 

C
$\alpha_{1} \alpha_{2} \quad \alpha_{3} \quad \alpha_{1} \quad$ Werth
6) $--++\cdots-1$
7) $-+-+\cdots-1$
8) $-++\cdots \cdots-1$
9) $+--+\cdots-1$
10) $+-+\cdots \cdot-1$
11) $++\ldots-\cdots-1$
D

Es wirft sich zunächst die Frage nach der Maximalzahl der Zeichenwechsel auf, welche eine gegebene Fläche gegenüber der Diagonalfläche zeigen kann.

$Z u$ deren Beantwortung beweisen wir folgende 4 Hülfssätze:

1) Unter D tritt nie ein Zejchenwechsel auf.

2) Unter C tritt höchstens ein Zeichenwechsel auf und dann sind unter B zwei, soust virgends welche vorhanden.

3) Unter B können alle Variationen Zeichenwechsel haben, ist jedoch hier keiner vorhanden, so tritt überhaupt keiner auf.

4) Zeigt die Variation unter A einen Zeichenwechsel, so ist dasselbe mit allen unter B und mit keiner weitern der Fall.

Hieraus ergiebt sich als gesuchte Maximalzahl 5.

Die Richtigkeit der Sätze 1) und 4) leuchtet ohne Weiteres ein.

Zum Beweise von 2) bemerken wir, dass die zu betrachtende Variation jedenfalls 6 ) ist, denn diese ist die grösste. Also sei:

$$
-\alpha_{1}-\alpha_{2}+\alpha_{13}+\alpha_{1}-\alpha_{3}>0 \text {. }
$$

Bei der Annahme, es sei auch die nächst grösste 7)

$$
-\alpha_{1}+\alpha_{2}-\alpha_{3}+\alpha_{1}-\alpha_{3}>0
$$

erhalten wir als Summe beider Ungleichungen

d. h.

$$
-2 \alpha_{1}+2 \alpha_{1}-2 \alpha_{5}>0
$$

$$
a_{1}>a_{1}+\alpha_{5}
$$

was mit der gewählten Reihenfolge der $\alpha$ unvereinbar ist. Keinen Wechsel zeigen offenbar 1), 2), 3). Aus den Bildungen

$$
\begin{aligned}
& 5)+6)=2 \alpha_{3}-2 \alpha_{5}<0, \\
& 4)+6)=2 \alpha_{4}-2 a_{5}<0
\end{aligned}
$$

geht aber hervor, dass bei 4) und 5 ) Zeichenwechsel auftreten, womit unser Satz bewiesen ist.

Die erste Behauptung des Satzes 3) ergiebt sich, wenn man nur $a_{5}$ gross genug annimint.

Die zweite folgt daraus, dass für

$$
\text { 6) }>0
$$


auch

$$
\text { 6) }+5)=2 \alpha_{3}-2 \alpha_{s}>0
$$

welches unmöglich ist.

\section{Damit wären alle Hülfssätze erwiesen.}

Bei der Deformation der Diagonalfläche tritt unter B offenbar zuerst ein Verschwinden der Variation 5) - sagen wir eine Null auf, denn diese ist die kleinste und sonst ist nach dem Satze 3) noch keine aufgetreten. Aendern wir die Coefficienten weiter, doch so, dass die Null erhalten bleibt, so wird sich die zweite bei 4) einstellen. Von nun an können wir. jedoch in zwei vou einander wesentlich verschiedenen Richtungen Aenderungen vornehmeנ: Es können 1) zceitere Nullen unter $\mathrm{B}$ hervorgebracht werden; wir erhalten die Fläcke mit 4 Knoten; 2) Kann die mögliche Null unter $(\mathrm{C}$ erzeugt werden; wir crhalten eine Fläche mit 3 Knoten, welchen aber hein vierter (nach 2)) beitreten kann.

Die letztere Fläche ist nun eine solche, bei der sich Knoten durch die biplanare Form geändert baben, sofern sie aus einer mit 4 Knoten entstanden, denn nur solche setzen dem Auftreten der weitern Knoten Hindernisse entgegen.

Ihre Knoten zeigen aber, wie aus ihrer Gleichung

$$
(p, q, p+q, p+q, p+q)
$$

hervorgeht, im Verhalten nichts Verschiedenartiges, es ist kein Merkmal vorhanden, an wie vielen derselben der eben bezeichnete Process ausgefïhrt worden ist, wir finden nur eine Art. Herr Klein führt deren drei an, entsprechend der Inwerdung dieses Processes auf. 1, 2 , 3 Kroten und bezeiclnet überhaupt alle durch denselben aus irgend einer vorgelegteu Fläche entstandenen als neue Arten, während man unter Umständen nichts Neues erhält, wie wir sogrleich sehen werden. Der Schwerpunt der Sache liegt in jener Arbeit aber wohl darin, zu zeigen, dass nach Auflösung der Knoten besagte Aenderung überhaupt wirkungslos war*).

Bei Flächen mit einem, resp. zwei Knoten und lauter reellen Geraden haben wir von jeder nur eine Art. Erst wenn wir die Null unter $C$ hervorrufen und eine oder zwei der Nullen unter B in Zeichenwechsel überfübren, $d . h$. Flächentheile trennen, erhaltew wir andere Arten, als sie direct aus der Fläche mit 4 Knoten durch Trenuen erzeugt werden können.

Also nur bei flächen I mit drei Knoten, II mit zwei Knoten und III mit einem Knoten erhalten wir bis jetzt verschiedene Arten.

Ein noch möglicher Zeichenwechsel unter C liefert eine Fläche IV

*) Vgil. \$ 4. am Ende. 
ohne Knoten, welche aber von uns nur mit Hülfe des Durchgangs durch Knoten, an denen die Aenderung durch die biplanare Form angebracht ist, in eine Diagonalläche übergeführt werden kann.

Eine zweite Fläche von dieser Eigenschaft leiten wir aus der noch nicht betrachteten mit einem isolirten Knoten $a b$. Letztere erhält Herr Klein durch Ueberführung des Knotens einer IV durch die biplanare Form. Wir erhalten dieselbe unter Anwendung des Trennens auf alle Knoten einer Fläche mit vieren (oder algebraisch: durch Hervorrufen sämmtlicher Zeichenwechsel unter B) und schliessliches Znsammenziehen des kugelartigen Stücks der entspringenden $V$ zum Knoten, indem wir die Full unter A auftreten lassen. Aus der Gleichung der gefundenen Fläche:

$$
\left(\alpha_{1}, \alpha_{2}, \alpha_{3}, \alpha_{4}, \alpha_{1}+\alpha_{2}+\alpha_{3}+\alpha_{4}\right)
$$

ergeben sich als Coordinaten des Knotens:

$$
\alpha_{1}, \alpha_{2}, \alpha_{3}, \alpha_{4},-\left(\alpha_{1}+\alpha_{2}+\alpha_{3}+\alpha_{4}\right) .
$$

Derselbe liegt daher in einer Tetraederkammer und ist nach dem Frühern also wirklich isolirt, übereinstimmend mit dem soeben gewonnenen Resultate.

Aus dieser leiten wir nun die Fqäche

$$
\left(\alpha_{1}, \alpha_{2}, \alpha_{3}, \alpha_{4}, \alpha_{5}\right) \cdots \alpha_{5}>\alpha_{1}+\alpha_{2}+\alpha_{3}+\alpha_{4}
$$

ab. Der Knoten ist verschwunden, die Fläche eine IV, von der aus wir aber erst zu jener mit isolirtem Knoten zurückkehren müssen, um nach und nach Linien reell werden lassen zu können*).

Als Ergebniss wäre daher hinzustellen:

Bei allen Flächen ohne Singularitäten oder mit conischen Knoten kann man die Ueberführung ohne Ueberschreitung neuer Knoten auch dann noch vornehmen, wenn das Pentaeder bleibt, sofern sie nicht der Art IV ohne Knoten angehören. Letatere sind jedoch mit jener Beschränkung in 3 Unterarten zu spalten**). -

Es wird jetzt nothwendig, dass wir auf kurze Zeit unsere bisherigen Untersuchungen unterbrechen und Aenderungen durch biplanare Knoten wirklich ausführen, um namentlich die Frage nach der Wirkung dieses Vorgangs zu erörtern, wenn eine beliebige Anzahl vorhandener Knoten

*) Dass man wirklich eine Flüche mit 3 Geraden und 7 Ebenen vor sich bat, erkennt man auch durch ihre Ueberleitung in die Fläche

$$
\left(\alpha_{1}, a_{2}, \alpha_{3}, \alpha_{4}, \infty\right) \text {, }
$$

diese gehört der angegebenen Art an, wie Herr Eckardt a. a. 0. \$ 29. bewiesen hat, ein Knoten tritt nicht auf, folglich ist die unserige derselben Art.

**) Die Möglichkeit der Ueberführung in einander erhellt auch für diese Unterarten aus den $\$ \$ 12$. und 13. 
von ihm betroffen wird, da wir erst dann den Artbegriff exact zu begrenzen im Stande sein werden.

Dabei müssen wir ein gänzlich anderes Verfabren anwenden.

$\S 3$.

Die Durchgänge durch biplanare Knoten.

Wir wählen als Ausgangsfläche eine mit drei conischen Knoten, welche man stets durch die Gleichung

$$
\left.x_{1}^{3}+x_{1}{ }^{2}\left(x_{2}+x_{3}+x_{4}\right)+a x_{2} x_{3} x_{4}^{*}\right)
$$

darstellen kann. Die drei Knoten sind die in $x_{1}=0$ liegenden Ecken des Coordinatentetraeders. Die Flächenschaar, welche durch continuirliche Aenderung des $a$ erzeugt wird, enthält jedoch nicht unsere Uebergangsflächen, daher wählen wir die form

$$
x_{1}^{3}+x_{1}^{2}\left(\kappa x_{2}+\lambda x_{3}+\mu x_{4}\right)+x_{2} x_{3} x_{4}=0,
$$

welche uns gestattet, durch Ueberfübrung beliebig vieler der Grössen $x, \lambda, \mu$ durch Null zu Werthen mit entgegengesetzten Zeichen, an eben so vielen Knoten den fraglichen Process zu vollziehen; denn durch Nullsetzen mehrerer jener Grössen erhalten wir klächen mit biplanaren Knoten.

Man erkennt zunächst leicht (etwa durch Bildung der Discriminante der Schnittcurven unserer Fläche mit den Ebenen des Büschels $x_{1}+p x_{2}=0$ ), dass sich

$$
\text { eine }\left\{\begin{array}{l}
\text { I mit drei Knoten, } \\
\text { I mit vier Knoten, je nachdem } 1+2 \times \lambda \mu \geq_{0} \\
\text { II mit drei Knoten, }
\end{array}\right.
$$

findet. Hiermit stimmt überein, dass kurz vor dem Auftreten eines biplanaren Knotens die Fläche immer eine I ist**).

Wir zeigen nun, dass nur dann etwas Neues entsteht, wenn sich ein oder drei Knoten durch die biplanare Form ändern, dass es aber gleichgültig ist, welcher dieser beiden Fälle stattgefunden hat.

Zum Beweise betrachten wir die beiden tilächen:

$$
\begin{aligned}
& x_{1}{ }^{3}+x_{1}{ }^{2}\left(-x x_{2}+\lambda x_{3}+\mu x_{4}\right)+x_{2} x_{3} x_{4}=0, \\
& x_{1}{ }^{3}+x_{1}{ }^{2}\left(+x x_{2}+\lambda x_{3}+\mu x_{4}\right)+x_{2} x_{3} x_{4}=0,
\end{aligned}
$$

unter $x, \lambda, \mu$ positive Grössen verstanden. Diese beiden Flächen sind nicht anders als durch $x=0$ in einander überzuführen; in diesem Momente ist der Knoten 0, 1, 0, 0 biplanar, die Aenderung durch einen solchen Punkt ist ron Einfluss. Nehmen wir dieselbe jetzt bei einem zweiten Punkte, etwa $0,0,1,0$ vor, so entsteht die Flāche

*) Vergl. Scbläfli a. a. 0 .

*) Klein a. a. $0 . \S 5$. 


$$
x_{1}^{3}+x_{1}^{2}\left(\mathrm{x} x_{1}-\lambda x_{3}+\mu x_{4}\right)+x_{2} x_{3} x_{4}=0,
$$

welche durch die Transformation

$$
\begin{aligned}
& x_{1}=x_{1}^{\prime}, \\
& x_{2}=-x_{2}^{\prime}, \\
& x_{3}=-x_{3}^{\prime}, \\
& x_{1}=x_{1}^{\prime}
\end{aligned}
$$

in A übergeht. Eine an dritten Knoten angebrachte Aenderung führt daher wieder auf die Art B, da alle Knoten gleichwerthig sind, womit uusere Behauptung bewiesen ist.

Die Flächen mit weniger Knoten sind jetzt leicht erledigt.

Nach dem vorigen Paragraphen sind die in Frage stehenden Aenderungen auf solche Flächen ïberhaupt wirkungslos, welche aus den eben betrachteten durch Verbinden hervorgehen. Trennt man jedoch Flächentheile, so erzengt nur der Durchgang durch die biplanare Form bei einem der bleibenden Kuoten eine neue Art, denu derselbe, bei zweien augebracht, war ja einflusslos auf die ursprüngliche Fläche mit dreien.

Mit Rücksicht auf die bezüglich der Flächen mit einem Knoten bereits gewonnenen Resultate erhält man bei der Ueberfithrung conischer Knoten durch die. Ziplanare Form nur clan eine neue Flächenurt, wenn der Zusammenihang der Fläche nicht grösser als zu diesem Vorgang unbedingt nothwendig ist und eine ungerade Anzahl von Knoten von demselben betroffen wird. Man erbält ferner zwei klächen obne Knoten aus den beiden so abgeleiteten mit einem, - Flächen, wie sie aus der mit vier Knoten entstehen, als gegeben vorausgesetct -, welche sich bei festem Pentaeder nur durch die nämlichen Flächen in andere wit mehr reellen Linien überführen lassen, wenn man am Knoteu den Process des Trennens aubringt, sofern sein Kegel reell, oder ihn verschwinden lässt, sofern dieser Kegel imaginär ist. Alle hierher gehürigen Flächen mögen im Gegensatz zu denen, welche aus der nit vier Kuoten direct hervorsehen, als inverse Flächen bezeichnet werden.

Indem wir uns der Bezeichnung der Klein'schen Arbeit anschliessen und diese Arten durch angefügte Striche unterscheiden, haben wir als inverse Flächen die folgenden 6:

a) Flächen nit Knoten:

I' mit drei Knoten,

II' mit zwei Kuoten,

III' mit einem Ḱnoten,

IV' mit isolirtem Knoten,

b) Flächen ohue Knoteu:

IV' entstehend aus der gleichbenannten unter $a$ durch Verschwinden des Knotens,

IV" entstehend durch Auwendung des Trenuens aus der III'. 


\section{§ 4.}

\section{Die Flächen mit reellem Pentaeder. (Schlnss.)}

Es erübrigt noch die Flächen zu betrachten, in deren kanonischen Formen negative Coefficienten vorkommen. Von solchen haben wir nur diejenigen mit einem oder zwei derselben zu untersuchen. Deun wären mebr negativ, so könnten wir alle Zeichen entgegengesetzt nehmen und kämen auf eine der angeführten.

Um zu Flächen mit einem negativen Coefficienten zu gelangen, gehen wir aus von der IV'

$$
\left(\alpha_{1}, \alpha_{2}, \alpha_{3}, \alpha_{1}, \alpha_{5}\right) \cdots \alpha_{5}>\alpha_{1}+\alpha_{2}+\alpha_{3}+\alpha_{1}
$$

und führen $\alpha_{\mathrm{j}}$ durch $\infty$ ins Imaginäre. Dann jst

$$
\left(\alpha_{1}^{*}, \alpha_{2}, \alpha_{3}, \alpha_{1}, i \alpha_{5}\right)
$$

die gewünschte; eine I. $V^{\prime}$, da kein Knoten aufgetreten ist*). Die Summe der Quadratwurzeln ist jetzt complex nie Null: Eine Fläche mit einem negativen Coefficienten besitzt nie einen Knoten.

Lässt man nun in ähnlicher Weise einen zweiten Coefficienten negativ werden, so tritt so lange nichts Bemerkenswerthes ein, als nicht durch gegenseitiges Aufhebeu der imaginären Grössen, begleitet von geeigneten reellen, Veranlassung zu zwei imaginären Knoten gegeben wird. In diesem Falle rücken zwei der Geraden des reellen Dreiecks in die Verbindungslinie der Knoten - Axe bei Schläfli genamnt wir haben eine Schläfli'sche IV, 5; denu alle andern Flächen mit imaginären Knoten sind richt vom 'Zusanmenhange der IV'. Der Durchgang durch die eben betrachtete Fläche hat aber an der Gleichung gar kein Merkmal hinterlassen: Flächen mit imaginären Knoten bilden nie Uebergänge zu neuen Arten, wie das bekannt ist**).

Bemerkenswerth erscheint, dass Flächen mit reellem Pentaeder nie gleichzeitig reelle und conjugirte Knoten aufweisen, während alle übrigen Arten vertreten sind.

Der bessern Uebersicht wegen geben wir noch eine tabellarische Zusammenstellung und bringen hierbei in Erinnerung, dass inverse Flächen durch angehäugte Striche in der Bezeichnung gekenuzeichnet sind.

Fläche: $\left(\alpha_{1}, \alpha_{2}, \alpha_{3}, \alpha_{1}, \alpha_{5}\right) \cdots \alpha_{1}^{2} \leq \alpha_{4}^{2} \leq \alpha_{3}^{2} \leq \alpha_{1}^{2} \leq \alpha_{5}^{2} ; \alpha_{5}<0$

*) Vergl. eine Note des $\S 2$.

**) Vergl. z. B. Sturm: Synthetische Untersuchungen über Flächen dritter Ordnung, Cap. 8. Auch Klein a. a. O. \$ 7. Hier gind die Bilder uncerer Flächen die isolirten Doppelcurven. 
Variationen der Diagonalfiäche $(1,1,1,1,1)$

A

B

C

$$
\begin{aligned}
& \begin{array}{llll}
\alpha_{1} & \alpha_{2} & \alpha_{3} & \alpha_{4}
\end{array} \\
& \begin{array}{llll}
\alpha_{1} & \alpha_{2} & \alpha_{3} & \alpha_{1}
\end{array} \\
& \begin{array}{llll}
\alpha_{1} & \alpha_{2} & \alpha_{3} & \alpha_{4}
\end{array} \\
& ++++\cdots+ \\
& -+++\cdots+ \\
& --++\cdots \\
& +-++\cdots+ \\
& ++-+\cdots+ \\
& +t+\cdots+
\end{aligned}
$$

a) Flächen mit positiven Coefficienten.

1) Flächen ohne Knoten.

Anzah! der Zeichenwechsel: . Art:

0

1 unter B

$2 \#$ B

$3 ” B$

$2 \%$ B, 1 unter $\mathrm{C}$

$4 ”$ B

4 " B, 1 unter $\mathrm{A}$
I 27 reelle Linien,

II $15 \%$, , III $7 " * n$ IV $3 " \quad$ 7 reelle Eibenen, IV" 3 " " 7 $\mathrm{V} 3 \%$ " $13 \%$ IV' $3 "$ "

2) Flächen mit einem Knoten.

Anzahl der Zeichenwechsel:

0

1 unter B

$2 "$ B

$2 "$ B

$3 川$ B

$4 "$ B
Null unter:

B

B

B

C

B

A
Art:

I

II

III

III $^{\prime}$

IV

IV'

3) Flächen mit 2 Knoten.

Anzabl der Zeichenwecbsel:

0

1 unter $B$

$1 》 \mathrm{~B}$

$2 \%$ B
Nullen:

2 unter B

2 , B

1 B, 1 unter C

$2 "$ B
Art:

I

II

II' $^{\prime}$

III

4) Flächen mit 3 Knoten.

Anzahl der Zeichenwechsel:

0

0

1 unter B

Nullen:

Art:

3 unter B

2 , B, 1 unter C

$3 \% \mathrm{~B}$

I

$I^{\prime}$

II

5) Flächen mit 4 Knoten. 
b) Flächen mit negativen Coefficienten.

1 Coefficient negativ, IV'

2 Coefficienten negativ, IV' (oder Schläfli'sche IV, 5 mit zwei conjugirten Knoten).

Beispiel:

$$
\begin{aligned}
& x_{1}{ }^{3}+\frac{x_{2}{ }^{3}}{4}+\frac{x_{3}{ }^{3}}{9}+\frac{x_{4}{ }^{3}}{16}+\frac{x_{5}{ }^{3}}{16}=0 . \\
& (1,2,3,4,4) \\
& \text { B } \\
& \text { C } \\
& ++++\cdots+6-+++\cdots+4-\cdots++\cdots 0 \\
& +-++\cdots+2 \\
& ++-+\cdots 0 \\
& +++\cdots \cdot 2
\end{aligned}
$$

Also: Ein Zeichenwechsel unter B, eine Null unter B und eine Null unter C. Die Fläche ist eine $\mathrm{II}^{\prime}$ mit 2 Knoten.

\section{$\S 5$.}

Die Flächen mit zwei conjugirten Pentaederebenen.

Solche haben bekanntlich nie 27 reelle Linien, sondern, wie wir sehen werden, höchstens 15 .

Als Ausgangstäche zur Ableitung aller Arten wird sich irgend eine mit dem Maximum reeller Geraden am besten eignen, da vou diesen nur eine Art existirt.

Seien in der allgemeinen Gleichung unserer Flächen

$$
\frac{x_{1}{ }^{3}}{\alpha_{1}{ }^{2}}+\frac{x_{2}{ }^{3}}{\alpha_{2}{ }^{2}}+\frac{x_{3}{ }^{3}}{\alpha_{3}{ }^{2}}+\frac{\left(x_{4}+i x_{5}\right)^{3}}{\left(\alpha_{4}+i \alpha_{5}\right)^{2}}+\frac{\left(x_{4}-i x_{5}\right)^{3}}{\left(\alpha_{4}-i \alpha_{5}\right)^{2}}=0 \cdots \alpha_{1}{ }^{2} \leq \alpha_{2}{ }^{2} \leqq \alpha_{3}{ }^{2}
$$

oder

$$
\left(\alpha_{1}, \alpha_{2}, \alpha_{3}, \alpha_{4}+i \alpha_{5}, \alpha_{4}-i \alpha_{5}\right)
$$

vorläufig die Coefficienten der reellen Ebenen positiv, was offenbar zum Auftreten reeller Knoten nothwendig ist.

Von Diagonalebenen sind nur reell

$$
\begin{gathered}
x_{1}+x_{2}=0, \quad x_{2}+x_{3}=0, \quad x_{3}+x_{1}=0, \\
x_{4}=0 .
\end{gathered}
$$

Alle gehen durch die Ecke $x_{1}=x_{2}=x_{3}=0$, aber die drei ersten gehören den drei reellen Kanten dieser Ecke an, während die letate der isolirten Kante zugeordnet ist.

Liegt nun ein Knoten in einer der drei Schnittlinien $x_{1}+x_{2}=$ $x_{1}+x_{3}=0$ etc., so hat die Fläche drei reelle Knoten, kann also nur eine II sein, da die Arten I und I' reelle Pentaeder voraussetzen. 
Flächen, deren 2 reelle Knoten in $x_{4}=0$ liegen, bönnen aber nie weitere erhalten, und darin liegt ein wesentlicher Unterschied, den wir noch genauer erörtern werden.

Uns interessirt zunächst die gewonnene II, da der Process des Verbindens, angewendet auf ihre sämmtlichen Knoten, uns zur gewünschten Ausgangsfläche verhilft.

Zur nothwendigen Bildung der Variationen setzen wir diesmal in der Discriminante:

$$
\alpha_{1}+\alpha_{2}+\alpha_{3}+2 \alpha_{4}
$$

$\alpha_{4}$ negativ, womit allerdings ein Durchsetzen von $x_{4}=0$ ausgeschlossen ist. Dieses ist aber auch nie nothwendig, da beide Seiten genannter Ebene gleichberechtigt sind.

Die Kriterien des Verbindens und Trennens ergeben sich so. Die Fläche mit 3 Knoten hat eben so viele Nullen. Je nachdem man den einen oder den andern Process anwendet, werden die entsprechenden Variationen entgegengesetzte Aenderungen eingeheu. $\mathrm{Da}$ nun ein Trennen - bei allen Knoten - auf eine $V$ führt, welche direct in eine IV' mit isolirtem Kuoten übergeleitet werden kann, so muss jede der Variationen einer solchen Fläche, welche jetzt die Nullen zeigen, gegen die der gesuchten mit 15 Geraden einen Zeichenwechsel aufweisen. Dadurch sind wir aber in den Stand gesetzt, die Kriterien anzugeben, sobald wir nur die Lage der isolirten Knoten kennen. Diese erfiillen aber die Kammer, welche nicht von der isolirten Kante durchsetzt wird, wie man leicht unter Anwendung eines ähnlichen Verfahrens, wie es in $\$ 2$. benutzt wurde, findet, da sich für $\alpha_{1} \alpha_{2} \alpha_{3}$ Grössen mit gleichem Vorzeichen ergeben und. Punkte mit derartigen Coordinaten nach $\$ 1$. der bezeichneten Kammer angehören.

Als für uns brauchbare Speciallä̈chen können wir also hinstellen:

$\left(1,1,1, \frac{1}{2}+i \alpha_{5}, \frac{1}{2}-i \alpha_{5}\right)$ mit 3 Knoten,

$\left(1,1,1, \frac{3}{3}+i a_{5}, \frac{3}{2}-i \alpha_{3}\right)$ mit isolirtem Knoten.

Statt der drei Nullen:

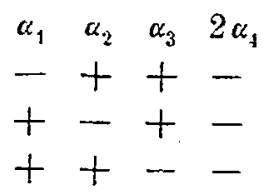

der ersten Fläche, zeigen bei der zweiten dieselben Variationeu negative Zeichen. (Die Null +++- der letztern liefert den isolirten Knoten, der uns augenblicklich nicht weiter beschäftigt.) Folglich haben die angegebenen Variationen bei den Flächen mit 15 Geraden positives Zeicluen. Diese Arten mögen repräsentirt sein durch:

$$
\left(1,1,1, \varepsilon+i \alpha_{i}, \varepsilon-i \alpha_{j}\right) \cdots \varepsilon<\frac{1}{2} \text {. }
$$


Lassen wir das negative Zeichen von $\alpha_{4}$ fort, so giebt das folgende Schema ihre Variationen.
A
B
$\mathrm{C}$
$\alpha_{1} \alpha_{2} \alpha_{3}$
5) $--+\cdots-$
6) $-+-\cdots-$
7) $+--\cdots-$
$\begin{array}{lll}\alpha_{1} & \alpha_{2} & \alpha_{33}\end{array}$
1) $+++\cdots+$

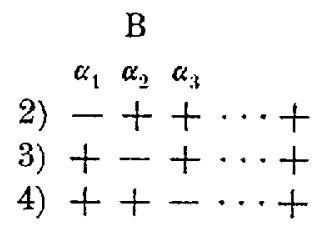

Aus der Bemerkung, dass unter $C$ uor 5) einen Zeicheuwechsel aufweisen kann, der immer durch einen zweiten, 4), unter B begleitet ist, folgt mit Rücksicht auf die bereits discutirten klächen, dass überhaupt nur vier Zeichenwechsel auftreten können.

Die Flächen, welche direct aus unserer II hervorgehen, zeigen offenbar nur Nullen unter B. Wir können uns dann die Null unter A, welche der Fläche mit isolirtem Knoten entspricht, erzeugen, darauf - etwa durch Vergrössern ron $\alpha_{4}$ - diese in einen Zeichenwechsel überführen und haben von nun an keinen Knoten mehr zu erwarten.

Unter unsern Arten findet sich die Diagonalfläche mit drei Zeichenwechseln unter $\mathrm{B}$; sie ist demnach eine $V$.

Die übrigen Flächen müssen sümmtlich inverse sein, sie zeigen den Zeichenwechsel resp. die Null unter C.

Insbesondere: Die Fläche, deren Knoten in der Diagonalebene der isolirten Pentaederkante liegen, ist stets eine II'.

Denn aus ihrer Form:

$$
\left(\alpha_{1}, \alpha_{2}, \alpha_{3}, 0+i \alpha_{5}, 0-i \alpha_{5}\right) \cdots \alpha_{1}+\alpha_{2}+\alpha_{3}=0
$$

erkennt man das Auftreten der Nullen 4) und 5). Durch Veberführung der erstern in einen Zeichenwechsel ergiebt sich die III' mit Knoten, der durch den weitern 5) die IV" folgt, womit die Reihe auch nach dieser Richtung geschlossen ist.

Es bleiben noch die Flächen mit imaginären Knoten zu betrachten übrig.

Eine Anzahl derselben ist unter den behandelten bereits vorhanden, nämlich alle, welche solche Knoten in $x_{4}=0$ haben; bei innen sind die Coefficienten der conjugirten Ebenen reell und positiv.

Um nicht zu weitläufig zu werden, betrachten wir nur eine dieser Artèn, da bei allen die Untersuchung im Wesentlichen dieselbe ist. Liege vor

*) Dass wir hier 7 Variationen und nicht 4 , der Anzahl der Kammern entsprechend, haben, liegt wesentlich darin begründet, dass zwei Knoten in $x_{4}=0$ derselben Kammer angehören, denn es zeigen 4) und 5) gleichzeitig Nullen; die Coordinaten $\alpha_{1} \alpha_{2} \alpha_{3}$ haben sämmtlich entgegengesetzte Zeichen für diese Variationen, was keinen Unterschied hinsichtlich der Kammer bewirkt. 


$$
\left(\alpha_{1}, \alpha_{2}, \alpha_{1}+\alpha_{2}, \alpha_{1}+\alpha_{2}, \alpha_{1}+\alpha_{2}\right)
$$

mit einem isolirten Knoten. Die Schreibweise

$$
\left(\alpha_{1}, \alpha_{2}, \alpha_{1}+\alpha_{2}, 0+i\left(i\left(\alpha_{1}+\alpha_{2}\right)\right), 0-i\left(i\left(\alpha_{1}+\alpha_{2}\right)\right)\right)
$$

lässt aber ausser diesem noch zwei imaginäre Knoten in $x_{4}=0$ erkennen; die Grösse $\alpha_{5}$ ist imaginär.

Man sieht hiernach leicht die Richtigkeit des Satzes ein: Haben die imaginären Ebenen reelle positive Coefficienten und lassen sich die Forzeichen der $\alpha_{1} \alpha_{2} \alpha_{3}$ so wählen, dass ihre Summe $\alpha_{1}+\alpha_{2}+\alpha_{3}$ verschwindet, so besitat die Fläche zwei imaginäre Knoten in der Diagonalebene der isolirten Kunte, zu denen noch so viele reelle Knoten kommen, als Coefficienten reeller Ebenen denen der imaginären gleich sind.

Offenbar brauchen wir uns bei f'eststellung der Art einer gegebenen Fläche hiernach um imaginäre Knoten nicht zu kümmern, sondern haben, sofern ihr Auftreten nicht durch complexe oder negative Coefficienten conjugirter Ebenen ïberhaupt ausgeschlossen ist, nachträglich die angegebene kleine Abzählung vorzunehmen. Ist dann die verlangte Bedingung erfüllt, so haben wir die Uebergangsfäche zwischen zwei allgemeinern derselben Art. -

Alle Flächen mit theilweise negativen $\alpha_{1} \alpha_{2} \alpha_{3}$ sind entweder IV" oder Schläfli'sche IV, 5 mit zwei imaginären Knoten, wie man durch Passiren derjenigen mit einem unendlichen Werthe einer dieser Grössen, wie im § 4., leicht findet. Bemerkt werden muss noch, dass bei festem Pentaeder auch die hierbei zu Tage tretenden Flächen mit imaginären Knoten in Unterabtheilungen gespalten werden müssen, da wir sie hier von IV", im \$ 4. aber ron IV' umgeben finden, und auch unter den IV dieselben sich rorfinden.

In der folgenden Tabelle finden sich neben den Flächen mit reellen resp. ohne Singularitätén diejenigen mit imaginären in Parenthese, welche naan durch continuirliche Aenderung der Coefficienten erzeugen kann, ohne den Zusammenhang zu ändern.

Fläche:

$$
\left(\alpha_{1}, \alpha_{2}, \alpha_{3}, \alpha_{1}+i \alpha_{5}, \alpha_{4}-i \alpha_{5}\right) \ldots \alpha_{1}{ }^{2} \leq \alpha_{2}{ }^{2} \leq \alpha_{3}{ }^{2}, 2 \alpha_{4}<0 .
$$

Variationen einer Fläche mit 15 reellen Geraden:

A

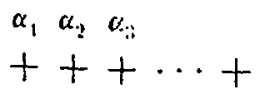

B $a_{1} u_{2} \alpha_{3}$

$-++\cdots+$

$+-+\cdots+$

$++\cdots+$
C

$\alpha_{1} \quad \alpha_{2} \quad \alpha_{: 3}$

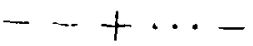


a) Flächen mit positiven Coefficienten reeller Ebenen.

1) Flächen ohne reelle Knoten.

Anzahl der Zeichenwechsel:

0

1 unter B

$2 \eta \mathrm{B}$

1." B, 1 unter C

$3 "$ B

3 " B, 1 unter A
Art:

II

III (oder Schläfli'sehe IV, 6)

IV (oder Sehläfli'sche IV, 5)

IV"

V (oder Schläfli'sche IV, 4)

$1 V^{\prime}$ (oder Schläfli'sche IV, 5)

2) Flächen mit einem reellen Knoten.

Anzahl der Zeichen-

\begin{tabular}{|c|c|c|c|}
\hline $0^{\text {wechsel: }}$ & & $\begin{array}{l}\text { ullen: } \\
\text { ater B }\end{array}$ & $\begin{array}{l}\text { Art: } \\
\text { II }\end{array}$ \\
\hline 1 unter B & 1 & $\eta \quad \mathrm{B}$ & III (oder Schläfli'sche VIII, 5) \\
\hline $1 \# \mathrm{~B}$ & 1 & $\mathrm{C}$ & $\mathrm{III}^{\prime}$ \\
\hline$\eta \mathrm{B}$ & 1 & $\mathrm{~B}$ & IV (oder Schläfli'sche VIII, 4) \\
\hline$\eta \mathrm{B}$ & 1 & A & $I V^{\prime}$ (oder Schläfli'sche VIII, 3) \\
\hline
\end{tabular}

3) Fläcben mit 2 reellen Knoten.

Anzabl der Zeichen-

\begin{tabular}{|c|c|c|c|}
\hline wechsel: & & $\begin{array}{l}\text { allen: } \\
\text { nter B }\end{array}$ & $\begin{array}{l}\text { Art: } \\
\text { II }\end{array}$ \\
\hline & 1 & B, 1 unter C & $\mathrm{II}^{\prime}$ \\
\hline unter B & 2 & $\mathrm{~B}$ & III (oder Schläfli'sehe XVI, 2) \\
\hline
\end{tabular}

4) Flächen mit 3 reellen Knoten.

Anzahl derZeichen-

$\begin{array}{lcc}\text { wechsel: } & \text { Nullen: } & \text { Art: } \\ 0 & 3 \text { unter } \mathrm{B} & \text { II }\end{array}$

b) Flächen mit negativen Coefficienten reeller Ebenen.

1 Coefficient negativ

IV"

2 Coefficienten negativ

IV" (oder Schläfli'sche IV, 5).

\section{$\S 6$.}

Die Flächen mit zwei Paaren conjugirter Pentaederebenen.

Auch in diesem Falle giebt es noch Flächen mit 15 reellen Linien, von denen wir eine zur Ausgangsfläche wählen. Besonders eignet sich hierzu:

$$
\left(x_{2}+i x_{3}\right)^{3}+\left(x_{2}-i x_{3}\right)^{3}+\left(x_{4}+i x_{i}\right)^{3}+\left(x_{4}-i x_{5}\right)^{3}=0 \text {, }
$$

welche in der That der bezeichneten Art angehört. Denu die Schreibweise 


$$
x_{2}\left(x_{2}+x_{3} \sqrt{3}\right)\left(x_{2}-x_{3} \sqrt{3}\right)+x_{4}\left(x_{4}+x_{5} \sqrt{3}\right)\left(x_{4}-x_{5} \sqrt{3}\right)=0
$$

lässt das Vorhandensein der 9 reellen Geraden

$$
x_{2}=x_{4}=0, \quad x_{2}=x_{4}+x_{5} / \overline{3}=0 \text { etc. }
$$

erkennen, folglich sind auch 15 reell, weil andere tlächen obne Singularitäten mit mehr als 9 und weniger als 27 Linien nicht vorkommen. Zur Untersuchung der allgemeinen Fläche

$$
\frac{x_{1}^{3}}{\alpha_{1}^{2}}+\frac{\left(x_{2}+i x_{3}\right)^{3}}{\left(\alpha_{2}+i \alpha_{3}\right)^{2}}+\frac{\left(x_{2}-i x_{3}\right)^{3}}{\left(\alpha_{2}-i \alpha_{3}\right)^{2}}+\frac{\left(x_{4}+i x_{5}\right)^{3}}{\left(\alpha_{4}+i \alpha_{5}\right)^{2}}+\frac{\left(x_{4}-i x_{5}\right)^{3}}{\left(\alpha_{4}-i \alpha_{5}\right)^{2}}=0
$$

oder

$$
\left(\alpha_{1}, \alpha_{2}+i \alpha_{3}, \alpha_{2}-i \alpha_{3}, \alpha_{4}+i \alpha_{5}, \alpha_{4}-i \alpha_{5}\right) \cdots \alpha_{2}^{2} \leqq \alpha_{4}^{2}
$$

mit der Discriminante

$$
\alpha_{1}+2 \alpha_{2}+2 \alpha_{1}
$$

geben wir diesmal $a_{1}$ das feste negative Zeichen, da diese Grösse schon ausgezeichnet ist. Dann finden wir als Vorzeichentafel unserer Ausgangsfiäche $(\infty, 1,1,1,1)$

$$
\begin{aligned}
& 2 \alpha_{2}+2 \alpha_{1} \\
& \text { 1) }++\cdots- \\
& \text { 2) }-+\ldots-
\end{aligned}
$$

Das Zeichen von 3) muss immer mit dem angegebenen überein. stimmen; folglich sind höchstens zwei Zeichenwechsel, entsprechend derselben Anzahl won Trennungen, möglich. Ein Lerfallen in zwei Theile ist also ausgeschlossen: Flächen V, und die ihnen benachbarten IV und IV' mit einem Knoten haben mindestens drei reelle Pentaederebenen.

Nur zwei Diagonalebenen

$$
x_{2}=0, \quad x_{1}=0,
$$

jede eine der beiden isolirten Pentaederkanten enthaltend, sind reell. Sie schneiden sich jedoch in der reellen Pentzederebene und lassen daher nur Flächen mit höchstens 2 reellen Knoten zu.

Es kann aber entweder nur solche Flächen, wie sie aus einer mit 4 Knoten direct bervorgehen, oder inverse geben, da beide Diagonalebenen gleichberechtigt sind, und wir werden die letzte Art erwarten, da schon in vorigen $\$$ die Diagonalebene der isolirten Kante nur Knoten solcher enthält. Diese Vermuthung wird sich als richtig erweisen; damit aber die Untersuchung nicht unterbrochen werde, möge der Beweis später folgen*).

Das Weitere ist dem frühern Verfahren in Wesentlichen gleich und es kann auf die Tabelle verwiesen werden, welche wir am Schlusse geben. Nur die Kriterien imaginärer Knoten erleiden eine geringe

•) Vergl. $\$ 10 .$, p. 79. 
Modification. Die Fälle unter b) der frühern Tabellen kommen in Wegfall, da der Coefficient der einzigen reellen Ebene stets positiv gedacht werden kann. Vorhanden sind imaginäre Knoten demnach nur bei positiven reellen Coefficienten conjugirter Ebenen, und zwar sobald eine oder zwei der Gleichungen

$$
\alpha_{1}-2 \alpha_{2}=0, \quad \alpha_{1}-2 \alpha_{4}=0
$$

erfüllt sind, wie man durch Anwendung der dann noch möglichen Schreibweise der Discriminante (vergl. $\$$ 5.) leicht findet.

Die folgende Tabelle gewährt eine Uebersicht der verschiedenen Arten.

Fläche:

$\left(\alpha_{1}, \alpha_{2}+i \alpha_{3}, \alpha_{2}-i \alpha_{3}, \alpha_{4}+i \alpha_{5}, \alpha_{4}-i \alpha_{5}\right) \cdots \alpha_{2}{ }^{2}<\alpha_{4}{ }^{2}, \alpha_{1}<0$.

Variationen einer Fläche mit 15 reellen Geraden:

$$
\begin{gathered}
2 \alpha_{2} 2 \alpha_{4} \\
\text { 1) }++\cdots-
\end{gathered}
$$

1) Flächen ohne Knoten.

Anzahl der Zeichenwechsel: $\quad$ Art:

0

1

2

II

III (oder Schläfli'sche IV, 6, oder XVI, 3) IV" (ader Schläfli'sche IV, 5).

2) Flächen mit einem reellen Knoten.

Anzahl der Zeichenwechsel: Nullen: Art:

0

1
1

1
II

III' (oder Schläfli'sche VIII, 5).

3) Flächen mit 2 Knoten.

Anzahl der Zeichenwechsel: Nullen: Art:

0

2

II'

\section{$\S 7$.}

Die Scheidung der verschiedenen Mannigfaltigkeiten der Flächen eines Pentaeders.

Durch die bisherigen Untersuchungen ist es möglich geworden, bei gegebenem Pentaeder den Raum so in einzelne Theile zu zerlegen, dass jeder Theil nur die Knoten einer und derselben Flächenart enthält. Die Grenzflächen sind die Diagonal- und Pentaederebenen. Es genügt, die Zerlegung an einer Pentaederkammer mit anschliessender Tetraederkammer zu studiren, da gleichnamige dieser Räume nicht wesentlich von einander verschieden sind. 
Bezeichnen wir die, vorläufig reellen, Ebenen des Pentaeders mit $1,2,3,4,5$, die Diagonalebenen, ibren Gleichungen entsprechend mit zweien dieser Zahlen, so ist auf Tafel I, Fig. 1 die Pentaederkammer, welche vom Trieder 1,2,3 durch die Ebenen 4,5 abgetrennt wird, nebst den sie theilenden Diagonalebenen

$\begin{array}{rr}.14 & 15 \\ 24 & 25 \\ 34 & 35\end{array}$

und zum Theil die Tetraederkammer 1234 zur Anschauung gebracht.

$e$ und $a$ sind Schnittpunkte dreier Diagonalebenen über Tetraederdreiecken, daher Bilder von Flächen mit vier Knoten (nach unserer früheren Interpretation). Die angrenzenden Abtheilungen sind daher Bilder solcher Flächen, wie sie direct aus jener speciellen durch "Trennen" hervorgehen. Ausser diesen Arten kennen wir noch zwei: IV" und III'.

Wirklich giebt es ausser dem bekanntlich IV' entsprechenden Tetraeder 1234 nur noch die gleichartigen Rüune abfh, bcgli, cail, welche nicht von $e$ und $a$ za erreichen sind und daher der Art III' za. kommen müssen.

$d$ gehört dem aufgezeichneten Tetraeder an; wir beschränken uns bei der Untersuchung der einzelnen übrigen Räume auf diejenigen um ¿liesen Punkt, da die Resultate sich später direct auf e übertragen lassen.

Nehmen wir einen isolirten Knoten in 1234 mit den Coordinaten

$$
+\alpha_{1},+\alpha_{2},+\alpha_{3}+\alpha_{4},-\left(\alpha_{1}+\alpha_{2}+\alpha_{3}+\alpha_{1}\right)
$$

und durchsetzen mit ihm die Ebene 4 , aber keine Diagonalebene. Dann ist $\alpha_{4}$ negativ geworden, aber die Grössen $\alpha_{1}+\alpha_{4}, \alpha_{2}+\alpha_{4}, \alpha_{3}+\alpha_{4}$ sind noch positiv. Als neue Coordinaten können wir daher hinstellen

$$
+\alpha_{1},+\alpha_{1},+\alpha_{3},-\delta,-\left(\alpha_{1}+\alpha_{2}+\alpha_{3}-\delta\right),
$$

wo $\delta$ eine in Bezug auf die a sehr kleine Grösse bedeuten mag. Mit Hülfe der Tabelle des $\S 4$. findet man in dieser Fläche eine IV (mit Knoten). Das Durchsetzen der Pentaederebene hat also auf den Knoten tie Wirkung einer Aenderung durch die biplanare Form ausgeibt. Ein jetzt vorzunehmendes Ueberschreiten einer der sich in $d$ schneidenden Diagonalebenen muss aber von einem "Verbinden" begleitet sein, da nur drei solcher Ebenen vorhanden sind und eine I erst durch dreimalige Anwendung jenes Processes auf die IV hervorgeht. Je nachdem ein Knoten also jenseits einer, zweier oder dreier jener Ebenen (aber keiner andern) liegt, ist die Fläche eine III, II oder I.

Nach dieser kleinen Abzählung erkennt man dann die folgende Zerlegung des Raumes. 
Flächen mit einen Knoten.

\begin{tabular}{llc} 
Art: & \multicolumn{1}{c}{ Kammern: } & Anzahl der Kammern: \\
I & $a b c d e$ & $10 \cdot 1=10$ \\
II & $a d f b, a e h b$, etc. & $10 \cdot 6=60$ \\
III & $b d f g, b e h k$, etc. & $10 \cdot 6=60$ \\
III' & $a b f h, b c g k$, etc. & $10 \cdot 3=30$ \\
IV & fgid, hlile, etc. & $10 \cdot 2=20$ \\
IV' & Tetraederkammern &
\end{tabular}

$$
1234 \text { etc. } \frac{5 \cdot 1=5}{185} \text { getrennte Mannigfaltigkeiten. }
$$

Flächen nit zwei Knoten.

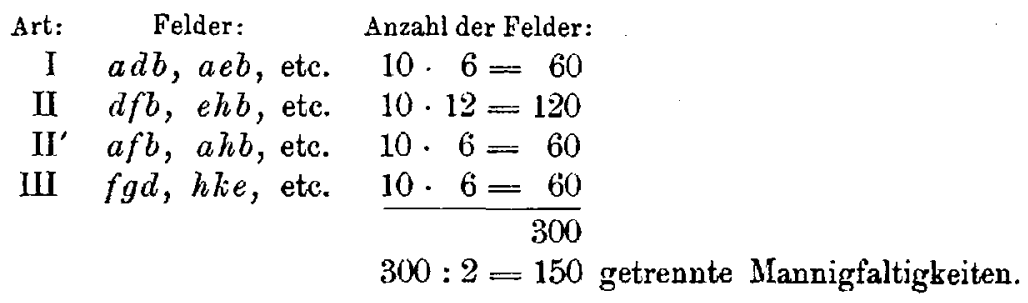

Flächen mit drei Knoten.

Art: Strecken:

I $a d, a e$, etc.

I' $a b, b c$, etc.

II $d f, e h$, etc.
Anzahl der Strecken:

$$
\begin{aligned}
& 10 \cdot 6=60 \\
& 10 \cdot 3=30 \\
& 10 \cdot 6=61\} \\
& \hline 150
\end{aligned}
$$

$150: 3=50$ getrennte Mannigfaltigkeiten.

Flächen mit vier Knoten.

$$
\begin{aligned}
& 10 \cdot 2=20 \\
& 20: 4=5 \text { Flächen. }
\end{aligned}
$$

Wir betrachten noch die Ueberführung der Arten III und III' in einander. Nur diese sind ausser den IV' auf Ränmen abgebildet, welche theilweise won Pentaederebenen begrenzt sind. Aber die begrenzenden Theile dieser Ebenen ( $f b g$ etc. für eine III, $f b h$ etc. für eine III') bilden Scheidewände gegen eine benachbarte Pentaederkammer, ein Uebertritt in diese muss demnach wieder auf eine der Arten III oder III' führen und man findet leicht durch Erweiterung der Figur, dass man, von einer beliebigen der beiden ausgehend, stets die andere erhält. Wir können daher, mit Rüeksicht auf das in Bezug auf die Arten IV und IV' Gefundene, für ein reelles Pentaeder den Satz hinstellen: Liegen die Knoten zweier Flïchen auf verschiedenen Seiten einer Pentaederebene und so, dass man nur diese und nicht etwa 
noch Diagonalebenen zu durchsetzen braucht, um von einem Knoten zum andern zu gelangen, so sind die Flächen stets mit Hülfe der Aenderung durch die biplanare Form in einander iiberfiihrbar.

In ähnlicher Weise lässt sich die Eintheilung des Raumes bei theilweise imaginärem Pentaeder durchführen. Wir beschränken uns auf eine Mittheilung der Resultate.

Tafel I, Fig. 2 giebt eine Centralprojection des Raumes unter Anuahme dreier reellen Pentaederebenen 1, 2, 3, von derem Schnittpunkte auf die Ebene der isolirten Kante. Diese Gerade ist unendlich fern gedacht. Wie unmittelbar zu sehen, ist auch hier noch das Durchsetzèn einer Pentaederebene der Aenderung des Knotens durch die biplanare Form äquivalent.

Ist endlich nur eine Ebene, 1, reell, so schneiden sich diese und die beiden noch reellen Diagonalebenen 23 und 45 in einer Geraden. Tafel I, Fig. 3 zeigt die Projection des von ihnen getheilten Raumes von einem Punkt dieser Geraden auf eine beliebige Ebene, die man passend normal zur Geraden annehmen mag. Ein Ueberschreiten der Pentaederebene ist wirkungslos, denn keine ihrer Seiten ist vor der andern susgezeichnet, was mit dem alleinigen Auftreten inverser Flächen harmonirt.

Der Vollständigkeit wegen fügen wir noch die Aufzählung der einzelnen Abtheilungen bei, wie sie die Figuren ergeben.

Drei reelle Pentaederebenen.

Flächen mit einem Knoten.

Art: Kammern:

II $3 \cdot 1=3$

III $3 \cdot 2 \doteq 6$

III' $\quad 3 \cdot 1=3$

IV $\quad 3 \cdot 1=3$

IV' $\quad 1 \cdot 1=1$

16 getrennte Mannigfaltigkeiten.

Flächen mit 2 wei Knoten.

Art: Felder:

II $3 \cdot 2=6$

II' $\quad 3 \cdot 2=6$

III $3 \cdot 2=6$

18

$18: 2=9$ getrennte Mannigfaltigkeiten.

Flächen mit drei Knoten.

Axt: Gorado:

II $3 \cdot 1=3$

$3: 3=1$ Mannigfaltigkeit. 
Eine reelle Pentaederebene.

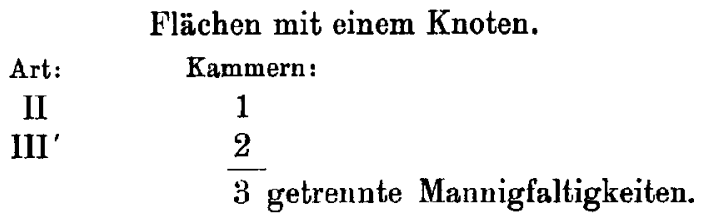

Flächen mit zwei Knoten.

Art: $\quad$ Felder :

II' 4

$4: 2=2$ getrennte Mannigfaltigkeiten.

Was die Eintheilung der Flächen ohue Knoten anlangt, so müssen wir bei derselben absehen von den Hülfsmittelı der Anschauung, wie wir sie bisher benutzt haben, und unsere Resultate auf combinatorischem Wege zu gewinnen suchen, welcher uns übrigens auch bei den erledigten Arten zum Ziele geführt hätte.

Ist eine Fläche gegeben, so kann man sich eine neue, derselben Art, aber einer andern Mannigfaltigkeit angehörig, bei reellem Pentaeder durch Vertauschen beliebiger Ebenen desselben erzeugen. Aber nur das Vertauschen solcher wird von Erfolg sein, deren Coefficienten nicht durch allmähliche Aenderung ihrer Grösse - inzwischen einmal gleich werdend - ihre Plätze wechseln können, ohne einen Wechsel der Art herrorzurûfen.

Die übrigen Coefficienten nimmt man dann aber besser sofort als g]eich an, oder geometrisch: man wählt eine Fläche, auf der sich so oft als möglich drei Gerade in einem Punkte schneiden*). In der Anzahl der dann noch möglichen Permutationen hat man diejenige der getrennten Mannigfaltigkeiten.

Sind nicht alle Ebenen reell, so sind offenbar nur reelle und imaginäre Ebenen unter sich vertauschbar und bei letztern wird weiter nur die Vertauschung zweier Paare conjugirter Ebenen etwas nützen können, da erst hiermit eine Aenderung in der Discriminante eintritt.

Diese Ueberlegungen führen zu der unten mitgetheilten Tabelle. Dieselbe enthält neben der Anzahl der Mannigfaltigkeiten noch die gewählten Repräsentantinnen der verschiedenen Arten. Zur Vermeidung der Angabe von sonst nothwendigen Ungleichungen sei durch $k$ eine sehr kleine Grösse bezeichnet, mit welcher man aber noch all Veränderungen vornehmen kann, welche weder Nullen noch Zeichenwechsel mit sich führen. 


\section{Reelles Pentaeder.}

Art:

$1(\alpha, \alpha, \alpha, \alpha, \alpha)$

II $\left(\alpha, \alpha, \alpha, \frac{3 \alpha+k}{2}, \frac{3 \alpha+k}{2}\right)$

III $(\alpha, \alpha, \beta, \beta, 2 \alpha+k)$

IV $(\alpha, \beta, \beta, \beta, \alpha+\beta+k)$

$I^{\prime \prime}(\alpha, \alpha, 2 \alpha+k, 2 \alpha+l, 2 \alpha+7) \frac{5 !}{2 ! 3 !}=10$

$\mathrm{V}(\alpha, \alpha, \alpha, \alpha, 4 \alpha-k)$

$\operatorname{IV}^{*}(\alpha, \alpha, \alpha, \alpha, 4 \alpha+k)$
Getrennte Mannigfaltigkeiten:

$$
\begin{aligned}
& \frac{5 !}{4 !}=5 \\
& \frac{5 !}{4 !}=5 \\
& \frac{81}{8}
\end{aligned}
$$

Drei Ebenen reell.

$$
\begin{array}{ll}
\text { II }\left(\alpha, \alpha, \alpha, \frac{\alpha-k}{2}, \frac{\alpha-k}{2}\right) & \frac{3 !}{3 !}=1 \\
\text { III }(\alpha, \alpha, 2 \alpha, k+i \beta, k-i \beta) & \frac{3 !}{2 !}=3 \\
\text { IV }\left(\alpha, 2 \alpha, 2 \alpha, \frac{\alpha+k}{2}, \frac{\alpha+k}{2}\right) & \frac{3 !}{2 !}=3 \\
\text { IV' }(\alpha, \alpha, 2 \alpha+k, 0+i \beta, 0-i \beta) & \frac{3 !}{2 !}=3 \\
\text { V }(\alpha, \alpha, \alpha, \alpha, \alpha) & \frac{3 !}{3 !}=1 \\
\text { IV }\left(\alpha, \alpha, \alpha, \frac{3 \alpha+k}{2}, \frac{3 \alpha+k}{2}\right) & \frac{3 !}{3 !}=1
\end{array}
$$

Eine Ebene reell.

$$
\begin{aligned}
& \text { II }(\alpha, k, k, k, k) \\
& \text { III }(\alpha, \alpha, \alpha, \alpha, \alpha) \\
& \text { IV" }(k, k, k, \alpha, \alpha)
\end{aligned}
$$$$
\begin{aligned}
& \frac{2 !}{2 !}=1 \\
& \frac{2 !}{2 !}=1 \\
& 2 !=2 \\
& -\frac{4}{4}
\end{aligned}
$$

$\S 8$.

Ueber ein Verfahren, walches später benutzt werden wird.

Die Uebergangsflächen von denen mit reellem Pentaeder zu denen mit conjugirten Ebenen besitzen eine Doppelebene desselben.

Lässt man zwei Ebenen sich vereinigen, so ist die nächste Folge die Aufbebung der Identität $\Sigma x_{i}=0$, welches Ergebniss-die Unmöglichkeit unsers bisherigen Coordinatensystems mit sich führt. 
Sofern man nicht auf äusserst specielle Flächen kommen will, müssen die Coefficienten der spätern consecutiven Ebenen passend bestimmt, dürfen insbesondere nie endlich gewählt werden, da iu diesem Falle die Fläche, welche sich auf vier Cuben reduciren lässt, hervorgeht, welche, wie sich zeigen wird, bei weitem nicht die allgemeinste Art ist.

Wir betrachten die folgende Aufgabe, welche die von uns zu lösende als speciellen Fall in sich schliesst. Es liege vor die Function

$$
F=\alpha_{1} f\left(x_{1}\right)+\alpha_{2} f\left(x_{2}\right)+\alpha_{3} f\left(x_{3}\right) \cdots+\alpha_{n} f\left(x_{n}\right),
$$

in der die $\alpha$ constante Grössen bedeuten, $f$ eine Function sei, welche sich nach dem Taylor'schen Lehrsatze entwickeln lässt. Wie heisst die allgemeinste Function $F_{n}$, welche entsteht, wenn alle Variabeln $x_{i}$ sich einander unbegrenzt nähern?

Es seien zunächst zwei Functionen $f$ vorhanden:

$$
F=\alpha_{1} f\left(x_{1}\right)+\alpha_{2} f\left(x_{2}\right) \text {. }
$$

Dann machen wir behufs Vereinigung von $x_{1}$ und $x_{2}$ die Transformation

$$
x_{2}=x_{1}+\delta y_{1}
$$

wo $y_{1}$ eine willkürliche Variabele vorstellt, $\delta$ als gegen die Null convergirend anzusehen ist; und entwickeln nach Potenzen von $\delta$ :

$$
\begin{aligned}
F & =\alpha_{1} f\left(x_{1}\right)+\alpha_{2}\left(f\left(x_{1}\right)+\delta f^{\prime \prime}\left(x_{1}\right) y_{1}+\frac{\delta^{2}}{2 !} f^{\prime \prime}\left(x_{1}\right) y_{1}^{2}+\cdots\right) \\
& =\left(\alpha_{1}+\alpha_{2}\right) f\left(x_{1}\right)+\alpha_{2} \delta f^{\prime}\left(x_{1}\right) y_{1}+\frac{\alpha_{2} \delta^{2}}{2 !} f^{\prime \prime}\left(x_{1}\right) y_{1}^{2}+\cdots
\end{aligned}
$$

Nun kann man $\alpha_{1}$ und $\alpha_{2}$ immer derartig wählen - unendlich werden lassen -, dass sowohl $\alpha_{1}+\alpha_{2}$ als auch $\alpha_{2} \delta$ endlich wird. Aber alle weitern Glieder der Entwickelung werden unendlich klein. Man erhält also als gesuchte Function

$$
F_{2}=a_{1} f\left(x_{1}\right)+a_{2} f^{\prime}\left(x_{1}\right) y_{1},
$$

worin $a_{1}$ und $a_{2}$ irgend wie gewählt werden können, dann aber als Constanten zu betrachten sind, während $y_{1}$ eine Variabele ist.

Seien jetzt drei Functionen $f$ gegeben. Dann führt die Transformation

$$
x_{3}=x_{1}+\delta_{1} y_{2}
$$

- unter $\delta_{1}$ eine andere ebenfalls unendlich klein werdende Grösse. verstanden - zu keinem allgemeinern Resultate, sofern man $y_{2}$ willkürlich annimmt. Verläuft aber diese Grösse der Variabeln $y_{1}$ beim Grenzübergange unendlich nahe, so lässt sich eine. Verallgemeinerung erzielen. Wir setzen dem entsprechend:

$$
F=\alpha_{1} f\left(x_{1}\right)+a_{2} f\left(x_{1}+\delta y_{1}\right)+\alpha_{3} f\left(x_{1}+\delta_{1}\left(y_{1}+\delta_{2} y_{2}\right)\right)
$$

und entwickeln wie vorhin: 


$$
\begin{aligned}
F & =\alpha_{1} f\left(x_{1}\right) \\
& +\alpha_{2}\left(f\left(x_{1}\right)+\delta f^{\prime \prime}\left(x_{1}\right) y_{1}+\frac{\delta^{2}}{2 !} f^{\prime \prime}\left(x_{1}\right) y_{1}{ }^{2}+\cdots\right) \\
& +\alpha_{3}\left(f\left(x_{1}\right)+\delta_{1} f^{\prime \prime}\left(x_{1}\right)\left(y_{1}+\delta_{2} y_{2}\right)+\frac{\delta_{1}{ }^{2}}{2 !} f^{\prime \prime}\left(x_{1}\right)\left(y_{1}+\delta_{2} y_{2}\right)^{2}+\cdots\right) \\
& =f\left(x_{1}\right)\left(\alpha_{1}+\alpha_{2}+\alpha_{3}\right)+f^{\prime \prime}\left(x_{1}\right)\left(y_{1}\left(\alpha_{2} \delta^{2}+\alpha_{3} \delta_{1}\right)+\alpha_{3} \delta_{1} \delta_{2} y_{2}\right) \\
& +\frac{f^{\prime \prime}\left(x_{1}\right)}{2 !}\left(y_{1}{ }^{2}\left(\alpha_{2} \delta^{2}+\alpha_{3} \delta_{1}{ }^{2}\right)+2 y_{1} y_{2} \cdot \alpha_{3} \delta_{1}{ }^{2} \cdot \delta_{2}+y_{2}{ }^{2} \cdot \alpha_{3} \delta_{1}{ }^{2} \delta_{2}{ }^{2}\right)+\cdots
\end{aligned}
$$

Nehmen wir jetzt die $\alpha$ unendlich von der zweiten Ordnung, die $\delta$ wie sonst ron der ersten, so werden alle Coefficienten der Producte und Potenzen der $y$ unendlich klein, in denen mehr als zwei Factoren $\delta$ auftreten. Die übrigen bestehen aus endlichen Summanden, oder solchen, welche ron derselben Ordnung unendlich werden. Aber auch die Summen letzterer kann man sich endlich denken, da die Grössen $\alpha$ und $\delta$ durchaus keinen weitern Bedingungen als den hieraus extspringenden unterworfen sind, namentlich nicht als Functionen definirt sind.

Die entspringende Function ist daher

$$
F_{3}=a_{1} f\left(x_{1}\right)+\left(a_{2} y_{1}+a_{3} y_{2}\right) f^{\prime}\left(x_{1}\right)+a_{4} y_{1}^{2} f^{\prime \prime}\left(x_{1}\right) \text {. }
$$

Zur Bildung von $\hat{F}_{\text {, }}$ hätte man analog zu setzen:

$$
\begin{aligned}
F=\alpha_{1} f\left(x_{1}\right)+\alpha_{2} f\left(x_{1}+\delta y_{1}\right) & +\alpha_{3} f\left(x_{1}+\delta_{1}\left(y_{1}+\delta_{2} y_{2}\right)\right) \\
& +\alpha_{4} f\left(x_{1}+\delta_{3}\left(y_{1}+\delta_{4}\left(y_{2}+\delta_{5} y_{3}\right)\right)\right),
\end{aligned}
$$

die $\alpha$ unendlich der dritten Ordnung zu nehmen und in der Entwickelung alle Glieder mit mehr als $3=4-1$ Factoren $\delta$ auszuscheiden - da diese verschwinden - und so fort bei beliebig vielen Functionen.

Heben wir noch hervor, dass die Summe der Zejger aller $y$, welche in einem Product auftreten, stets mit der Anzahl der $\delta$ desselben übereinstimmt, so können wir die Regel hinstellen: beln $x$ aus

Die allgemeinste Function, welche durch Gleichwerden aller Fariaentsteht, ist

$$
F=\alpha_{1} f\left(x_{1}\right)+\alpha_{2} f\left(x_{2}\right)+\cdots+\alpha_{n}\left(x_{n}\right)
$$

$$
\begin{gathered}
F_{*}=f\left(x_{1}\right)+f^{\prime}\left(x_{1}\right) \Sigma y_{i}+f^{\prime \prime}\left(x_{1}\right) \Sigma y_{i} y_{k}+f^{\prime \prime \prime}\left(x_{1}\right) \Sigma y_{i} y_{k} y_{l}+\cdots \\
i \leq k \leq l \cdots,
\end{gathered}
$$

in der die y vollkommen willkürliche Variabeln sind und die Summation so auszuführen ist, dass

ist.

$$
i+k+l+\cdots<n-1
$$

Es sind der Einfachheit wegen die Coefficienten weggelassen, da mau sie in den Variabeln aufgeben lassen kann.

Sollen nicht alle Variabeln einander gleich werden, so hat man nach der angegebenen Regel für die verlangte Anzabl die Function zu bilden und ihr noch die unbetheiligten Functionen $f$ hinzuzufügen. 
$\$ 9$.

Die Flächen mit mehrfachen Pentaederebenen im Allgemeinen.

Die Gleichung einer auf ihr Pentaeder bezogenen Fläche dritter Ordnung hat die Form der Function $F$ des vorigen Paragraphen, der Null gleich gesetzt.

Mittels des angegebenen Satzes sind wir daher im Stande, die Gleichungen von Flächen, deren Pentaeder unendlich nahe Ebenen besitzt, hinzuschreiben, wenn wir $f(x)$ ersetzen durch $x^{3}$.

Abgesehen von der Realität der Elemente sind dann die folgenden Fälle zu unterscheiden:

Pentaeder:

Gleichung der Fläche:

$x_{1}, x_{2}, x_{3}, 2 x_{1}$

$x_{1}, 2 x_{2}, 2 x_{3}$

$x_{1}, \quad x_{2}, \quad 3 x_{3}$

$3 x_{1}, 2 x_{2}$

$4 x_{1}, x_{2}$

$\tilde{\jmath} x_{1}$

$$
\begin{aligned}
& a x_{1}{ }^{3}+b x_{2}{ }^{3}+c x_{3}{ }^{3}+d x_{4}{ }^{3}+c x_{1}{ }^{2} y_{1}=0 \text {, } \\
& a x_{1}^{3}+b x_{2}^{3}+c x_{2}{ }^{2} y_{1}+d x_{3}{ }^{3}+e x_{3}{ }^{3} z_{1}=0 \text {, } \\
& a x_{1}^{3}+b x_{2}^{3}+c x_{3}^{3}+d \dot{x}_{3}{ }^{2}\left(y_{1}+y_{2}\right)+c x_{3} y_{1}^{2}=0 \text {, } \\
& a x_{1}{ }^{3}+b x_{1}{ }^{2}\left(y_{1}+y_{2}\right)+c x_{1} y_{1}{ }^{2}+d x_{2}{ }^{3}+c x_{2}{ }^{3} z_{1}=0 \text {, } \\
& a x_{1}^{3}+b x_{1}{ }^{2}\left(y_{1}+y_{2}+y_{3}\right)+c x_{1}\left(y_{1}{ }^{2}+y_{1} y_{2}\right)+d y_{1}{ }^{3} \\
& +e x_{2}{ }^{3}=0 \\
& a x_{1}{ }^{3}+b x_{1}{ }^{2}\left(y_{1}+y_{9}+y_{3}+y_{4}\right)+c x_{1}\left(y_{1}{ }^{2}+y_{1} y_{2}\right. \\
& \left.+y_{1} y_{3}+y_{2}^{2}\right)+d y_{1}^{3}+e y_{1}^{2} y_{2}=0 \text {. }
\end{aligned}
$$

In diesen Gleichungen bedeuten die $y$ und $z$, der Null gleich gesetxt, Ebenen, die ganz willkürlich angenommen werden können. Sie sind aber nicht, wie die des Pentaeders, eindentig bestimmt, sobald die Gleichung einer Fläche vorliegt. So z. B. wird $y_{1}=0$, ihrer Einführung durch die Gleichung $x_{2}=x_{1}+\delta y_{1}$ (vergl. den vorigen $\$$ ) zufolge, immer durch eine Kante des Pentaeders gehen, nämlich durch die Schnittlinie der consecutiven Ebenen, aber im Uebrigen wird man sie beliebig verändern können. Z. B. kann man in der ersten Gleichung die Substitution

$$
y_{1}=x_{1}+\lambda y_{1}
$$

stets durch Aenderung von $d$ und $e$ wirkungslos machen. Aehnliches gilt auch für die andern hinzukommenden Ebenen.

Gewisse Lagen derselbev wird es jedoch geben, für welcbe die Gleichungen eine besonders einfache Gestalt annehmen. Deren Aufsuchung macht aber die Betrachtung der einzelnen Arten nothwendig, zu der wir uns jetzt wenden.

Die Flächen mit einer Doppelebene des Pentaeders.

Durch die vier getrennt erscheinenden Ebenen ist ein Coordinatentetraeder gegeben. Setzen wir also in der abgeleiteten Gleichung:

$$
\begin{gathered}
a x_{1}{ }^{3}+b x_{2}{ }^{3}+c x_{3}{ }^{3}+d x_{1}^{3}+e x_{4}{ }^{2} y_{1}=0, \\
y_{1}=a_{1} x_{1}+a_{2} x_{2}+a_{3} x_{3}+a_{4} x_{4}
\end{gathered}
$$


so geht diese über in:

$$
a x_{1}{ }^{3}+b x_{2}{ }^{3}+c x_{3}{ }^{3}+\left(d+e a_{4}\right) x_{4}{ }^{3}+e x_{4}{ }^{2}\left(a_{1} x_{1}+a_{2} x_{2}+a_{3} x_{3}\right)=0,
$$

welche sich durch geeignete Wahl der in den $x$ implicite auftretenden Constanten auf die Form

$$
\frac{x_{1}{ }^{3}}{\alpha_{1}{ }^{3}}+\frac{x_{2}{ }^{3}}{\alpha_{2}{ }^{2}}+\frac{x_{3}{ }^{3}}{\alpha_{3}{ }^{2}}-\delta x_{4}{ }^{3}-3 \frac{x_{4}{ }^{2}}{\alpha_{4}{ }^{2}}\left(x_{1}+x_{2}+x_{3}\right)=0
$$

briugen lässt, in $\operatorname{der} x_{1}, x_{2}, x_{3}$ die einfachen Ebenen sind, $x_{4}$ die Doppelebene ist.

Das Verschwinden der Discriminante

ruft den Knoten

$$
\alpha_{1}+\alpha_{2}+\alpha_{3}+\frac{1}{2} d \alpha_{4}^{3}
$$

$$
a_{1}, \alpha_{2}, \alpha_{3}, \alpha_{4}
$$

hervor, dessen Coordinaten demnach nur für die drei ersten und des letzten Giliedes der Gleichung reell. sein können.

Die Grösse $\delta$ ist für die Coordinaten also einflusslos; man hätte sie auch der Einheit gleichmachen können. Da aber dann die speciellen Flächen mit $\delta=0$ ausgeschlossen wären, so musste von dieser Vereinfachung abgesehen werden.

Stellen wir jetzt die Beziehung der Ebene

$$
x_{1}+x_{2}+x_{3}=0
$$

zum Pentaeder fest. Wie bereits im vorigen $\S$ bemerkt wurde, muss ihre Schnittlinie mit $x_{1}=0$ eine Kante desselben und sie selbst, da sie die Ecke der einfachen Ebenen entbält, eine Diagonalebene sein. Um das besser einzusehen und gleich die Frage zu erledigen, ob noch alle Elemente des Pentaeders vollkommen bestimmt sind, bilden wir nach einer von $\mathrm{Clebsch}$ a. a. 0 . angegebenen Methode das Product der 10 Ecken in Ebenencoordinaten $u_{1} u_{2} u_{3} u_{4}$ :

$$
u_{1}^{2} \cdot u_{2}^{2} \cdot u_{3}^{2} \cdot u_{1}\left(u_{1}-u_{2}\right)\left(u_{2}-u_{3}\right)\left(u_{3}-u_{1}\right)=0
$$

Hiermit ist die aufgeworfene Frage bejahend beantwortet, wir fiuden eine Configuration der Ecken, wie sie die Anschauung erwarten liess: Die Schnittpunhte der einfachen Ebenen mit der Doppelebene zählen als Eclien doppelt. $Z u$ diesen kommen in der nämlichen Ebene soch drei cinfache Ecken auf der Linie $x_{4}=x_{1}+x_{2}+x_{3}=0$, was mit der Eigensehaft von $x_{1}+x_{2}+x_{3}=0$ als Diagonalebene im Einklange steht. Mit der Ecke der einfachen Ebenen haben wir die Gesammtzahl aller:

$$
3 \cdot 2+3 \cdot 1+1=10^{\circ} \text {. }
$$

*) Es ist nicht uninteressant, ausgebend von der gefundenen Gleichung der Flïche, das Pentaeder aufzusuchen, welches ohne Schwierigkeit wit Hülfe einiger von Herm Gordan a. a. O. gegebenen Formeln geschehen kann.

Sei

$$
f=a_{x}{ }^{3}=b_{x}{ }^{3}=\cdots=0
$$

die symbolische Darstellung der Gleichung einer Fläche dritter Ordnung, von derem Pentaeder drei Ecken $\xi, \eta$, $\xi$ auf einer Kante gegeben seien. Dann sind die drei Ebenen durch die conjugirte Ecke nach $\$ 16$, III jener Arbeit:

$$
a_{\xi} a_{\eta} a_{x}=0, \quad a_{r_{1}} a_{\zeta} a_{x}=0, \quad a_{\xi} a_{\xi} a_{x}=0
$$


Auf Tafel 11, Fig. 1 ist ein Pentaeder mit zwei sehr nahen Ebenen zur Anschauung gebracht; die später consecutiv werdenden Punkte sind ron geschlossenen Curren umgeben.

Die Fläche besitat im Allgemeinen keine Singularitäten und auch ihre Geraden zeigen keine Besonderheiten in ihrer Lage*). Die Diagonalebenen - ausser $x_{1}+x_{2}+x_{3}=0-$, welche nicht mit der Doppelebene vereinigt liegen, nämlich

$$
x_{1}+x_{2}=0, x_{2}+x_{3}=0, x_{3}+x_{1}=0,
$$

geben aber die Möglichkeit zum Auftreten dreier reellen Knoten, sofern wir $x_{1}, x_{2}, x_{3}$ als reell voraussetzen, was zunächst geschehen soll.

Da Flächen mit 27 reellen Geraden und ihre Grenzflächen mit reellen Knoten stets ein allgemeines Pentaeder voraussetzen, so kann unsere Fläche wit drei Knoten nur eine II sein.

Die Form der Discriminante gestattet uns, das frühere bei der Ableitung der verschiedenen Arten angewendete Verfahren auch jetzt anzuwenden.

Nehmen wir, was keine Beschränkung der Allgemeinheit ist, $\delta$ positiv, $\alpha_{4}$ reell, so haben wir die Fälle reeller oder theilweise imaginärer $\alpha_{1,2,3} \mathrm{zu}$ unterscheiden.

Seien 1) $\alpha_{1} \alpha_{2} \alpha_{3}$ reell und so geordnet, dass $\alpha_{1}{ }^{2} \leq \alpha_{2}{ }^{2} \leq \alpha_{3}{ }^{2}$.

Analog dem Verfahren des $\S 5$. betrachten wir die Flächen:

Nennt man diese $1_{x}, 2_{x}, 3_{x}$, so ist weiter nach $\S 16$, II mit leicht erklarlicher Bezeichnung das Product der übrigen:

$$
a_{x} b_{x}(a b 12)(a b 13)=0
$$

oder, in nicht symbolischer Form, die geränderte Hesse'sche Determinante:

$$
\left|\begin{array}{llllll}
f_{11} & f_{12} & f_{13} & f_{11} & 1_{1} & 3_{1} \\
f_{21} & f_{22} & f_{23} & f_{24} & 1_{2} & 3_{2} \\
f_{31} & f_{32} & f_{33} & f_{34} & 1_{3} & 3_{3} \\
f_{41} & f_{42} & f_{43} & f_{44} & 1_{4} & 3_{4} \\
1_{1} & 1_{2} & 1_{3} & 1_{4} & 0 & 0 \\
2_{1} & 2_{2} & 2_{3} & z_{4} & 0 & 0
\end{array}\right|=0 .
$$

Nimmt man nun als Ecken $\xi \eta \xi$ auf einer Geraden die Punkte $u_{1}-u_{2}=0$, $u_{2}-u_{3}=0, u_{3}-u_{4}$, so geben die mitgetheilten Formeln zunächst die drei Ebenen

$$
x_{1}=0, x_{2}=0, x_{3}=0
$$

und dann mit deren Hülfe für die beiden übrigen

$$
c_{4}^{2}=0 \text {. }
$$

womit das von uns aufgestelite Pentaeder als der Fläche eindeutig zugeordnet nacbgewiesen ist. In ahnlicher Weise kanı mau für die übrigen Arten das Pentaeder bestimmen.

*) Herr Eckardt findet (a. a. O. § 6.) irrthümlich anf diesen Flächen einen uniplanaren Punkt, welches daher rührt, dass die drei einfachen Ebenen und die Doppelebene als durch einen Punkt gehend angenommen werden, indem die Identitiat $x_{1}+x_{2}+x_{3}+x_{4}=0$ als erfüllt angesehen wird. Daon trifft allerdings die angegebene Specialisirung za. (Vergl. \$§ 19., to. der vorliegenden Arbeit.) 
$x_{1}{ }^{3}+x_{2}{ }^{3}+x_{3}{ }^{3}-2 x_{1}{ }^{3}-3 x_{4}{ }^{2}\left(x_{1}+x_{2}+x_{3}\right)=0$ mit drei Knoten, $x_{1}{ }^{3}+x_{2}{ }^{3}+x_{3}{ }^{3}-6 x_{4}{ }^{3}-3 x_{4}{ }^{2}\left(x_{1}+x_{2}+x_{3}\right)=0$ mit.isolirtem Knoten.

Dass der Knoten $(1,1,1,1)$ letzterer Fläche wirklich isolirt ist, erkennt man leicht durch Bildung seines Tangentenkegels, aber auch, wenn man bemerkt, dass er in der Kammer der Ebenen $x_{1}, x_{2}, x_{3}=0$ liegt, welche von der Kante der consecutiven Ebenen $\left(x_{4}=x_{1}+x_{2}\right.$ $+x_{3}=0$ ) nicht durchsetzt wird und dieser Kammer offenbar diejenige solcher Knoten der angrenzenden Flächen mit drei reellen und zwei conjugirten Pentaederebenen entspricht.

Indem wir nun alle möglichen Variationen des Ausdrucks

$$
\alpha_{1}+\alpha_{2}+\alpha_{3}+\frac{1}{2} \delta \alpha_{4}^{3}
$$

aufstellen, finden wir in

$$
x_{1}{ }^{3}+x_{2}{ }^{3}+x_{3}{ }^{3}-3 x_{4}{ }^{2}\left(x_{1}+x_{2}+x_{3}\right)=0
$$

eine Fläche mit 15 Geraden; die hierauf bezügliche Untersuchung, sowie die Ableitung weiterer Flächen "geschieht in der nämlichen Weise wie in $\S 5$., so dass wir uns auf die tabellarische Zusammenstellung (s. den Schluss dieses \$) beschränken können.

Hervorgehoben mag noch werden, dass die ausgezeichnete Diagonalebene $x_{1}+x_{2}+x_{3}=0$ die Knoten der inversen Flächen mit zwei Knoten enthält, was zu erwarten war, da bewiesenermassen nach der. Ueberführung der Doppelebene in zwei imaginäre Ebenen die erwähnte Art der entsprechenden Diagonalebene angehört. -

Zu den Flüchen mit imaginären $\alpha_{1,2,3}$ kann man gelangen durch Ueberschreitung der Fläche

$$
\frac{x_{1}^{3}}{\alpha_{1}{ }^{3}}+\frac{x_{2}{ }^{3}}{\alpha_{2}{ }^{3}}-\delta x_{4}{ }^{3}-3 \frac{x_{4}{ }^{2}}{\alpha_{4}{ }^{2}}\left(x_{1}+x_{2}+x_{3}\right)=0,
$$

für welche $\alpha_{3}$ unendlich ist und welche daher einer Fläche mit imaginüren $\alpha_{3}$ benachbart erscheint.

Die angeführte Gleichung lässt aber del uniplanaren Punkt $x_{1}=x_{2}$ $=x_{\downarrow}=0$ erkennen, welche diese Fläche für uns unbrauchbar macht*).

Es lässt sich dieselbe aber umgehen durch die Annahme $\alpha_{4}=\infty$, welche eine Fläche IV' ohne Knoten hervorruft (\$ 2.), auf welche aber die Vorzeichen der $\alpha^{2}$ ganz ohne Einfluss sind. Folglich sind auch die Flächen mit einem oder zweien imaginären $\alpha_{1,2,3}$ IV', da ein nachheriges Endlichwerden von $\alpha_{4}$ nie einen reellen Knoten erzeugen kann.

*) Solchen Flächen werden wir noch bei anderr Pentaedern unter Voraus. setzung specieller Coefficienten begegnen; wir werden später zeigen (\$ 19.), wie diese Specialformen aus einer allgemeinen sich ableiten lassen. 
Sind insbesondere zwei der imaginären $\alpha$ einander gleich, so hat man die Schläfli'sche IV, 5 mit zwei imaginären Knoten, sofern die Discriminante durch Wahl der übrigen Vorzeichen zum Verschwinden gebracht werden kann. Gepaart können reelle und imaginäre Knoten demnach nicht auftreten.

Wir gehen über zu den Flächen mit zwei conjugirten Ebexen:

$$
\frac{x_{1}^{3}}{\alpha_{1}{ }^{2}}+\frac{\left(x_{2}+i x_{3}\right)^{3}}{\left(\alpha_{2}+i \alpha_{3}\right)^{2}}+\frac{\left(x_{2}-i x_{3}\right)^{3}}{\left(\alpha_{2}-i x_{3}\right)^{2}}-\delta x_{4}^{3}-3 \frac{x_{1}^{2}}{\alpha_{4}{ }^{2}}\left(x_{1}+2 x_{2}\right)=0
$$

mit der Discriminante

$$
\alpha_{1}+2 \alpha_{2}+\frac{1}{2} \delta \alpha_{4}^{3}=0
$$

Die beiden reellen Diagonalebenen

$$
x_{2}=0, x_{1}+2 x_{2}=0
$$

ermöglichen das Auftreten von Flächen mit zwei reellen Knoten, nicht mit dreien, da ihre Sehnittlinie in $x_{1}=0$ liegt.

Man kann folgendermassen nachweisen, dass solehe Flächen immer II' $\operatorname{sind}$.

Es mögen die Knoten zunächst in der Diagonalebene der isolirten Kante: $x_{2}=0$ liegen. Die Flächen mit zwei conjugirten Ebenen eines allgemeinen Pentaeders, deren Knoten in der entsprechenden Diagonalebene liegen, sind immer von der angegebenen Art (\$ 5.). Daher sind es auch die unserigen, da man zwei der reellen Ebenen beliebig nahe bringen kann, ohne die Fläche zu zerstören. Denken wir uns ferner, bei derselben Fläche, zur Erledigung des zweiten Falles die Doppelebene durch eine unendlich kleine Aenderung der Constanten in zwei conjugirte Ebenen übergeführt (was allerdings nicht onter Beibehaltung unserer Gleichungsform möglich ist). Dadurch tritt kein Knoten auf, denn beim jetzigen Pentaeder mit zwei Paaren conjugirter Ebenen können nach \$ 6 . nicht mehr als zwei reelle Knoten vorhanden sein.

Einem solchen Pentaeder kommt aber nur eine Art mit zwei Knoten $2 \mathrm{u}(\$ 6$.$) , diese muss also auch eine II' sein, welches Resultat wir$ daselbst vorausnahmen.

Aber damit ist dasselbe für den Fall zweier Knoten in $x_{1}+2 x_{2}=0$ bewiesen, da wir den Uebergang zu zwei conjugirten Ebenen ebenso gut bei einer solchen Fläche hätten bewirken können.

Durch den Process des Trennens werden wir demnach auf Flächen IV", durch den des Verbindens auf II geführt.

Erstere Art entspringt wirklich bei der Annahme $\alpha_{i}=\infty$. Nimmt man $\alpha_{1}$ negativ, so lauten ihre Variationen, oder besser, die Variationen einer andern IV" mit endlichem $\alpha_{4}$ 


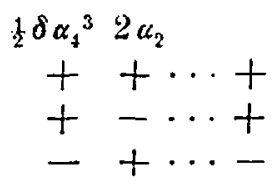

und zwar muss eine der beiden letzten in allen Fällen negativ sein. Durch Wachsenlassen von $\alpha_{1}$ kann man drei negative Zeichen herstellen. Beim Passiren der aufgetretenen Nullen verbanden sich aber nothwendiger Weise Theile, da hierin die einzige Möglichkeit zur Erzeugung der II liegt, welche Art doch vorkommt und somit auch erhalten ist. Das Weitere bedarf keiner Erläuterung.

Für $a_{1}=\infty$ tritt freilich ein uniplanarer Punkt auf, aber diese Grenzfläche brauchen wir nie zu erreichen.

Flächen nit zwei imaginären Knoten kommen unter den eben betrachteten vor, wenn die. Coefficienten der conjugirten Ebenen reell und positiv sind (rergl. $\$ \S 5 ., 6$ ) und

$$
\alpha_{1}+\frac{1}{2} \delta \alpha_{4}^{3}=0
$$

ist. Auch auf diesen können nie reelle Knoten auftreten, wie es vorhin bei drei reellem Pentąederebenen erkannt wurde.

Ist endlich $\alpha_{1}$ imaginür, so bann nie ein Knoten auftreten and die Betrachtung der Fläche mit $\alpha_{4}=\infty$ zeigt, dass jene wie letztere immer eine IV" ist. -

\section{Tabelle I.}

$$
x_{1} x_{2} x_{3} \text { reell. }
$$

Fläche: $\frac{x_{1}{ }^{3}}{\alpha_{1}{ }^{2}}+\frac{x_{2}{ }^{3}}{\alpha_{2}{ }^{2}}+\frac{x_{3}{ }^{3}}{\alpha_{3}{ }^{2}}+\delta x_{1}^{3}-\frac{3 x_{1}{ }^{2}}{\alpha_{1}{ }^{2}}\left(x_{1}+x_{2}+x_{3}\right)=0$,

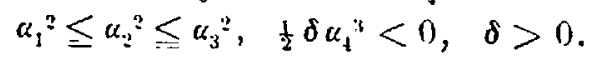

Variatiouen einer Fläche mit 15 reellen Geraden.

A

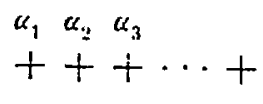

B

$$
u_{1} \alpha_{2} u_{3}
$$$$
-++\cdots+
$$$$
+-+\cdots+
$$$$
++\cdots+
$$

a) Flächen mit positiven Coefficienten reeller Ebenen.

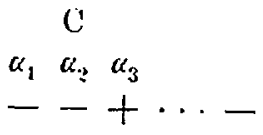

1) Flächen ohne reelle Knoten.

Anzahl der Zeichenwechsel:

0

1 unter B

Art:

$2 》$ B

$1 "$ B, 1 unter C

$3 » \mathrm{~B}$

$3 \%$ B, 1, $\mathbf{A}$ 
2) Flächen mit einem reellen Knoten.

Anzahl der Zeichenwechsel:

Nollen:

0

1 unter $B$

Art:

1 unter B

$1 ”$ B

II

$1, \mathrm{~B}$

$1 » \mathbf{C}$

III

$2 》$ B

$1 ” B$

HI'

3 B

$1 " \mathrm{~A}$

IV

IV'

3) Flächen mit zwei reelten Knoten.

Anzahl der Zeichenwechsel:

Nullen:

0

2 unter B

Art:

0

$1 \%$ B, 1 unter C

II

1 unter B

$2 "$ B

II'

III

4) Flächen mit drei reellen Knoten.

Anzahl der Zeichenwechsel:

0

Nullen:

3 unter B

Art:

II

b) Flächen mit negativen Coefficienten einfacher Ebenen.

1 Coefficient negativ IV'

2 Coefficienten negatir IV' (oder Schläfli'sche IV, 5).

\section{Tabelle II.}

Nur $x_{1}$ reell.

Fläche: $\frac{x_{1}{ }^{3}}{\alpha_{1}^{2}}+\frac{\left(x_{2}+i x_{3}\right)^{3}}{\left(\alpha_{2}+i \alpha_{3}\right)^{2}}+\frac{\left(x_{2}-i x_{3}\right)^{3}}{\left(\alpha_{2}-i \alpha_{3}\right)^{2}}-\delta \alpha_{4}{ }^{3}-3 \frac{x_{4}{ }^{2}}{\alpha_{4}{ }^{2}}\left(x_{1}+2 x_{2}\right)=0$, $a_{1}<0$.

Variationen einer Fläche mit 15 reellen Geraden.

$$
2 u_{2}, \frac{1}{2} \delta u_{4}^{3}
$$

1) Flächen ohne reelle Knoten.

Anzahl der Zeichenwechsel:

0
1
2

Art:

II

III- (oder Schläfli'sche IV, 6)

IV" (oder Schläfli'sche IV, 5).

2) Flächen mit einem reellen Knoten.

Anzahl der Zeichenwechsel: Nullen: Art:

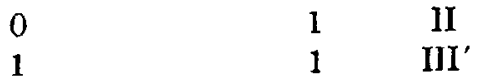

3) Flächen mit zwei Kuoten.

Anzahl der Zeichenwechsel:

0
Nullen:

2

Art:

II' 


\section{$\S 11$.}

Die Flächen mit 2 wei Doppelebenen des Pentaeders.

Nehmen wir $x_{1}=0$ und $x_{2}=0$ zu den Doppelebenen, $x_{3}=0$ zur einfachen, so geht die in $\S 7$. gefundene Gleichung über in

$$
\begin{aligned}
& a x_{1}{ }^{3}+x_{1}{ }^{2}\left(a_{1} x_{1}+a_{2} x_{2}+a_{3} x_{3}+a_{4} x_{4}\right) \\
+ & b x_{2}{ }^{3}+x_{3}{ }^{2}\left(b_{1} x_{1}+b_{2} x_{2}+b_{3} x_{3}+b_{4} x_{4}\right)+c x_{3}{ }^{3}=0 .
\end{aligned}
$$

Da diese die Form

$$
x_{4}\left(a_{4} x_{1}{ }^{2}+b_{4} x_{2}{ }^{2}\right)+f\left(x_{1}, x_{2}, x_{3}\right)=0
$$

hat, so besitzt die Flüche einen biplanaren Knoten im Schnittpunkte der Doppelebenen mit der einfachen und die Ebenen des Knotens

$$
\sqrt{a_{4}} x_{1}+\sqrt{b_{4}} x_{2}=0 \text { und } \sqrt{a_{4}} x_{1}-\sqrt{b_{4}} x_{2}=0
$$

sind harmonisch eu den Doppelebenen*). Aber die Axe des Knotens - der Durchscbnitt seiner Ebenen - liegt nicht auf der Fläche, wir baben die niedrigste Art eines solchen Punktes, einen $B_{3}$, so genannt, weil die Classe einer Fläehe durch sein Auftreten um drei Einheiten erniedrigt wird**). Die vorliegenden Flächen sind Schläfli'sche III und zwar die allgemeinsten.

Es verdient wohl bemerkt zu werden, dass conische Knoten nie von Einfluss auf das Pentaeder sind, während biplanare ganz specielle Pentaeder voraussetzen, aber unter Zugrundelegung dieser völlig umabhängig von der Wahl der Constanten auftreten.

Whe wir zur Unterscheidung der verschiedenen Arten schreiten, sei an die Vertheilung der Geraden auf unsern Flächen erinnert***). Jede Ebene des biplanaren Punktes schneidet die Fläche in drei sich in ihm kreuzenden Geraden (Strahlen), von denen bei reellen Ebenen mindestens in jeder einer reell ist. Sind alle Strahlen reell, so hat die Fläche überhaupt nur reelle Linien. Ein Zusammenrücken zweier Strahlen bewirkt das Auftreten eines conischen Knotens und ein darauf folgendes Imaginärwerden eine Trennung der ihn umgrenzenden Partieen der Fläche. Rücken alle-drei Strahleu zusammen, so entsteht ein weiterer $B_{3}$, deren böchstens drei auftreten können $\dagger$ ). Bei imaginären Ebenen kann durch den Knoten nie ein reeller Strahl gehen und etwaige conische Knoten sind daher stets conjugirt. -

*) Vergl. meine Dissertation $\$ 6$.

**) In gleicher Weise möge stets die Zahl der Einheiten, um welche irgend eine höhere Singularität die Classe erniedrigt, dem bezeichnenden Buchstaben als Zeiger angehängt werden.

*) Vergl. Schläfli a. a. O., Klein a. a. 0. \$ 3., oder Salmon-Fiedler Raumgeometrie II, Art. 267.

†) Solche Flächen verlangen jedoch eine weitere Specialisirung des Pentaeders. Vergl die $\$ \$ 12$, und 17. dieser Arbeit. 
Für das Folgende ist zu unterscheiden, ob die Doppelebenen reell oder conjugirt sind. (Den Uebergang durch eine vierfache Ebene behandeln wir im nächsten \$.)

Bei reellen $x_{1}$ und $x_{2}$ geben wir der Gleichung die Gestalt:

$$
\begin{gathered}
\gamma x_{1}{ }^{3}+\delta x_{2}{ }^{3}+3 \frac{x_{1}{ }^{2}}{\alpha_{1}{ }^{2}}\left(x_{3}+x_{4}\right)+3 \frac{x_{2}{ }^{2}}{\alpha_{2}{ }^{2}}\left(x_{3}-x_{4}\right)-2 \frac{x_{5}{ }^{2}}{\alpha_{3}{ }^{2}}=0, \\
\gamma, \delta>0 .
\end{gathered}
$$

Das Product der Knoten

$$
u_{4}^{4}\left(u_{3}+u_{4}\right)^{2}\left(u_{3}-u_{3}\right)^{2} \cdot u_{1} u_{2}=0
$$

zeigt die Vereinigung von 4 Ecken im biplanaren Punkte, ferner giebt es zwei doppeltzählende auf der Axe und zwei einfache in der einfachen Pentaederebene: $4+2 \cdot 2+2 \cdot 1=10$. Wir haben eine Gruppirung, wie die Anschaunng sie direct ergiebt. (Tafel II, Fig. 2.)

Es ist wichtig, die Ebenen

$$
x_{3}+x_{4}=0 \text { und } x_{3}-x_{1}=0
$$

als, noch übrige, Diagonalebenen der einfachen Ecken zu erkennen, denn sie lassen die Möglichkeit von Flächen mit zwei conischen Knoten einsehen.

Der biplanare Punkt ist selbstrerständlich bei der Aufstellung der Bedingung für einen Knoten auszuschliessen; thun wir dieses, so ergiebt sich als solche

für den conischen Knoten

$$
\gamma \alpha_{1}{ }^{3}+\delta \alpha_{2}{ }^{3}+4 \alpha_{3}=0
$$

$$
\alpha_{1}, \alpha_{2}, \alpha_{3}, \gamma \alpha_{1}{ }^{3}+\delta \alpha_{2}{ }^{3} .
$$

Um bei einer speciellen Fläche leicht die Realitätsverhältnisse der Geraden übersehen zu können, nehmen wir für den Augenblick die Ebenen des Knotens zu Coordinatenebenen und setzen:

$$
\begin{aligned}
& x_{1}^{\prime}=\frac{x_{1}}{\alpha_{1}}+\frac{x_{2}}{\alpha_{2}} \quad x_{1}=\alpha_{1} \frac{x_{1}^{\prime}+x_{2}^{\prime}}{2} \\
& x_{2}^{\prime}=\frac{x_{1}}{\alpha_{1}}-\frac{x_{2}}{\alpha_{2}} \quad x_{2}=\alpha_{2} \frac{x_{1}^{\prime}-x_{2}^{\prime}}{2} .
\end{aligned}
$$

Dann erscheint die Form:

$$
\begin{aligned}
\gamma{\alpha_{1}}^{3}\left(x_{1}{ }^{\prime}+x_{2}\right)^{3}+\delta \alpha_{2}{ }^{3}\left(x_{1}{ }^{\prime}-x_{2}{ }^{\prime}\right)^{3} & +6\left(x_{1}{ }^{2}+x_{2}{ }^{2}\right) x_{3} \\
& +12 x_{1} x_{2} x_{4}-16 \frac{x_{3}{ }^{3}}{\alpha_{3}{ }^{2}}=0 .
\end{aligned}
$$

Unter der Voraussetzang reeller $\alpha$ findet sich in der Fläche mit $\gamma=\delta=0$ :

$$
6 x_{1}^{\prime} x_{2}{ }^{\prime} x_{4}+3\left(x_{1}{ }^{2}+x_{2}{ }^{2}\right) x_{3}-8 \frac{x_{3}{ }^{3}}{\alpha_{3}{ }^{2}}=0,
$$

eine Schläfli'sche III, 1, denn jede der Ebenen $x_{1}^{\prime}=0, x_{q}^{\prime}=0$ schneidet in drei reellen Strahlen.

Die Ableitung der übrigen Arten aus dieser geschieht wie sonst durch Erzeugung von conischen Knoten und darauf folgendes Trennen 
der angrenzenden Partien, resp. Erhaltung beliebig vieler Knoten. Tabelle I, a) enthält die nach diesem Princip gewonnenen Resultate.

Die Flächen mit theilweise imaginären $\alpha$ sind leicht erledigt.

Ist eine der Grössen $\alpha_{1}$ und $\alpha_{2}$ imaginär, so haben wir conjugirte Ebenen des Knotens, Schläfli sche III, 4; ein ausserdem imaginäres $\alpha_{3}$ giebt dieselbe Fläche, auf der aber bei geeigneten Werthen der übrigen Constanten zwei imaginäre Knoten auftreten können. (Tabelle $\mathrm{I}, \mathrm{b})^{*}$ ).

Sind endlich $\alpha_{1}$ und $\alpha_{2}$ imaginär oder, was dasselbe ist, ist bei reellen $\alpha_{1}$ und $\alpha_{2} \alpha_{3}$ imaginär, so erhält man stets in jeder der jetzt reellen Ebenen des Knotens einen reellen Strabl, wie die Specialfläche $\gamma=\delta=0$ zeigt. Denn eine Aenderung kann nicht eintreten der Unmöglichkeit reeller Knoten wegen. (Tabelle I, c.)

Seien jetzt die Doppelebenen conjugirt. Dann sind es auch die Diagonalebenen, und zwei reelle Knoten können nicht auftreten. Da ferner die Coefficienten $\alpha_{1}$ und $\alpha_{2}$ conjugirt sein müssen und so die Möglichkeit eines einzigen reellen wegfällt, so lassen wir beide in $x_{1}$ und $x_{2}$ aufgehen, da sonst die Criterien der verschiedenen Arten minder einfach werden würden, und geben der Gleichung die Form:

$(\gamma+i \delta)\left(x_{1}+i x_{2}\right)^{3}+(\gamma-i \delta)\left(x_{1}-i x_{2}\right)^{3}+3\left(x_{1}+i x_{2}\right)^{2}\left(x_{3}+i x_{4}\right)$

$$
+3\left(x_{1}-i x_{2}\right)^{2} \cdot\left(x_{3}-i x_{4}\right)-2 \frac{x_{3}^{3}}{\alpha_{3}^{2}}=0 \text {. }
$$

Von Ecken bleibt nur reell der vierfach zählende biplanare Punkt, wie das abgeleitete Product für die jetzigen Elemente ergiebt.

Die Ebenen jenes Knotens sind

$$
x_{1}=0, x_{2}=0 \text {, }
$$

folglich stets reell, wodurch auch imaginäre conische Knoten ausgeschlossen sind. Bei conjugirten Doppelebenen des Pentaeders sind die Elenen des biplanaren Knotens reell und es treten nie zwei conische Knoten auf.

Da höcbstens in einer Ebene des $B_{3}$ arei reelle Strahlen liegen künnen, so bewirkt ein Durchgang durch einen Knoten die Ueberfübrung dieser Arten in solche, welche in jeder Ebene nur eimen reellen Strahl aufweisen.

Die Bedingung für das Auftreten eines Knotens hat die Form

$$
\gamma+2 \alpha_{3}=0
$$

angenommen. Seine Coordinaten sind:

$$
x_{1}=1, \quad x_{:}=0, \quad x_{3}=\alpha_{3}, \quad x_{4}=\gamma \text {. }
$$

*) Die Uebergangstächen mit $\alpha_{2}=\infty$ zeigen einen uniplanaren Punkt und diejenigen mit $\alpha_{3}=\infty$ sind Regelflichen. Vergl. für die erstern $\$ 19.2$ för die
letatern $\$ 18$. 
Es kann also nur eine Veränderung in $x_{2}=0$ bein Passireu des Knotens vor sich gehen, und $x_{1}=0$ enthält demnach immer nur einen reellen Strahl.

Da nun bei reellem $\alpha_{3}$ auf der Fläche mit $\gamma=0$ sich in $x_{2}=0$ drei reelle Strahlen vorfinden, so baben alle Flächen, bei deren Äbleitung aus dieser sich ${ }^{\circ}$ ein Knoten nothwendig macht, in derselben Ebene (also in jeder) nur einen reellen Strahl. (Tabelle II, a). Zur letztern Art gehören auch alle mit imaginärem $\alpha_{3}$, wie dasselbe Beispiel zeigt, da der zur Abänderung nothwendige Knoten nicht auftritt. (Tabelle II, b.)

\section{Tabelle I.}

Reelle Doppelebenen.

Fläche: $\gamma x_{1}{ }^{3}+\delta x_{2}{ }^{3}+\frac{3 x_{1}{ }^{2}}{\alpha_{1}{ }^{2}}\left(x_{3}+x_{4}\right)+\frac{3 x_{2}{ }^{2}}{\alpha_{2}{ }^{2}}\left(x_{3}-x_{1}\right)-2 \underset{\alpha_{3}{ }^{2}}{x^{3}}=0$, $\gamma, \delta>0$

a) $\alpha_{1}^{2}, \alpha_{12}{ }^{2}, \alpha_{3}^{2}$ positiv.

$$
\gamma \alpha_{1}^{3}<\delta \alpha_{2}^{3}, \quad \alpha_{3}<0 .
$$

Variationen von $\gamma \alpha_{1}{ }^{3}+\delta \alpha_{2}{ }^{3}+4 \alpha_{3}$ einer Fläche mit lauter reellen Geraden:

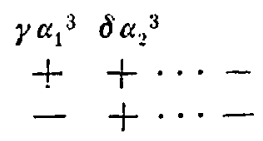

1) Flächen ohne reelle conische Kuoten. Anzahl der Zeicheu-

wechsel:
0

1

2

1

2) Flächen Anzahl der Zeichenwechsel: 1
Art:

In jeder Ebene drei reelle Strahlen (Schläfli'sche III, 1) "einer $"$ keiner $" \quad " \quad "$
III, 2) III, 3) $n$

3) Flächen nit zwei Knoten. Anzahl der Zeichenwechsel: Nullen:

Nullen:

1 1

Art:

In der unbetheiligten Ebene liegen drei reelle Strahlen (Schläfli'sche VI, 1) ein reeller Strahl (Schläfli'sche VI, Z)

Art: 0 2

Alles reell (Schläfli'sche XIII, 1).

b) $\alpha_{1}^{2}$ oder $\alpha_{2}^{2}$ negativ, $\alpha_{3}^{2}$ positiv.

Die Ebenen des $B_{3}$ sind conjugirt. Schläfli'sche III, 4 (oder XIII, 2 mit 2 imaginären Knoten).

c) $\alpha_{3}^{2}$ negativ, oder $\alpha_{1}^{2}$ und $\alpha_{2}^{2}$ negativ.

Schläfli'sche III, 3 . 


\section{Tabelle II.}

Conjugirte Doppelebenen.

Fläche: $(\gamma+i \delta)\left(x_{1}+i x_{2}\right)^{3}+(\gamma-i \delta)\left(x_{1}-i x_{2}\right)^{3}$

$$
\begin{aligned}
& +3\left(x_{1}+i x_{2}\right)^{2}\left(x_{3}+i x_{4}\right) \\
& +3\left(x_{1}-i x_{2}\right)^{2}\left(x_{3}-i x_{4}\right)-2 \frac{x_{3}{ }^{3}}{\alpha_{3}{ }^{2}}=0 .
\end{aligned}
$$

a) $\alpha_{3}{ }^{2}$ positiv.

Die Fläche ist, wenn dem absoluten Werthe nach $\gamma \lesseqgtr 2 \alpha_{3}$, eine

b) $\alpha_{3}^{2}$ negativ.

$$
\text { Schläfli'sche }\left\{\begin{array}{l}
11 \mathrm{I}, 2 \\
\text { VI, } 2 \\
\text { III, } 3 .
\end{array}\right.
$$

Schläfli'sche III, 3 .

\section{$\$ 12$.}

Die Flächen mit vierfacher Pentaederebene.

Sie bilden den Uebergang zwischen den Flächen mit reellen Doppelebenen einerseits und conjugirten andererseits, und werden daher ebenfalls einen biplanaren Punkt aufweisen. Daher erscheint es gerechtfertigt, dieselben vor denen mit dreifacher Ebene zu betrachten, wenn auch letztere allgemeinerer Natur sind.

Behält max in der abgeleiteten Gleichung

$$
a x_{1}{ }^{3}+b x_{1}{ }^{2}\left(y_{1}+y_{2}+y_{3}\right)+c x_{1}\left(y_{1}{ }^{2}+y_{1} y_{2}\right)+d y_{1}{ }^{3}+e x_{2}{ }^{3}=0
$$

die vierfache Ebene $x_{1}=0$ und die einfache $x_{2}=0$ als Coordinatenebenen bei, setzt vorläufig

$y_{1}+y_{2}+y_{3}=x_{4}, \quad y_{1}=x_{3}, \quad y_{1}+x_{2}=a_{1} x_{1}+a_{2} x_{2}+a_{3} x_{3}+a_{4} x_{4}$, so lässt die entspringende Form

$$
a x_{1}{ }^{3}+b x_{1}{ }^{2} x_{4}+c x_{1} x_{3}\left(a_{1} x_{1}+a_{2} x_{2}+a_{3} x_{3}+a_{4} x_{4}\right)+d x_{3}{ }^{3}+e x_{2}{ }^{3}=0
$$

schon den $B_{3}: 0,0,0,1$ erkennen, dessen Ebenen sind:

$$
x_{1}=0, \quad b x_{1}+c a_{4} x_{3}=0 \text {. }
$$

Folglich ist die vierfache Ebene eine Ebene des stets auftretenden $B_{3}$, wie zu erwarten war, da im allyemeinen Falle die Ebenen eines solchen Punktes zu den Doppelebenen des Pentaeders harmonisch sind.

Nimmt man die andere: $b_{1} x_{1}+c a_{4} x_{3}=0$ zu $x_{3}=0$, so kann man durch passende Veränderung von $x_{4}$ die Gleichung zu

vereinfachen.

$$
\beta x_{1}^{3}-\frac{3 x_{1}^{2} x_{2}}{\alpha_{1}^{2}}+6 x_{1} x_{3} x_{4}+x_{3}^{3}+p x_{3}^{3}=0
$$

Flächen, bei denen eins der drei letzten Glieder fehlt, sind entweder Kegel oder haben einen uniplanaren Punkt oder zwei biplanare. 
Nur bei der letztern, für welche $p=0$ ist, sind noch die Ebenen des Pentaeders bestimmt, wenn auch, wie sich zeigen wird, die Ecken in gewisser Weise willkürlich sind.

Die Fläche mit $\alpha_{1}=\infty$ hat eimen osculirenden Kegel, dessen Berührungscurve in $x_{2}=0$ liegt. Für das Folgende ist es wichtig, in ihr eine Schläfli'sche III, 3 zu erkennen, jede Ebene des Knotens enthält einen reellen Strahl.

Für die allgemeine Fläche der vorliegenden Gattung findet man als Product der Pentaedereclien

$$
u_{4}^{6} \cdot u_{2}^{4}=0
$$

ausser dem sechsfach zählender $B_{3}$ findet sich noch ein vierfacher auf $\operatorname{der}$ Axe: $1 \cdot 6+1 \cdot 4=10$.

Die Vermuthung, dass eine allgemeinere Lage der Ecken (z. B. 10 einfache in der vierfachen Ebene, welche Anordnung sich offenbar herstellen lässt) zu allgemeineren Flächen führen müsse, erweist sich als irrig mit Rücksicht auf den Gang der Entwickelung in $\$ 8$. Es ist auch folgendermassen einzusehen, dass jedes andere Eckensystem zu gewissen Unbestimmtheiten führen würde. Bekanntlich ist jeder Ecke eine Kante als Doppelgerade ihrer zerfallenden Polare zugeordnet, was jedenfalls bei völliger Bestimmtheit aller Elemente des Pentaeders voraussetzt, dass die einander entsprechenden auch gleich oft zählen. Bei obiger fingirter Annahme 10 einfacher Ecken erhalten wir aber (wie stets) die vierfache Schnittlinie der beiden getrennt erscheinenden Pentaederebenen (der einfachen und der vierfachen), wodurch ein eindeutiges Entsprechen unmöglich gemacht ist. Aehnliche Unzuträglich. keiten ergeben sich bei allen andern Annahmen mit Ausnahme der gefundenen Configuration. Denn bei dieser ist, wie die Polarenbildung zeigt, dem vierfachen Punkte die erwähnte vierfache Linie und dem sechsfachen die sechsfache Linie der consecutiven Ebenen, die Axe des Knotens, zugeordnet. -

Diagonalebenen giebt es nicht mehr, es kann demnach höchstens ein conischer Knoten auftreten.

Ein solcher ist vorhanden, wenn und hat als Coordinaten

$$
\beta \alpha_{1}^{3}-2=0 \text {, }
$$

$$
\alpha_{1}, 1,0,0 \text {. }
$$

Die Classification geschieht jetzt durch Zugrundelegen der Fläche mit $\boldsymbol{\beta}=0$, welche bei reellem $\alpha_{1}$ von $x_{3}=0$ in drei reellen Strahlen getroffen wird. $\mathrm{Da}$ in $x_{1}=0$ stets nur ein reeller Strahl vorhanden ist, so bewirkt ein Passiren des Knotens den Uebergang von Schläfli'schen III, 2 zu III, 3 (Tabelle I).

Die Flächen mit imaginärem $\alpha_{1}$ sind sämmtlich Schläfli 'sche III, 3, wie die am Anfange dieses $\S$ betrachtete Uebergangsfläche mit $\alpha_{1}=\infty$. 
Wir gehen über zu den Flächen mit zwei biplanaren Knoten $(p=0)$ :

$$
\beta x_{1}^{3}-\frac{3 x_{1}^{2} x_{2}}{\alpha_{1}^{2}}+6 x_{1} x_{3} x_{4}+x_{2}^{3}=0,
$$

deren $B_{3}$ sind: $0,0,0,1$ und $0,0,1,0$.

Dass ein Auftreten zweier solcher Punkte stets eine vierfache Pentaederebene mit sich führt und daher eine jede derartige Fläche durch eine Gleichung unserer Form dargestellt werden kann, liegt darin begründet, dass die Axe eines biplanaren Knotens immer mit der Schnittlinie zweier Doppelebenen zusammenfällt, welche Bedingung eben nur durch ihre weitere Vereinigung zur vierfachen erfüllt sein kann. Flächen mit drei $B_{3}$ können kein bestimmtes Pentaeder mehr haben.

Die Ecken sind aber schon bei den vorliegenden unbestimmt, denn ihr Product, nach der angegebenen Methode gebildet, verschwindet identisch.

In der That sind auch die Gleichungen, welche durch Nullsetzen der Unterdeterminanten der Hesse'schen Determinante:

$$
H=\left\{\begin{array}{rrrr}
\beta x_{1}-\frac{x_{2}}{\alpha_{1}{ }^{2}} & -\frac{x_{9}}{\alpha_{1}{ }^{2}} & x_{4} & x_{3} \\
-\frac{x_{1}}{\alpha_{1}{ }^{2}} & x_{2} & 0 & 0 \\
x_{4} & 0 & 0 & x_{1} \\
x_{3} & 0 & x_{1} & 0
\end{array}\right.
$$

entstehen, für einen beliebigen Punkt der Geraden

$$
x_{1}=x_{2}=0
$$

erfüllt. Alle Punhte der Schnittlinie der beiden getrennt erscheinenden Pentaederebenen haben die Eigenschaft der ehemaligen Pentaederecken. Ausser diesen findet sich noch als völlig bestimmte Ecke der Schnitt der beiden Axen: $0,1,0,0$.

Die Lage der Kanten (wir gebrauchen jetzt noch wie auch in $\mathrm{Zu}$ kunft beim Auftreten unbestimmter Elemente die alten Namen) erschliessen wir durch Polarenbildung.

Als Polaren eines Punktes $0,0, y_{3}, y_{4}$ der Geraden $x_{1}=x_{2}=0$ (Tafel II, Fig. 4) erhält man das Ebenenpaar

$$
x_{1}\left(y_{3} x_{4}+y_{4} x_{3}\right)=0
$$

Deren Doppelgerade, die conjugirte Kante jenes Punktes, enthält die noch bestimmte Ecke. Alle jene Linien bilden daher ein Büschel in der vierfachen Ebene mit letztgenanntem Punkte als Centrum. Auf jeder Kante liegt aússer dem Centrum also noch eine Ecke der Geraden $x_{1}=x_{2}=0$; für die fixirte Kante ist diese $0,0, y_{3},-y_{4}$. Diese Punkte und die Pole bilden folglich eine Involution, deren Doppel- 
punkte die $B_{3}$ sind, so dass allemal die conjugirte Kante eines solchen Punktes ihn selbst enthält, wie es im Falle eines einzigen ist. Die ausgezeichnete Ecke hat die Polare

$$
\left(\frac{x_{1}}{\alpha_{1}}+x_{2}\right)\left(\frac{x_{1}}{\alpha_{1}}-x_{2}\right)=0 \text {, }
$$

d. h. ihre conjugirte Kante enthält die variabeln Ecken.

Nur wenn $\alpha_{1}=\infty$, wird letztere Kante ganz unbestimut; die Fläche hat einen osculirenden Kegel, wie bereits bemerkt wurde*). -

Was die Flächen selbst anlangt, so bleibt die Bedingung für das Auftreten eines conischen Knotens $\beta \alpha_{1}{ }^{3}-2=0$ unverändert, aber bei der Eintheilung haben wir die Fälle reeller oder conjugirter $B_{3}$ zu trennen.

Bei reellen $B_{3}$ ist die Untersuchung durchaus nicht von der frühern verschieden, wir können auf Tabelle II, a) verweisen.

Bei conjugirten $B_{3}$ treten an Stelle von $x_{3}$ und $x_{4}$ resp. $x_{3}+i x_{4}$ und $x_{3}-i x_{4}$; die Gleichung hat die Form:

$$
\beta x_{1}^{3}-\frac{3 x_{1}^{2} x_{2}}{\alpha_{1}^{2}}+3 x_{1}\left(x_{3}^{2}+x_{4}^{2}\right)+x_{2}^{3}=0 .
$$

Der mögliche conische Knoten bann jetzt isolirt sein. Das Auftreten von zwei Arten von Flächen mit Knoten rührt daher, dass zwei im Zeichen von $\beta$ verschiedene Flächen nicht mehr in einander transformirt werden können. Man erkennt auch sofort durch Bildung des Tangentenkegels im Knoten:

$$
\left(\frac{x_{1}}{\alpha_{1}}+x_{2}\right)^{2}+\alpha_{1}\left(x_{3}{ }^{2}+x_{4}{ }^{2}\right)=0,
$$

dass derselbe nur reell, wenn $\alpha_{1}$ negativ und in Folge der Bedingung für sein Auftreten auch $\beta$ negativ ist. Folglich: Bei positivem $\beta$ erhält man Wlächen mit isolirtem (Schläfli'sche XVII, 2), bei negativem $\beta$ Flächen mit nicht isolirtem Knoten (Schläfli'sche XVII, 3).

Hinsichtlich der weitern Eintheilung heben wir hervor, dass die reelle Gerade der conjugirten Ebenen der beiden Knoten : $x_{3}=x_{4}=0$ die Fläche in drei Punkten schneidet, in welchen sich die drei (imaginären) Geradenpaare begegnen, welche in jenen Ebenen liegen. Diese Geradenpaare bestimmen die drei dreifachen Tangentenebenen, welche ausser $x_{1}=0$ noch durch die Verbindungslinie der Knoten $\left(x_{1}=x_{2}=0\right)$ gehen und deren Realitätsverhältnisse die Art der Fläche bedingen ${ }^{* *}$ ). Die Frage nach letzterer ist also anf die einfachere nach der Zahl der reellen jener Punkte zurückgeführt.

Sei zunächst $\alpha_{1}$ reell; dann finden wir bei der Fläche $\beta=0$ nach dem eben Gesagten drei reelle Ebenen. Ein wachsendes $\beta$ kann einen

*) Vergl. die Anmerkung auf der folgenden Seite, вowie \$ 17.

**) Vergl. Schläfli a, a. O. IX. 
isolirten, ein abnehmendes einen nicht isolirten Knoten erzeugen, woraus zu entnehmen, dass die vorliegende, sowie alle zwischen den beiden mit Knoten behafteten Flächen aus zwei Theilen bestehen. Bei weiterer Aenderung verschwindet der Knoten, wenn $\beta$ positiv, im andern Falle werden Theile verbunden.

Während nun derartig erzeugte Flächen ohne jede Singularität und bestimmtem Pentaeder bei Erhaltung desselben (IV' und IV) nicht in einander übergeführt werden konnten, ohne Dazwischentreten vou Knoten, ist dieses bei den unserigen möglich, wie wir gleich sehen werden.

Ein Uebergang durch $\alpha_{1}=\infty$ zu imaginärem $\alpha_{1}$ ändert die Art nicht, alle Flächen sind IX, 4 , denn nie tritt ein reeller Knoten auf. Eben bei diesen Flächen können wir daher $\beta$ beliebig variiren lassen, ohne neue Arten herbeizuführen, womit dann die vorhin behauptete Möglichkeit der Ueberleitung solcher Flächen in einander constatirt ist, die sich im Vorzeichen ron $\beta$ unterseheiden und aus einem Theile bestehen. Damit kommt dann aber auch die Unterscheidung der allgemeinen Flächen IV' und IV in Wegfall, sofern man das Pentaeder unbeachtet lässt, da man imaginäre Singularitäten stets umgehen kann*). -

\section{Tabelle I.}

Alle Elemente des Pentaeders bestimmt. Ein $B_{3}$.

Fläche: $\beta x_{1}{ }^{3}-\frac{3 x_{1}^{2} x_{2}}{\alpha_{1}^{2}}+6 x_{1} x_{3} x_{4}+x_{2}{ }^{3}+p x_{3}{ }^{3}=0$.

1) $\alpha_{1}^{2}$ positiv.

$x_{3}$ enthält $\left\{\begin{array}{ll}3 \text { reelle Strahlen (Schläfli'sche III, } 2), \\ 1 \text { reellen Knoten ( } & \text { VI, 2), } \\ 1 \text { reellen Strahl ( } & \text { III, 3), }\end{array}\right\}$ weun $\beta \alpha_{1}{ }^{3}-2 \leqq 0$.

2) $\alpha_{1}^{2}$ negativ.

Schläfli'sche III, 3.

*) Es mag gestattet sein, hier einige metrische Betrachtungen einzufechten, Nimmat mau bei den Flächen mit zwei conjugirten $B_{3} x_{2}=0$ parallel mit $x_{1}=0$, indem man set2t $x_{k}=x_{1}-h$, nimmt ferner $x_{1}, x_{3}, x_{4}$ als Ebenen eines rechtwinkligen Coordinatensystems, so erkennt man in

$$
\beta x_{1}^{3}-\frac{3 x_{1}^{2}\left(x_{1}-h\right)}{\alpha_{1}^{2}}+3 x_{1}\left(x_{3}^{2}+x_{4}^{2}\right)+\left(x_{1}-h j^{3}=0\right.
$$

die Gleichung einer Fläche, welche durch Ratation einer symmetrischen Curve dritter Ordnung um ihre Symmetrieaxe $x_{3}=x_{4}=0$ entstanden ist. Die $B_{3}$ liegen auf der unendlich fernen Geraden von $x_{1}=0$ und werden bestimmt durch alle Kreise, welche der Parallelbüschel zu dieser Ebene aus der Fläche ausschneidet. Folglich: Jede Rotationsfläche dritter Ordnung hat die imaginären Kreispunkte ihrer Asymptotenezene $z u B_{3}$, Dieser Satz ist umkehrbar. Schneiden sich nun bei der erzeugenden Curve die drei reellen Wendetin-
genten in einem Punkte (der natürlich auf der Rotationsaxe liegt), so geht von 


\section{Tabelle II.}

Ecken und Kanten theilweise unbestimmt. Zwei $B_{3}$.

a) Reelle $B_{3}$.

$$
\text { Fläche: } \beta x_{1}^{3}-\frac{3 x_{1}^{2} x_{2}}{\alpha_{1}^{2}}+6 x_{1} x_{3} x_{1}+x_{2}^{3}=0 \text {. }
$$

1) $\alpha_{1}^{2}$ positiv.

Die Ebenen $x_{3}$ und $x_{4}$ enthalten jede

$$
\left.\begin{array}{lrr}
\text { drei reelle Strahlen (Schläfli'sche IX, 1), } \\
\text { einen Knoten } & (- & \text { XVII, 1), } \\
\text { einen reellen Strahl } & ( & \text { IX, 2), }
\end{array}\right\}
$$

2) $\alpha_{1}^{2}$ negativ.

$$
\text { Schläfli'sche IX, } 2 .
$$

b) Conjugirte $B_{3}$.

Fläche: $\beta x_{1}{ }^{3}-\frac{3 x_{1}{ }^{2} x_{2}}{\alpha_{1}{ }^{2}}+3 x_{1}\left(x_{3}{ }^{2}+x_{1}{ }^{2}\right)+x_{2}{ }^{3}=0$.

1) $\alpha_{1}^{2}$ positiv.

Bedeutet $\beta \alpha_{1}^{3}$ den absoluten Werth dieser Grösse, so erhält man, wenn $\beta a_{1}{ }^{3}-2 \gtreqless 0$ :

eine dreifache Ebene durch $x_{1}=x_{2}=0 \quad$ (Schläfli'sche IX, 4),

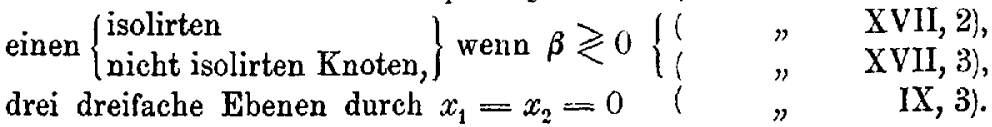

2) $\alpha_{1}^{2}$ negativ.

Schläfli'sche IX, 4.

\section{$\S 13$.}

Die Flächen mit dreifacher Pentaederebene.

Nimmt man zur Vereinfachung ihrer Gleichung

$$
a x_{1}{ }^{3}+b x_{2}{ }^{3}+c x_{3}{ }^{3}+d x_{3}{ }^{2}\left(y_{1}+y_{2}\right)+e x_{3} y_{1}{ }^{2}=0
$$

unter Beibehaltung von $x_{3}=0$ als dreifache und $x_{1}, x_{2}=0$ als einfache Ebenen, als solche des Coordinatensystems

so entsteht die Form:

$$
\begin{aligned}
& y_{1}=x_{1}, \\
& y_{2}=x_{1}+x_{2}-x_{1},
\end{aligned}
$$

$$
\frac{x_{1}^{3}}{\alpha_{1}{ }^{2}}+\frac{x_{2}^{3}}{\alpha_{2}^{2}}+\gamma x_{3}{ }^{3}-3 \frac{x_{3}^{2}\left(x_{1}+x_{2}\right)}{\alpha_{3}^{2}}-3 x_{3} x_{4}^{2}=0 \cdots \alpha_{1} \leq \alpha_{2},
$$

diesem ein osculirender Kegel an die Fläche, dessen Berührungscurve aub einem Kreise, in $x_{1}=h$, and der unendlich fernen Geraden besteht. Wir haben den Fall $\alpha_{1}=\infty$. 
in der wir $\alpha_{3}^{2}$ stets als positiv voraussetzen könuen, ohne die Allgemeinheit $\mathrm{zu}$ beeinträchtigen.

Wie im vorigen $\$$ ergiebt das Product der Ecken

$$
u_{4}^{3} \cdot u_{2}^{3} \cdot u_{3}^{3}\left(u_{1}-u_{2}\right)=0 \text {, }
$$

für diese verhältnissmässig speciellere Lagen, als eine willkürliche Näherung dreier Ebenen bedingt. Die consecutiven Ebenen bestimmen für sich drei ebensolche Gerade $\left(x_{3}=x_{4}=0\right)$ und die einfache Ecke $\left(u_{1}-u_{2}=0\right)$. Der letztern entspricht die Kante der einfachen Ebenen. Die beiden dreifachen Punkte $u_{4}^{3}$ und $u_{3}^{3}$ sind demnach Durchschnitte der dreifachen Geraden mit letztgenarınten Ebenen, während deren Kante den Punkt $u_{1}^{3}$ auf der dreifachen Ebene verzeichnet. Auf Tafel II, Fig. 5 sind diese Verhältnisse zur Anschauung gebracht. -

Die Ebene $x_{1}+x_{2}=0$ ist hiernach als einzige Diagonalebene gekennzeichnet, es giebt daher Flächen mit zwei Knoten.

Aber im Allgemeinen haben die vorliegenden Flächen keine Singularitäten. Wir bemerken aber, dass der Durchschnitt mit der dreifachen Ebene $x_{3}=0: \frac{x_{1}{ }^{3}}{\alpha_{1}{ }^{2}}+\frac{x_{2}{ }^{3}}{\alpha_{2}{ }^{2}}=0$ aus drei sich in $x_{1}=x_{2}=x_{3}=0$ treffenden Geraden besteht. Folglich: Die dreifache Ebcne enthält stets drei Gerade der Fläche, welche sich im Schnittpunkte jener mit den beiden cinfachen Elenen treffen. Je nachdem die letztern reell oder conjugirt sind, erhält man eine oder drei reelle der bezeichneten Linien.

Das Verschwinden des Ausdrucks

deutet den Knoten

$$
-\frac{1}{2} \gamma \alpha_{3}^{3}+\alpha_{1}+\alpha_{2}
$$

$$
u_{1}, a_{2}, a_{3}, 0
$$

an. $A l_{s}$ zur Eintheilung verwerthbare Specialfiachen erweisen sich die Arten mit $\alpha_{\mathrm{i}}==\infty$ :

$$
\frac{x_{1}{ }^{3}}{\alpha_{1}{ }^{2}}+\frac{x_{2}{ }^{3}}{\alpha_{2}{ }^{2}}+\gamma x_{3}{ }^{3}-3 x_{3} x_{1}{ }^{2}=0 \text {. }
$$

Die linke Seite dieser Gleichung erscheint nach Reduction der binären cubischen Form $\gamma_{3}{ }^{3}-3 x_{3} x_{4}{ }^{2}$ auf zwei Cuben als Summe von vieren. Die beiden letcten sind uun reell oder conjugirt, je nachdem $\gamma \leqslant 0$, und wir haben also (vergl. $\$ \$ 2 ., 5 ., 6$.), wenn:
a) $x_{1}$ und $x_{2}$ reell und $\gamma<0$ eine $1 V^{\prime}$,
b) $x_{1} \quad, x_{n}, \quad " \gamma>0 \quad$ IV
c) $x_{1} " x_{2}$ conjugirt " $\gamma<0$ "IV",
d) $x_{1} \quad x_{2} \quad " \quad y>0$ " II.

Wir haben noch die frähere Bezeichnung beibebalten, obgleich für die unserigen Gleichungstormen in gewissen Fällen die Unterscheidung der verschiedenen Arten IV wegfällt. 
Die Fläche $\gamma=0$ besitzt die Eigenthümlichkeit, dass sie nie einen Knoten haben kann, soudern bei der Gleichbeit der Grössen $\alpha_{1}$ und $\alpha_{2}$ sofort zwei auftreten. Eine beliebige Aenderung von $\gamma$ muss also bei constanten $\alpha_{1}$ und $\alpha_{2}$ aus letzterer Fläche eine andere ohne Knoten erzeugen und zwar muss die Auflösung der Knoten bei beiden die nämliche gewesen sein, da keiner ausgezeichnet ist. Wir werden dieses Resultat bald verwerthen.

Zur Erzeugung von reellen Knoten sind, bei reellen Ebenen $x_{1}$ und $x_{2}$, auch reelle $\alpha_{1}$ und $\alpha_{2}$ erforderlich, welche wir zunächst voraussetzen.

Bei diesen Flächen ist die Ableitung der verschiedenen Arten nicht so einfach wie sonst, da es uns an einer Fläche fehlt, die genügend viele reelle Linien besitzt, um Aenderungen durch die biplanare Form an einem erzeugten Knoten ohne Einfluss zu lassen. Uns stehen bis jetzt nur Arten IV' und IV" zu Gebote. Unter Zugrundelegung eines allgemeinen Pentaeders könnten wir mit Sicherheit, von diesen Flächen ausgehend, beim ersten Erscheinen eines Knotens auf eine IV' resp. III' schliessen. Bei dem vorliegenden Pentaeder bedarf dieser Schluss einer Bestätigung. Sehr einfach ist diese bei der IV', da ein imarrinärer Kegel des Knotens die Fläche völlig charakterisirt. Ein solcher Kegel findet sich wirklich, wie man durch eine leichte Rechnung findet, bei der Fläche mit

$$
-\frac{1}{2} \gamma \alpha_{3}^{3}-\alpha_{1}-\alpha_{2}=0 \ldots \gamma<0,
$$

welche durch Eindlichwerden von $\alpha_{3}$ aus der unter a) angeführten IV' entsteht. Setzen wir insbesondere $\alpha_{1}=\alpha_{2}=a$, so geht diese Art auch aus der Fläche mit zwei Knoten: $\gamma=0, \alpha_{1}=\alpha_{2}=\alpha$ durch Uebergehen von $\gamma$ ins Negative hervor; wodurch letatere als III charakterisirt wird. Diese Aenderung von $\gamma$ bewirlt also an den beiden Knoten zunächst ein Trennen und dann ein Zusammenziehen des paaren Theils der jetzigen $\mathrm{V}$ zum isolirten Knoten. Nach einer oben gemachten Bemerkung muss daher ein Positivwerden von $\gamma$ ein Verbinden von Flächentheilen mit sich führen: die zunächst entstehenden Flächen sind III. Der bei weiterm Wachsen von $\gamma$ noch auftretende Knoten, wenn

$$
-\frac{1}{2} \gamma \cdot u_{3}^{3}+2 u=0,
$$

gehört jetzt nothwendig einer III' an, da diese Fläche ersichtlich nicht direct aus der III mit zwei Knoten durch Auflösung eines derselben hervorgehen kann, andererseits aber auch nicht vou einem grössern Zusammenhange ist, da ein Verschwinden des Knotens, hervorgerufen durch Wachsen von $\alpha_{3}$, zu einer Fläche führt, welche für $\alpha_{3}=\infty$ ạf continuirliche Weise in die IV" unter b) übergeleitet ist.

Die Untersuchung der Flächen mit beliebigem $\alpha_{1}$ und $\alpha_{2}$ ist von nun an leicht, da die begrenzenden Gebiete der Flächen mit Knoten 
gefunden sind. Wir verweisen des Nähern wegen auf Tabelle I. am Schlusse.

Bisher hatte die Aufstellung von Unterarten der IV also noch Sinn; sind jedoch beliebig viele der Grössen $\alpha_{1}$ und $\alpha_{2}$ imaginär, so ist der Unterschied verschwunden. Denn an den Specialflächen a) und b) hat sich offenbar gar nichts geändert und auch ein endliches $\alpha_{3}$ kann nie eine Abänderung der Art bewirken, da reelle Knoten unmöglich sind, wir können insbesondere $\gamma$ ungestört von positiven zu negativen Werthen fübren und so die $I V^{\prime}$ in eine $I V^{\prime \prime}$ verwandeln, womit dann die Gleichartigkeit dieser Flächen ausgesprochen ist. Ist bei gleichen $\alpha_{1}$ und $\alpha_{2}, \gamma=0$, so hat man die Fläche mit imaginären Knoten.

Bei conjugirten $x_{1}$ und $x_{2}$ ist durch die unter d) gefundene Fläche II ein günstiges Ausgangselement gegeben. Die auftretenden Flächen mit zwei Kuoten sind II', da eine geringe Deformation der dreifachen Ebene in drei getrennte nur die angegebene Art als möglich erscheinen lässt. (\$§ 5., 6.) Die Flächen mit einem Knoten sind daher II oder II' und das alleinige Auftreten letzterer von Arten III überhaupt beweist, dass die Bezeichnung der durch Trennen aus ihnen hervorgehenden Fläche ohne Knoten mit IV" noch Berechtigung hat. Imaginäre Knoten können wie sonst auftreten, sobald die conjugirten Ebenen reelle positive Coefficienten haben.

\section{Tabelle I.}

Reelle einfache Ebenen.

Fläche: $\frac{x_{1}{ }^{3}}{\alpha_{1}{ }^{2}}+\frac{x_{2}{ }^{3}}{\alpha_{2}{ }^{2}}+\gamma x_{3}{ }^{3}-3 \frac{x_{3}{ }^{2}\left(x_{1}+x_{2}\right)}{\alpha_{3}{ }^{2}}-3 x_{3} x_{4}{ }^{2}=0 \ldots \alpha_{1} \leq \alpha_{2}$.

a) $\alpha_{1}^{2}$ und $\alpha_{2}^{2}$ positiv.

$$
\gamma \geqq 0
$$

Variationen einer III: $-\frac{\gamma \alpha_{3}{ }^{3}}{2}\left\{\begin{array}{l}+\alpha_{1}+\alpha_{2} \cdots+\cdots \mathbf{A} \\ -\alpha_{1}+\alpha_{2} \cdots-\cdots \mathrm{B} .\end{array}\right.$

1) Elächen ohne Ḱnoten.

$\begin{array}{ll}\text { Zeichenwechsel: } & \text { Art: } \\ 0 & \text { III } \\ 1 \text { unter B } & \text { IV } \\ 1 \text { 1 A } & \text { IV" }\end{array}$

2) Flächen mit einem Knoten. Null unter B

3) Flächen mit zwei Knoten.

" " A III'

2 Nullen 


$$
\gamma<0 \text {. }
$$

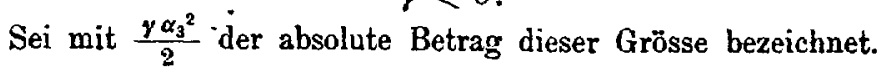

$$
\operatorname{tr} \gamma \alpha_{3}^{3}: \begin{cases}+\alpha_{1}-\alpha_{2}<0 & \text { Art: } \\ +\alpha_{1}-\alpha_{2}=0 & \text { IV mit einem Kuoten. } \\ +\alpha_{1}-\alpha_{2}>0 & \text { V } \\ -\alpha_{1}-\alpha_{2}=0 & \text { IV' mit einem Kroten. } \\ -\alpha_{1}-\alpha_{2}>0 & \text { IV'. }\end{cases}
$$

b) Eine oder beide der Grössen $\alpha_{1}^{2}$ und $\alpha_{2}^{2}$ negativ.

Alle Arten sind IV, aber die Eintheilung in Uuterarten fällt fort $\gamma=0, \alpha_{1}=\alpha_{2}$ liefert die Uebergangsflächen: Schläfli'sche IV, 5 .

\section{Tabelle II.}

Conjugirte einfache Ebenen.

Fläche: $\frac{\left(x_{1}+i x_{2}\right)^{3}}{\left(\alpha_{1}+i \alpha_{2}\right)^{2}}+\frac{\left(x_{1}-i x_{2}\right)^{3}}{\left(\alpha_{1}-i \alpha_{2}\right)^{2}}+\gamma x_{3}^{3}-6 \frac{x_{3}^{2} x_{1}}{\alpha_{3}^{2}}-3 x_{2} x_{4}^{2}=0$.

$-\frac{1}{2} \gamma \alpha_{3}{ }^{3}+2 \alpha_{1} \leqq 0\left\{\begin{array}{l}\text { II } \\ \text { Il mit Knoten; wernn } \gamma=0 \text { II' mit zwei Knoten. } \\ \text { III (oder Schläfli'sche IV, 6). }\end{array}\right.$

$$
\gamma>0 \text {. }
$$

$$
\gamma<0 \text {. }
$$

$-\frac{1}{2} \gamma \alpha_{3}^{3}-2 \alpha_{1} \geq 0\left\{\begin{array}{l}\text { IV }^{\prime \prime} \\ \text { III' mit Kuoten } \\ \text { III }\end{array}\right.$

\section{$\$ 14$.}

Die Flächen mit einer dreifachen und einer Doppelpentaederebene.

Aus der gefundenen Gleichung

$$
a x_{1}^{3}+b x_{1}^{2}\left(y_{1}+y_{2}\right)+c x_{1} y_{1}^{2}+d x_{2}^{3}+c x_{2}^{2} z_{1}=0
$$

leiten wir die einfachere

$$
3 x_{1}\left(\frac{x_{1}^{2}}{\alpha_{1}^{2}}-\frac{x_{2}^{2}}{\alpha_{2}^{2}}\right)+\gamma x_{1}{ }^{3}+\delta x_{2}{ }^{3}-3 x_{1} x_{3}{ }^{2}=0,
$$

in der $x_{2}=0$ die dreifache, $x_{2}=0$ die Doppelebene des Peutaeders ist. Die Constante $\delta$ wollen wir stets positiv wählen.

$$
x_{1}=x_{2}=x_{3}=0 \text { ist ein biplanarer Punkt mit den Ebenen }
$$

$$
\frac{x_{1}}{\alpha_{1}}+\frac{x_{2}}{\alpha_{2}}=0, \quad \frac{x_{1}}{\alpha_{1}}-\frac{x_{2}}{\alpha_{2}}=0,
$$


aber die Axe $\left(x_{1}=x_{2}=0\right)$ gehört jetzt der Fläche an, der Knoten ist ein $B_{4}$. Da ferner $x_{1}=0$ die Fläche längs der Axe berührt, so können wir den Satz hinstellen: Die Fläche besitzt einen $B_{4}$, dessen Ebenen harmonisch zur dreifachen und Doppelebene des Pentaeders sind; die dreifache Ebene ist die Tangentenebene längs der Axe.

Die Flächen sind die allgemeinsten mit der auftretenden Singularität. Während bei einem $B_{3}$ noch verschiedene Pentaeder möglich sind, bedingt ein $B_{4}$ stets eins der vorliegenden Art, da durch drei Elemente eines harmonischen Büschels (die Ebenen des Knotens und die Tangentenebene) stets das vierte eindeutig bestimmt ist.

Das Product der Ecken

$$
u_{4}^{6} \cdot u_{3}^{3} \cdot u_{2}=0
$$

giebt den $B_{4}$ als sechsfach zählend (die beiden dreifachen bei der vorigen Fläche haben sich durch das Zusammenrücken der damals getrennten Ebenen vereinigt). Die übrigen Ecken sind geblieben. Eine Diagonalebene: $x_{4}=0$, ist noch vorhanden, ein Zeichen, dass noch zwei conische Knoten auftreten können.

Die Fläche hat einen Knoten

wenn

$$
\alpha_{1}, \alpha_{2}, 0,-\gamma \alpha_{1}^{3}+\delta \alpha_{2}^{3},
$$

$$
\gamma \alpha_{1}^{3}+\delta \alpha_{2}^{3}=0 \text {. }
$$

Die weitere Untersuchung vereinfacht sich wesentlich, wenn man die Fälle reeller und conjugirter Ebenen des Knotens trennt. Danu können die $\alpha$ der Einheit gleich gesetzt werden, da sie nie unendlich werden dürfen. Mit dieser Erleichterung können wir dann unsere Flächen darstellen durch die Gleichungen

$$
3 x_{4}{ }^{2}\left(x_{t}{ }^{2} \mp x_{2}{ }^{3}\right)+y_{1}{ }^{3}+d x_{2}{ }^{3}-3 x_{1} x_{3}{ }^{2}=0,
$$

wo das obere Zeichen reelle, das untere conjugirte Ebenen des Knotens bedingt.

Aus der angegebenen Bedingung für den Knoten ist jedoch zu ersehen, dass ein solcher nur im ersten Falle reell sein kann, im zweiten existirt nur eine Art, da nie ein Uebergang zu einer andern stattfinden kann.

Bemerken wir, dass auf der Specialfläche $\delta=0, \gamma>0$ stets die beiden Linien reell sind, welche in jeder Ebene des Knotens ausser der Axe noch liegen, d. h. überhaupt alle Geraden reell sind, so finden sich leicht die Resultate, wie sie in der folgenden Tabelle zusammengestellt sind. 


\section{Tabelle I.}

Reelle Ebenen des Knotens.

$$
\begin{gathered}
3 x_{4}\left(x_{1}{ }^{2}-x_{2}{ }^{2}\right)+\gamma x_{1}{ }^{3}+\delta x_{2}{ }^{3}-3 x_{1} x_{3}{ }^{2}=0 \cdots \delta \geq 0 . \\
y \geq 0 .
\end{gathered}
$$

$$
\begin{aligned}
& \gamma \gtreqless \delta\left\{\begin{array}{lcc}
\text { Alles reell } & \text { Schläfli'sche V, } 1, \\
\text { In } x_{1}+x_{2}=0 \text { ein Knoten } & \text { X, für } \gamma=0 \text { zwei } \\
\text { Nur in } x_{1}-x_{2}=0 \text { zwei } & & \text { Knoten XVIII, } 1,
\end{array}\right. \\
& \text { reelle Strahlen } \\
& \gamma<0
\end{aligned}
$$

Wenn dem absoluten Werthe nach

$$
\gamma \leqq \delta \text {, erhält man }\left\{\begin{array}{cc}
\text { Schläfli'sche } \mathrm{V}, 2, \\
” & \mathrm{X}, 2, \\
" & \mathrm{~V}, 3 .
\end{array}\right.
$$

\section{Tabelle II.}

Conjugirte Ebeneu des Knotens.

$$
3 x_{4}\left(x_{1}{ }^{2}+x_{2}{ }^{2}\right)+\gamma x_{1}{ }^{3}+\delta x_{2}{ }^{3}-3 x_{1} x_{3}{ }^{2}=0 \text {. }
$$

Schläfli'sche V, 4 (oder XVIII, 2 mit zwei conjugirten Knoten).

\section{$\S 1 \overline{0}$.}

Die Flächen mit fünffacher Pentaederebene.

Bringt man die abgeleitete Gleichung

$$
a x_{1}{ }^{3}+b x_{1}{ }^{2}\left(y_{1}+y_{3}+y_{3}+y_{4}\right)+c x_{1}\left(y_{1}{ }^{2}+y_{1} y_{2}+y_{1} y_{3}+y_{2}{ }^{2}\right)
$$

auf die Form

$$
+d y_{1}^{3}+e y_{1}^{2} y_{2}=0
$$

$$
\begin{aligned}
a x_{1}^{3}+x_{1}{ }^{2}\left(a_{1} x_{1}+a_{2} x_{2}+a_{3} x_{3}+a_{1} x_{4}\right) & +c x_{1}\left(x_{2}{ }^{2}+x_{2} x_{3}+x_{2} x_{1}+x_{3}{ }^{2}\right) \\
& +d x_{2}{ }^{3}+e x_{2}{ }^{2} x_{3}=0,
\end{aligned}
$$

so erkennt man den biplanaren Knoten $x_{1}=x_{2}=x_{3}=0$ mit den Ebenen $x_{1}=0, a_{4} x_{1}+c x_{2}=0$ : Die Fläche hat einen $B_{n}$, alie fünfache Pentaederebene ist diejenige Ebene des Knotens, welche lüngs acr Axe berïhrt.

Durch das Zusammenfallen der beiden im rorigen $\$$ noch getrennt erscheinenden mehrfachen Pentaederebenen hat sich auch eine Ebene des Knotens mit jenen vereinigt, was als directe Folge der harmonischen Lage erklärlich ist.

Das Product der Ecken

$$
u_{4}^{10}=0
$$

liefert den $B_{5}$ als zehnfach zühlend und das Fehlen einer Diagonalebene 
beweist, dass höchstens ein Knoten auftreten kann. Die Gleichung der Fläche lässt sich reduciren auf

$$
x_{1} x_{2} x_{4}+3 x_{1} x_{3}{ }^{2}+3 x_{2}{ }^{2} x_{3}-a x_{1}{ }^{3}=0,
$$

welche für $a=0$ den als noch möglich hingestellten Knoten in der Ebene $x_{2}=0$ enthalten zeigt, dureh dessen verschiedenartige Anflösung die Arten der Tabelle 1. hervorgehen. -

Ein besonderer Fall tritt ein, wenn in der ursprünglichen Gleichung $e=0$ ist. Dann ist die Beseitigung des Gliedes $x_{2}{ }^{3}$ nicht mehr. möglich und die einfachiste Form ist

$$
x_{1} x_{2} x_{4}+3 x_{1} x_{3}{ }^{2}+3 x_{2}{ }^{3}-a x_{1}{ }^{3}=0 .
$$

Der biplanare Punlit ist jetzt ein $B_{6 j}$, die Pentaederebene osculirt. Das Product der Ecken verschwindet identisch. Man erhält auch für einen beliebigen Punkt der Axe

als Polare das Ebenenpaar

$$
0,0, y_{3}, y_{4}
$$

$$
x_{1}\left(x_{3} y_{3}+x_{2} y_{4}\right)=0 .
$$

Alle den Punkten jener Reihe entsprechenden Kanten bilden demnach einen ihr projectivische Büschel, dessen Centrum der Knotenpunkt ist. Hiernach ist die Fläche (vergl. $\$ 12$.) aus der mit $2 B_{3}$ durch Zusammenrücken letzterer abzuleiten, wobei dann auch deren Axen sich zur Axe des $B_{6}$ vereinigen.

Die Untersuchung der Fläche selbst ist wie die der vorigen. Wir verweisen auf die Tabelle, welche übrigens nur eine Reproduction der Schläfli'schen ist.

\section{Tabelle $\mathrm{I}$.}

Alle Ecken bestimmt: Flächen mit einem $B_{5}$.

In der nicht berührenden Ebene, je nachdem

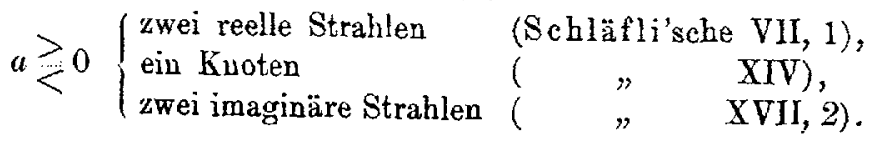

Tabelle 프.

Die Ecken theilweise unbestimmt: F'lächen mit einem $B_{6}$.

Das Verhalten der nicht osculirenden Ebene ist wie in Tabelle I. Die Fläche ist, wenn

$$
a \geq 0\left\{\begin{array}{cc}
\text { Schläfli'sche XI, } 1, \\
" & \text { XIX, } \\
" & \text { XI, }, .
\end{array}\right.
$$




\section{$\$ 16$.}

Die Flächen mit unbestimmten Pentaedern.

Es erübrigt noch die Flächen zu betrachten, bei denen nicht alleiu einige Ecken, soudern auch Ebenen unbestimmt sind. Conische Knoten, ein $B_{3}$ oder zwei $B_{3}$; sowie die übrigen Arten biplanarer Knoten geben hierzu im Allgemeinen noch keine Veranlassung. Aber wie wir schon wissen, giebt es andererseits Flächen ohne Singularitäten, welche eine ganz unbestimmte Pertaederebene haben; nämlich diejenigen, welche sich auf 4 Cuben reduciren lassen. Wir werden diese Arten unter den etwas allgemeinern finden, deren Pentaeder aus vier sich in einem Punkte treffenden Ebenen und einer beliebigen fünften besteht, wo dann erstere vier gewisse Unbestimmtheiten zeigen.

Ausser diesen sind zu untersuchen die Flächen mit uniplanarem Punkte und die Regelflächen. Erstere treten unter speciellen Annahmen von Coefficienten überall auf, sobald das Pentaeder mindestens eine mehrfache Ebene hat, wic sich leicht an den Gleichungen dieser Flächen ersehen lässt und wir überdies bei einigen Pentaedern erkannt haben, wo sich diese Flächen der Ableitung mancher Arten störend entgegenstellten. Es bleibt demnach zu zeigen, wie jene speciellen Formen in einer allgemeinern enthalten sind.

Regelflächen besitzen gewissermassen in jedem Punkte ibrer Doppelgeraden einen biplanaren Punkt und in zweien derselben uniplanare, welche sich auch noch vereinigen können. Das Pentaeder wird daher solche Willkürlichkeiten in der Gestalt aufweisen, dass man seine Ebenen, ohne die Fläche zu ändern, in Lagen bringen kann, wie sie die angegebenen Singularitäten bedingen.

\section{$\S 17$.}

Die Flächen, deren Pentaeder vier Ebenen durch einen Punkt besitzt*). (Flächen mit einem osculirenden Kegel.)

Dieser Specialisirung des Pentaeders entspricht die Gleichung

$$
\begin{gathered}
\frac{x_{1}^{3}}{\alpha_{1}{ }^{2}}+\frac{x_{2}^{3}}{x_{2}{ }^{2}}+\frac{x_{3}{ }^{3}}{\alpha_{3}{ }^{2}}+\frac{x_{4}{ }^{3}}{\alpha_{4}{ }^{2}}+\frac{x_{3}^{3}}{\alpha_{3}{ }^{2}}=0 . \\
x_{1}+x_{4}+x_{3}+x_{4}=0 .
\end{gathered}
$$

Vom Schnittpunkt der vier ausgezeichneten Ebenen geht ein osculirender Kegel an die Fläche, dessen Berïhrungscurve in der fünften liegt und durch

*) Vergl. Eckardt a. a. $0 . \$ 8$. 


$$
\frac{x_{1}^{3}}{\alpha_{1}^{2}}+\frac{x_{2}{ }^{3}}{\alpha_{2}^{2}}+\frac{x_{3}^{3}}{\alpha_{3}^{2}}+\frac{x_{4}{ }^{3}}{\alpha_{4}{ }^{2}}=0, \quad x_{5}=0
$$

gegeben ist. Jede ternäre cubische Form lässt sich aber auf zweifach unendlich viele Arten auf vier Cuben reduciren, mithin unsere Gleichung eben so oft auf fünf, und die Frage nach der kanonischen Form ist, sobald die Ebene $x_{5}=0$ gefunden, übereinstimmend mit der nämlichen für Curven.

Wie Eckardt bemerkt hat, schneiden sich die 27 Geraden der Fläche neunmal zu dreien in den Wendepunkten der.Berührungscurve, während die 9 Ebenen, deren jede solche drei Linien ausschneidet, durch die Spitze des Kegels gehen. Man erkennt dieses leicht durch Herstellung der Form

$$
x_{1} x_{2} x_{3}+k\left(x_{1}+x_{2}+x_{3}\right)^{3}+\frac{x_{5}^{3}}{\alpha_{5}^{2}}=0,
$$

welche nach bekannten Sätzen über Curven dritter Ordnung eine allgemeine ist*). Die Ebenen $x_{1} x_{2} x_{3}$ schneiden die Geraden aus, welche sich in den drei reellen Wendepunkten (auf $x_{1}+x_{2}+x_{3}=0$ ) treffen. In jeder Ebene ist stets nur eine reell. Die übrigen 18 sind aber sicher imaginär, da es ihre Kreuzungspunkte - die Wendepunkte - sind. Demnach sind nur drei Gerade der Fläche reell. Es kann jedoch nie die Art V auftreten. Denu, sei der Theil.des Berührungskegels, welcher dem paaren Stück der Fläche angehört, reell, so schneidet jede seiner Erzeugenden den unpaaren auch noch, osculirt also nicht. Ist andererseits jener Theil imaginär, so wird das paare Stiick von jeder Geraden durch den Scheitel geschnitten, es kann also ebenfalls keine osculiren. Folglich: Geht von einem Eckpunkte des Pentaeders ein osculirender Kegel an die Fläche, so ist dieselbe im Allgemeinen eine der Arten IV, welche jedoch jetzt in einander verschmolzen sind.

$\mathrm{Ob}$ Knoteu vorbanden sind oder nicht, hängt einzig und allein von der Berührungscurve, nicht etwa noch von dem Coefficienten von $\dot{x}_{5}{ }^{3}$ ab. Besitzt jene Curve einen Doppelpunkt, so ist dieser auch Kuoten der Fläche, wie man der Gleichung sofort ansieht. Sofern nämlich der Doppelpunkt ein gewöhnlicher ist, kann die Form

sofern er ein Rückkehrpunkt ist, die Form

$$
x_{1} x_{2} x_{3}+f_{3}\left(x_{1} x_{2}\right)+\frac{x_{5}^{3}}{\alpha_{3}^{2}}=0
$$

$$
x_{1}{ }^{2} x_{3}+f_{3}\left(x_{1} x_{2}\right)+\frac{x_{3}{ }^{3}}{\alpha_{5}^{2}}=0,
$$

hergestellt werden und diese Gleichungen lassen einen biplanaren resp.

*) Die Flächen, bei denen sich die oben angeweudete Form nicht berstellen lïsst, finden im nächsten $\S$ ihre Erledigung, mit ihmen auch solche mit conjugirt
imaginären osculirenden Kegeln. 
einen uniplanareu Punkt erkennen; conische Knoten treten daher überhaupt nicht auf.

Die letatgenannte Fläche kann aber nicht mehr auf fünf Cuben gebracht werden, dann ihre Berührungscurve nicht mehr auf vier. Wir betrachten sie daher, als nicht unserem Pentaeder angehörig, später (\$ 18.).

Bei der Annahme eines òder zweier weiterer Doppelpunkte, welcher die Ausartung der Curve in einen Kegelschnitt und eine Grerade resp. in drei Gerade entspricht, erhalten wir ebenso viele neue biplanare Knoten. Aber von allen erzeugten Flächen ist nur die letzte mit dreien die allgemeinste ihrer Art hinsichtlich der auftretenden singulären Punkte; denn nach Schläfli kann solche Fläche dargestellt werden durch die Gleichung

$$
x_{1} x_{2} x_{3}+x_{5}{ }^{3}=0
$$

welche die Ausartung des osculirenden Kegels in drei Ebenen $x_{1} x_{2} x_{3}$ zeigt. Damit sind alle Fälle aufgezählt.

\section{Tetraedertlächen.}

Wir behandeln jetzt einen Specialfall des vorigen $\$$, dessen wir dort in einer Note erwähnten. Verschwindet für die Berührungscurve des Kegels die Aronhold'sche Invariante $S$, so schneiden sich ihre Wendetangenten dreimal zu dreien in einem Punkte und die Form, deren wir uns zur Untersuchung der Realität der Geraden auf der Fläche bedienten, lässt sich nicht mehr herstellen. Bekanntlich sind aber unter dieser Bedingung nur drei Cuben zur Darstellung der Curve erforderlich, die Transformation ist eine völlig bestimmte und ebeuso also auch diejenige unserer Flächen auf vier Cuben. Da hiernach den vorliegenden Gattungen nur noch ein Tetraeder zugeordnet ist, haben wir sie mit obigem Namen belegt.

Aus der Gleichberechtigungr aller vier Ecken des Tetraeders folgt: Verschwindet für die Beriührungscurve des eine Fläche osculirenden Kegels die Aronhold'sche Invariante $S$, so sind vier solche Kegel vorhanden. Nimmt man die Elenen der Curven zu Coordinatenebenen, so erscheint die Gleichung der Fläche als Aggregat von vier Cuben.

Hierbei ist vorausgesetzt, dass nicht etwa zwei oder mehr Ebenen vereinigt liegen, welche Fälle wir, der völligen Bestimmtheit jener Elemente wegen, von nun an wieder besonders zu betrachten haben.

Berücksichtigen wir noch Verschiedenheiten, welche hinsichtlich der Realität der Ebenen sich darbieten, so finden wir nach $\$ 9$. in Ganzen 9 Arten, die folgenden: 
a) Eigentliches Tetraeder.

1) $x_{1}{ }^{3}+x_{2}{ }^{3}+x_{3}{ }^{3}+x_{4}{ }^{3}=0 \ldots \ldots \ldots \ldots \ldots V^{\prime}(\S 4$.$) ,$

2) $x_{1}{ }^{3}+x_{2}{ }^{3}+\left(x_{3}+i x_{4}\right)^{3}+\left(x_{3}-i x_{4}\right)^{3}=0 \cdots \cdots$ IV $^{\prime \prime}(\S 5$. $)$,

3) $\left(x_{1}+i x_{2}\right)^{3}+\left(x_{1}-i x_{2}\right)^{3}+\left(x_{3}+i x_{4}\right)^{3}+\left(x_{3}-i x_{4}\right)^{3}$. . II (\$6.).

b) Eine Doppelebene $x_{3}=0$.

4) $x_{1}{ }^{3}+x_{2}{ }^{3}+x_{3}{ }^{2} x_{4}=0 \ldots$ Fläche mit $U_{6}$ (Schläfli'sche XII, 2),

5) $\left(x_{1}+i x_{2}\right)^{3}+\left(x_{2}-i x_{2}\right)^{3}+x_{3}^{2} x_{4}=0 \ldots$ Fläche mit $D_{6}$ (Schlä fli'. sche XII, 1).

c) Zwei Doppelebenen $x_{1}=0, x_{3}=0$ resp. $x_{1}+i x_{3}=0, x_{1}-i x_{3}=0$.

6) $x_{1}{ }^{2} x_{2}+x_{3}{ }^{2} x_{4}=0 \ldots$ Regelfläche (Schläfli'sche XXII, 1),

7) $\left(x_{1}+i x_{3}\right)^{2} x_{2}+\left(x_{1}-i x_{3}\right)^{2} x_{4}-0 \quad " \quad$, $\quad$ XXII,2).

d) Eirue dreifache Ebene $x_{1}=0$, eine einfache Ebene $x_{2}=0$.

8) $x_{1}{ }^{2} x_{4}+x_{1} x_{3}{ }^{2}+x_{2}{ }^{3}=0 \ldots$ Fläche mit $U_{8}$ (Schläfli'sche XX).

e) Eine vierfache Ebene $x_{1}=0$.

9) $x_{1}{ }^{2} x_{4}+x_{1} x_{2} x_{3}+x_{2}{ }^{3}=0 \ldots$ Regelfläche mit vereinigten Directrixen (Cayley'sche).

Dic Hesse'sche Fläche fült bei jeder dicser Flächen mit dem ihur eigentliümlichen Tetraeder zusammen, aber nicht umgekehrt gehört eine Fläche, deren Hesse'sche aus Ebenen besteht, zu den vorliegenden. So z. B. kommt diese Eigenschaft den Flächen mit drei biplanaren Knoten zu, welche nichtsdestoweniger nur durch fünf Cuben darstellbar sind. -

Jede Ebene des Raumes bann als fünfte eines Pentaeders unserer Flächen betrachtet werden, indem man sie mit Null nultiplicirt deren Gleichung hinzufügt. Alle. Punkte der Kanten des Tetraeders sirıd daher als Ecken, alle Geraden der Seitenflüchen als Kanten des Pentaeders aufzufassen, was sich durch Polareubildung leicht bestätigt.

Dic Arten 1) mit vier reellen Kegeln und 2) mit zwei reellen Kegeln sind schon unter den Flächen des vorigen $\S$ enthalten; man braucht zu ihrer Erzeugung nur einen der Coefticienten $\alpha_{1,2,3,4}$ unendlich werden zu lassen. Hingegen die Art 3) mit vier imaginären Kegeln findet sich nicht vor, da überhaupt nur von reellen Flächen die Rede ist, alle angrenzenden Flächen mit nur einem osculirenden Kegel aber imaginär sind. Diese Fläche nimmt daher einen völlig isolirten Stand ein, was auch aus der abweichenden Zahl ihrer reellen Linien, 15 statt 3 , ersichtlich ist.

Bei den Flächen mit uniplanarem Punkte $U_{6}$ sind noch zwei eigent. liche osculirende Kegel vorhanden, die beiden übrigen sind vereinigt. in der Ebene des Kuotens. Bei 4) sind jene Kegel reell, bei 5) con- 
jugirt; ihre Berührungscurven haben an der singulären Stelle einen Rückkehrpunkt.

Während bei den bisherigen Arten das Pentaeder speciellerer Natur ist, als es die auftretende Singularität nothwendig macht, finden wir bei den Flächen $6-9$ stets die angegebene Gruppirung der Ebenen; die von uns gefundenen Gleichungen stimmen mit den Schläfli'schen überein. Nur bei 8 ) giebt es noch einen eigentlichen osculirenden Kegel, dessen Berührungscurve aus einem Kegelschnitt und einer ihn berührenden Geraden besteht.

Für die Regelflächen 6) tritt jetzt das Verhältniss zu denen mit $B_{3}$ hervor, dessen wir in $\$ 16$. erwähnten, da jeder Punkt der Doppeldirectrix $x_{1}=x_{3}=0$ als Schnittpunkt der beiden Doppelebenen des Pentaeders mit der beliehigen fünften angesehen werden kann, ein solcher Punkt aber nach $\S 11$. stets ein $B_{3}$ ist.

Ebenso kann man Eigenschaften, welche bei bestimmtem Pentaeder nur einem Punkte der Fläche zukommen, bei den übrigen der unserigen für unendlich viele nachweisen, was wir nicht weiter ausführen.

\section{$\S 19$.}

Die Flächen mit uniplanarem Punkte im Allgemeinen.

Nimmt man die Ebene des Knotens zu

$$
x_{1}+x_{2}+x_{3}=0 \text {, }
$$

so sind die vorliegenden Flächen enthalten in der Gleichung

$$
3 x_{4}\left(x_{1}+x_{2}+x_{3}\right)^{2}+f_{3}\left(x_{1}, x_{2}, x_{3}\right)=0 \text {. }
$$

Wählt man $x_{4}=0$ so, dass für ihre Schnittcurve mit der Fläche:

$$
f_{3}\left(x_{1}, x_{2}, x_{3}\right)=0
$$

die Invariante $S$ verschwindet, so lässt sich deren Gleichung durch drei Cuben darstellen, .so dass unter dieser Voraussetzung die Gleichung der Fläche die Form

$$
3\left(x_{1}+x_{2}+x_{3}\right)^{2} x_{4}+\frac{x_{1}^{3}}{\alpha_{1}^{2}}+\frac{x_{2}{ }^{3}}{\alpha_{2}^{2}}+\frac{x_{3}^{3}}{\alpha_{3}^{2}}=0
$$

aunehmen kann, welche wir benutzen werden. Es giebt unendlich viele solche Ebenen $x_{4}=0$, sie umhüllen eine Fläche vierter Classe, von Gordan*) Arouhold'sche Fläche genannt. Die angegebene Reduction ist demnach auf unendlich viele Arten ausführbar.

Die gefundene Form hat die grösste Aehnlichkeit mit derjenigen der Flächen mit Doppelpentaederebene, sie geht aus letzterer hervor,

*) Vergl. Gordan a. a. $0 . \$ 14$. 
wenn man die Doppelebene durch den Schnitt der drei einfachen legt. Folglich: Eine Flüche mit uniplanarem Punlte hat dessen Ebene zur Doppelebene des Pentaeders, während sich die drei einfachen in ithm schneiden, und umgekehrt. Die einfachen Ebenen sind dann nicht mehr völlig bestimmt.

Es handelt sich bei gegebener Fläche darum, die Bedingung zu finden, welcher drei durch den Knoten gehende Ebenen genügen müssen, um Pentaederebenen zu sein. Hierzu und zugleich zur Auffinlung des Orts der ehemaligen Ecken - unendlich viele sind es jedenfalls bilden wir die Hesse'sche Form:

$$
H=\left|\begin{array}{cccc}
\frac{x_{1}}{\alpha_{1}^{2}}+x_{4} & x_{4} & x_{4} & x_{1}+x_{2}+x_{3} \\
x_{4} & \frac{x_{2}}{\alpha_{2}^{2}}+x_{4} & x_{4} & x_{1}+x_{2}+x_{3} \\
x_{4} & x_{4} & \frac{x_{3}}{\alpha_{3}^{2}}+x_{4} & x_{1}+x_{2}+x_{3} \\
x_{1}+x_{2}+x_{3} & x_{1}+x_{2}+x_{3} & x_{1}+x_{2}+x_{3} & 0
\end{array}\right|=0
$$

oder ausgerechnet:

$$
\left(x_{1}+x_{2}+x_{3}\right)^{2}\left(\frac{x_{1} x_{2}}{\alpha_{1}^{2} \alpha_{2}^{2}}+\frac{x_{3} x_{3}}{\alpha_{2}^{2} \alpha_{3}^{2}}+\frac{x_{3} x_{1}}{\alpha_{3}^{2} \alpha_{1}^{2}}\right)=0 .
$$

Die Hesse'sche Fläche zerfällt also in einen Kegel zweiter Ordnung $K$ mit dem Knoten als Spitze und der zweifach zählenden singulären Ebene. Alle Unterdeterminanten $H_{i k}$ mit Ausnahme von

$$
H_{44}=\frac{x_{1} c_{2} x_{3}}{\alpha_{1}^{2} \alpha_{2}^{2} \alpha_{3}^{2}}+x_{4}\left(\frac{x_{1} x_{2}}{\alpha_{1}^{2} \alpha_{2}^{2}}+\frac{x_{2} x_{3}}{\alpha_{2}^{2} \alpha_{3}^{2}}+\frac{x_{3} x_{1}}{\alpha_{3}^{2} \alpha_{1}^{2}}\right)
$$

verschwinden für einen beliebigen Punkt ron $x_{1}+x_{2}+x_{3}=0 . \quad H_{44}=0$, $x_{1}+x_{2}+x_{3}=0$ ist demnach der gesuchte Ort der Ecken.

Die Pentaedereckan bilden folglich eine Curve aritter Ordnung $C$ in der Ebene des Knotens. Letzterer ist Doppelpunkt, dessen Tangenten die Schnittlinien des zur Hesse'schen Fläche gehörigen Kegels mit der Ebene der Curve sind.

Die Curve $C$ hat drei Wendepunkte auf einer Geraden. Demnach können wir unsere Gleichung noch dadurch vereinfachen, dass wir die Ebene $x_{1}=0$ jene Gerade verzeichnen lassen, wodurch die Coefficienten $\alpha$ specielle Werthe annehmen werden, aber sonst keine Aenderung der Form erfolgen wird.

Zur Auffindung dieser besondern Lage machen wir von dem Satze Gebrauch, dass bei einer Curve dritter Ordnung mit Doppelpunkt die Tangenten in ihm die Hesse'sche Form der durch seine Verbindungslinien mit den Wendepunkten gegebenen binären cubischen form darstellen. Also soll 


$$
\frac{x_{1} x_{2}}{\alpha_{1}^{2} \alpha_{2}^{2}}+\frac{x_{2} x_{3}}{\alpha_{2}^{2} \alpha_{3}{ }^{2}}+\frac{x_{3} x_{1}}{\alpha_{3}^{2} \alpha_{1}^{2}}=0, \quad x_{1}+x_{2}+x_{3}=0
$$

die Hesse'sche Form von

$$
\frac{x_{1}}{\alpha_{1}^{2} \alpha_{2}^{2} \alpha_{3} x_{3}{ }^{2}}=0
$$

sein; was nur der Fall ist, wenn $\alpha_{1}=\alpha_{2}=\alpha_{3}$. Nehmen wir noch diesen Werth der Einheit gleich, was der Allgemeinheit keinen $\mathrm{Ab}$ bruch thut, so entsteht die einfachste form:

$$
3 x_{1}\left(x_{1}+x_{2}+x_{3}\right)^{2}+x_{1}{ }^{3}+x_{2}{ }^{3}+x_{3}{ }^{3}=0 .
$$

Durch die Transformation

$$
x_{4}-\frac{1}{3}\left(x_{1}+x_{2}+x_{3}\right)=x_{1}^{\prime}, \quad x_{1}+x_{2}=x_{3}^{\prime} \text { etc. }
$$

erhalten wir Schläfli's Gleichung:

$$
\frac{3 x_{1}^{\prime}}{2}\left(x_{1}^{\prime}+x_{2}^{\prime}+x_{3}^{\prime}\right)^{2}-x_{1}^{\prime} x_{2}^{\prime} x_{3}^{\prime}=0 \text {, }
$$

welche ein übersichtliches Bild der Vertheilung der Geraden gewährt. Sie lässt die Wendepunkitę der Curve $C$ als Schnittpunkte der drei unären Geraden der Fläche mit denen des Knotens erkennen.

Nur so lange die Wendepunkte reell sind, sind es die Ebenen $x_{1}=x_{2}=x_{3}=0$, sofern man die specielle Lage der Ebenen $x_{4}$ verwendet, während im allgemeinen Falle immer alle drei reell sein können, da $\operatorname{man} x_{i}=0$ stets so legen kann, dass $C$ von ihr in drei reellen Punkten getroffen wird.

Zur Aufsuchung der zu einer als gegeben angenommenen Ebene $x_{4}=0$ gehörigen Pentaederebenen bemerken wir, dass jene offenbar die Rolle der Ebene $y_{1}$ bei den allgemeinsten Flächen mit Doppelebene spielt. (\$ 10.) Demnach ist ihr Scbnitt mit $x_{1}+x_{2}+x_{3}=0$ Pentaederkante, zu der also jede Gerade dieser Ebene gemacht werden kann. Die anf der Kante liegenden Ecker bestimmen sich als Schnitt mit $C$, und die einem solchen Punkttripel angehörigen Ebenen kann man etwa durch Polarenbildung finden, da jede derselben zwei der Doppelgeraden dieser Flächen enthalten muss. Sehr einfach erhält man die Pentaederebenen auch mit Hülfe der bereits in einer Note des $\$ 10$. benutzten Gordan'schen Formeln. Wir ziehen jedoch hier den andern Weg vor, da doch noch die Gleichung der Polaren später benutzt werden wird. Zu ihrer Bildung empfiehlt es sich, die Coordinaten von $C^{\prime}$ als rationale Functionen eines Parameters darzustellen, welches möglich, da die Curve vom Geschlecht $p=0$ ist. Es ist dann nach der gefundenen Gleichung:

wo

$$
x_{1}=\lambda_{1} m, \quad x_{2}=\lambda_{2} m, \quad x_{3}=\lambda_{3} m, \quad x_{4}=-\lambda_{1} \lambda_{2} \lambda_{3},
$$

$$
m=\alpha_{3}^{2} \lambda_{1} \lambda_{2}+\alpha_{1}^{2} \lambda_{2} \lambda_{3}+\alpha_{2}^{2} \lambda_{3} \lambda_{1}, \quad \lambda_{1}+\lambda_{2}+\lambda_{3}=0 \text {. }
$$


Die Gleichung der Polare eines beliebigen Punktes $y_{1} y_{2} y_{3} y_{4}$ :

$$
\begin{aligned}
y_{1}\left(x_{4}\left(x_{1}+x_{2}+x_{3}\right)+\frac{x_{3}^{2}}{\alpha_{1}^{2}}\right) & +y_{2}\left(x_{1}\left(x_{1}+x_{2}+x_{3}\right)+\frac{x_{2}^{2}}{\alpha_{2}^{2}}\right) \\
& +y_{3}\left(x_{4}\left(x_{1}+x_{2}+x_{3}\right)+\frac{x_{3}^{2}}{\alpha_{s}^{2}}\right)+y_{4}\left(x_{1}+x_{2}+x_{3}\right)^{2}=0
\end{aligned}
$$

geht für diese Werthe der $y$ über in:

$$
\begin{gathered}
\frac{x_{1}{ }^{2}}{\alpha_{1}{ }^{2}} \lambda_{1}{ }^{2}\left(-\frac{\lambda_{2}}{\alpha_{2}{ }^{2}}+\frac{\lambda_{3}}{\alpha_{3}{ }^{2}}\right)+\frac{x_{2}{ }^{2}}{\alpha_{2}{ }^{2}} \lambda_{2}{ }^{2}\left(\frac{\lambda_{3}}{\alpha_{3}{ }^{2}}+\frac{\lambda_{1}}{\alpha_{1}{ }^{2}}\right)+\frac{x_{3}{ }^{2}}{\alpha_{3}{ }^{2}} \lambda_{3}{ }^{2}\left(\frac{\lambda_{1}}{\alpha_{1}{ }^{2}}+\frac{\lambda_{2}}{\alpha_{2}{ }^{2}}\right) \\
-2\left(x_{1} x_{2}+x_{2} x_{3}+x_{3} x_{1}\right) \frac{\lambda_{1} \lambda_{2} \lambda_{3}}{\alpha_{1}{ }^{2} \alpha_{2}{ }^{2} \alpha_{3}{ }^{2}}=0,
\end{gathered}
$$

welche mit Nothwendigkeit ein Ebenenpaar darstellt.

Den drei Punkten auf $x_{4}=0$ entsprechen die Parameter

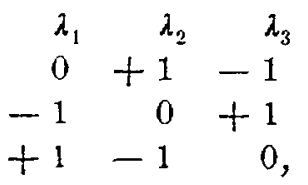

ibre Polaren sind demnach:

$$
\frac{x_{2}{ }^{2}}{\alpha_{2}^{2}}-\frac{x_{3}{ }^{2}}{\alpha_{3}{ }^{2}}=0, \quad \frac{x_{3}{ }^{2}}{\alpha_{3}{ }^{2}}-\frac{x_{1}{ }^{2}}{\alpha_{1}{ }^{2}}=0, \quad \frac{x_{1}^{2}}{\alpha_{1}^{2}}-\frac{x_{2}{ }^{2}}{\alpha_{2}{ }^{2}}=0
$$

Ebenenpare, deren Doppelgerade die Pentaederebenen

$$
x_{1}=0, \quad x_{2}=0, \quad x_{3}=0
$$

bestinumen und wie bei allgemeinen Flächen mit ihnen harmonische Büschel bilden. Hiermit ist bewiesen: Sobald eine Gerade der Ebene des Knotens als Pentaederkante angenommen ist, sind alle übrigen Elemente eindeutig bestimmt.

Es sei noch bemerkt, dass die Doppelgeraden der Polaren den Kegel $K$ bilden, nur dem Knoten entspricht doppeltzäblend seine Ebene als Polare, wodurch es sich erklärt, dass jede Gerade als Kante aufgefasst werden kann.

Die speciellen Formen, in denen wir früher den Gleichungen der vorliegenden Flächen begegneten, lassen sich jetzt leicht durch specielle Annahme der Kante in $x_{i}=0$ erklären, für welche zwei oder drei Pentaederecken auf ihr vereinigt liegen. -

Die Ebenenpaare der cerfallenden Polare umbüllen einen Kegel, zu dessen Untersuchung wir uns jetzt wenden wollen. Seine Gleichung dürfte folgendermassen am einfachsten aufzustellen sein. Ist $u_{x} v_{x}=0$ eines jener Ebenenpaare, so hat man identisch:

$$
\begin{aligned}
& \left(u_{1} x_{1}+u_{2} x_{2}+u_{3} x_{3}\right)\left(v_{1} x_{2}+v_{2} x_{2}+v_{3} x_{3}\right) \\
& =\frac{x_{1}{ }^{2}}{\alpha_{1}{ }^{2}} \lambda_{1}{ }^{2}\left(\frac{\lambda_{2}}{\alpha_{2}{ }^{2}}+\frac{\lambda_{3}}{\alpha_{3}{ }^{2}}\right)+\frac{\alpha_{2}{ }^{2}}{\alpha_{2}{ }^{2}} \lambda_{2}{ }^{4}\left(\frac{\lambda_{3}}{\alpha_{3}{ }^{2}}+\frac{\lambda_{1}}{\alpha_{1}{ }^{2}}\right)+\frac{x_{3}{ }^{2}}{\alpha_{3}{ }^{4}} \lambda_{3}{ }^{2}\left(\frac{\lambda_{1}}{\alpha_{1}{ }^{2}}+\frac{\lambda_{2}}{\alpha_{2}{ }^{2}}\right) \\
& -2\left(x_{1} x_{2}+x_{2} x_{3}+x_{3} x_{1}\right) \frac{\lambda_{1} \lambda_{2} \lambda_{3}}{\alpha_{1}^{2} \alpha_{2}^{2} \alpha_{3}^{2}} \text {. }
\end{aligned}
$$


Mit der abkürzenden Bezeichnung

$$
\frac{\lambda_{1}^{2}}{\alpha_{1}^{2}}\left(\frac{\lambda_{2}}{\alpha_{2}^{2}}+\frac{\lambda_{3}}{\alpha_{3}^{2}}\right)=p_{1} \text { etc. }
$$

findet man durch Vergleichung der Coefficienten der $x$

$$
\begin{aligned}
& \text { 1) } u_{1} v_{1}-\frac{p_{1}}{\alpha_{1}^{2}}=0, \\
& \text { 2) } u_{2} v_{2}-\frac{p_{2}}{\alpha_{2}^{2}}=0, \\
& \text { 3) } u_{3} v_{3}-\frac{p_{3}}{\alpha_{3}^{2}}=0, \\
& \text { 1) } u_{1} v_{2}+v_{1} u_{2}+2 \frac{\lambda_{1} \lambda_{2} \lambda_{3}}{\alpha_{1}^{2} \alpha_{2}^{2} \alpha_{3}^{2}}=0 . \\
& \text { 5) } u_{2} v_{3}+v_{3} u_{2}+2 \frac{\lambda_{1} \lambda_{2} \lambda_{3}}{\alpha_{1}^{2} \alpha_{2}^{2} \alpha_{3}^{2}}=0, \\
& \text { 6) } u_{3} v_{1}+v_{1} u_{3}+2 \frac{\lambda_{1} \lambda_{2} \lambda_{3}}{\alpha_{1}^{2} \alpha_{2}^{2} \alpha_{3}^{2}}=0 .
\end{aligned}
$$

Nimmt man hierzu noch $\lambda_{1}+\lambda_{2}+\lambda_{3}=0$, welche Gleichung sich auch ersetzen lässt durch

$$
\text { 7) } p_{1}+p_{2}+p_{3}+\lambda_{1} \lambda_{2} \lambda_{3}\left(\frac{1}{\alpha_{2}^{2} \alpha_{3}^{2}}+\frac{1}{\alpha_{3}^{2} \alpha_{1}^{2}}+\frac{1}{\alpha_{1}^{2} \alpha_{2}^{2}}\right)=0 \text {, }
$$

so hat man 7 Gleichungen, linear und homogen in den 7 Unbekannten

$$
v_{1}, v_{2}, v_{3}, p_{1}, p_{2}, p_{3}, \lambda_{1} \lambda_{2} \lambda_{3} \text {, }
$$

deren Elimination auf die Gleichung des gesuchten Kegels in Ebenencoordinaten:

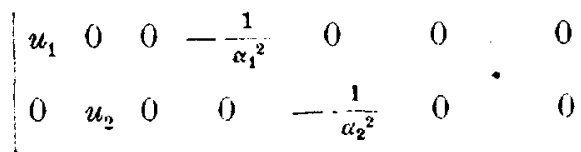

$$
\begin{aligned}
& \begin{array}{lllllll}
0 & 0 & u_{3} & 0 & 0 & -\frac{1}{\alpha_{3}^{2}} & 0
\end{array} \\
& P=\begin{array}{lllllll}
u_{2} & u_{1} & 0 & 0 & 0 & 0 & \frac{2}{\alpha_{1}^{2} \alpha_{2}{ }^{2} \alpha_{3}{ }^{2}}
\end{array} \\
& \begin{array}{lllllll}
0 & u_{3} & u_{2} & 0 & 0 & 0 & \frac{2}{\alpha_{1}^{2} \alpha_{2}^{2} \alpha_{3}{ }^{2}}
\end{array} \\
& \begin{array}{lllllll}
u_{3} & 0 & u_{1} & 0 & 0 & 0 & \frac{2}{\alpha_{1}^{2} \alpha_{2}^{2} \alpha_{3}^{2}}
\end{array}
\end{aligned}
$$

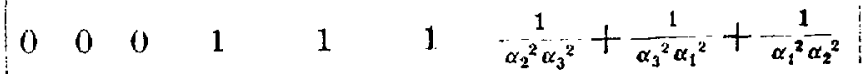

oder

$$
\begin{aligned}
& P=u_{1} u_{2} u_{3}\left(\alpha_{1}^{2}+\alpha_{2}^{2}+\alpha_{3}^{2}\right)+\alpha_{1}^{2} u_{1}^{3}+\alpha_{2}^{2} u_{2}^{3}+\alpha_{3}^{2} u_{3}^{3} \\
& -\alpha_{1}^{2} u_{1}^{2}\left(u_{2}+u_{3}\right)-\alpha_{2}^{2} u_{2}^{2}\left(u_{3}+u_{1}\right)-\alpha_{3}^{2} u_{3}^{2}\left(u_{1}+u_{2}\right)=0
\end{aligned}
$$

führt, zu der wir noch $u_{4}=0$ gesetzt denken müssen. 
Dieser Kegel ist dritter Classe und besitzt die Ebene des Knotens zur Doppeltangentenebene längs den Tangenten des Doppelpunkts der Curve $C$. Der Kegel $K$ wird dreimal von ihm berührt, längs den Doppelgeraden der Polaren, welche den drei Wendepunkten von C angehören, während die auftretenden drei Rückkehrtangentenebenen von $P$ selbst Polaren der nämlichen Punkte in Bezug auf $K$ sind. Diese Sätze sind einfach zu beweisen, wenn man die abgeleitete Gleichung anwendet, welche der Wendepunktslinie von $C$, als Pentaederkante gewählt, zukommt.

Fig. 1, Tafel $M I$ bringt die auftretenden Gebilde für den Fall eines $V_{8}$ mit drei reellen Geraden zur Anschauung. Der Knoten von $C$ ist isolirt, die Linie ihrer drei (reellen) Wendepunkte ist zur unendlich fernen gewählt.

\section{$\S 20$.}

Classification der Flächen mit nniplanarem Punkte.

Die Eläche

$$
x_{4}\left(x_{1}+x_{2}+x_{3}\right)^{2}+\frac{x_{1}{ }^{3}}{\alpha_{2}{ }^{2}}+\frac{x_{2}{ }^{3}}{\alpha_{2}{ }^{2}}+\frac{x_{3}{ }^{3}}{\alpha_{3}{ }^{2}}=0
$$

wird von der Ebene des Knotens entweder in drei oder nux einer reellen Geraden getroffen, so lange der Knoten der allgemeinste, eiu $D_{6}$ ist. Hiernach sind zwei Arten zu unterscheiden. Den Uebergang vermitteln Flächen mit berührender Ebene des Knotens; die Discriminante der cubischen Form, welche durch die Knotengeraden gegeben ist, verschwindet für diese Art:

$$
\alpha_{1}+\alpha_{2}+\alpha_{3}=0
$$

und diese Gleichung stellt somit die Bedingung für das Auftreten eines $U_{7}$ dar.

Die Curve der Pentaederecken $C$ hat einen Rückkehrpunkt, der zur Hesse'schen Fläche gehörige Kegel $K$ berübrt die Ebene des hnotens längs der Rückkehrtangente von $C$.

Der Kegel $P$ ist jetzt darstellbar durch

$$
\begin{aligned}
\left(\alpha_{1} u_{1}+\alpha_{2} u_{2}+\alpha_{3} u_{3}\right)\left(\alpha_{1}\left(u_{1}^{2}+u_{2} u_{3}\right)\right. & +\alpha_{2}\left(u_{2}^{2}+u_{3} u_{1}\right) \\
& \left.+\alpha_{3}\left(u_{3}^{2}+u_{1} u_{2}\right)\right)=0,
\end{aligned}
$$

er ist demnach in einen Kegel zweiter Classe und eine auf ihm liegende Gerade (Classenscheitel einer beliebig wählbaren Leitlinie) zerfallen. Wie zu erwarteu war, ist letztere die Rückkebrtangente von $C$. Die gegenseitige Lage dieser Gebilde ist auf Tafel III, Fig. 2 dargestellt, in der der Wendepunkt von $C$ unendlich fern angenom-
men ist. 
Die Ebenen der zerfallenden Polaren zeigen insofern ein verschiedenes Verhalten, als die eine sich um die Rückkehrtangente dreht, die andere Tangentenebene des Kegels ist.

Alle übrigen Flächen sind Tetraederfï̈chen (vergl. $\$ 18$.), $C$ zerfâllt in Gerade, jetzt als Kanten des Tetraeders aufuufassen. In diese Arten gehen dann auch die Regelflächen ein. Die Ausartungen der Curve $C$ für die einzelnen hierher gehörigen Flächen des $\$ 18$. sind die folgenden:

Art:

C

4) und 5) mit $U_{6}$

8) mit $U_{8}$

Drei Gerade, von denen sich zwei im Knoten schneiden.

Regelflächen 6) und 7) In jeder Ebene der beiden als $V_{3}$ aufaufassenden Punkte der Doppeldirectrix eine solche Curve.

Regelfä̀che 9)

In der Ebene des als $U_{8}$ aufzufassenden Punktes ein dreifache Linie.

Die Flächen 4) und 5) verhelfen uns, in Verbindung mit der gefundenen Bedingung für eiren $U_{7}$, zu Criterien für die verschiedenen Arten mit $U_{6}$. Ausgehend von den genannten, welche einem unendlich grossen Werthe einer der Grössen * entsprechen, finden wir:

a) Bei der Annahme reeller $x_{1}, x_{2}, x_{3}$ in der Fläche

$$
x_{4}\left(x_{1}+x_{2}+x_{3}\right)^{2}+\frac{x_{2}{ }^{3}}{\alpha_{1}{ }^{2}}+\frac{x_{2}{ }^{3}}{\alpha_{2}{ }^{2}}+\frac{x_{3}{ }^{3}}{\alpha_{3}{ }^{2}}=0 \ldots \alpha_{1}<\alpha_{2}<\alpha_{3},
$$

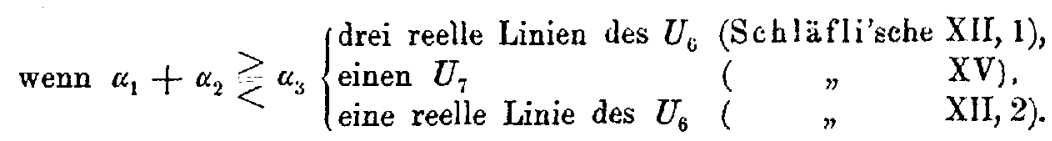

Ist $\alpha_{3}$ imaginär, so ist die Fläche ebenfalls von der letzten Art.

b) Bei der Annahme conjugirter $x_{1}$ und $x_{2}$ in der Fläche

$$
x_{4}\left(2 x_{1}+x_{3}\right)+\frac{\left(x_{1}+i x_{2}\right)^{3}}{\left(\alpha_{1}+i \alpha_{2}\right)^{2}}+\frac{\left(x_{1}-i x_{2}\right)^{3}}{\left(\alpha_{1}-i \alpha_{2}\right)^{2}}+\frac{x_{3}{ }^{3}}{\alpha_{3}^{2}}=0,
$$

wenn $2 \alpha_{1} \lesseqgtr \alpha_{3}\left\{\begin{array}{cc}\text { Schläfli'sche XII, 1, } \\ ", & \text { XV, } \\ " & \text { XII, 2. }\end{array}\right.$ 


\section{$\$ 21$. \\ Zusammenstellung der Resultate.}

Wir geben zum Schlusse eine Tabelle (Tafel IV), welche einerseits die möglichen Pentaeder einer Flächenart, andererseits alle Arten, welche einem und demselben Pentaeder zukommen, erkennen lässt. Das Vorhandensein einer Fläche ist durch ein + angedeutet. Die Schläfli'schen Bezeichnungen sind durch zwei Zahlen charakterisirt, so dass keine Verwechselung mit der von Herrn Klein und mír benutzten entstehen kann. Eingeklammerte Zahlen bei Flächen mit imaginären Knoten bezeichnen die Flächen mit reellen bez. ohne Knoten, zwischen denen die betreffenden sich vorfinden, ohne einen Uebergang zu einer andern Art zu bewirken*).

*) Ich beabsichtige im Anschlusse an diese Arbeit eine Collection von Modellen der versehiedenen Arten von Flächen dritter Ordnung und einiger ihrer Hesse'schen Flächen erscheinen zu lasser, welche das concrete Eriassen der Singularitiaten erleichtem werden.

In December 1877. 